\title{
Stereocontrolled Synthesis of $\alpha$-Xylofuranosides Using a Conformationally Restricted Donor
}

\author{
Li Zhang, Ke Shen, Hashem A. Taha, and Todd L. Lowary*(i)
}

Alberta Glycomics Centre and Department of Chemistry, University of Alberta, 11227 Saskatchewan Drive, Edmonton, Alberta T6G 2G2, Canada

\section{Supporting Information}

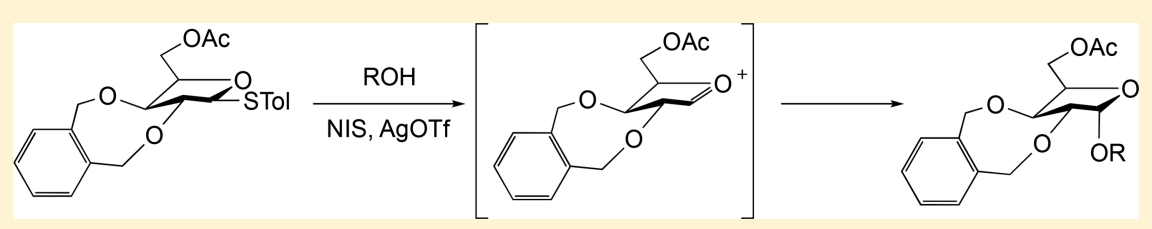

ABSTRACT: A number of biologically relevant glycoconjugates possess 1,2-cis-furanosidic linkages, a class of glycosidic bond that remains challenging to introduce with high stereoselectivity. In this paper, we report an approach to one family of such linkages, $\alpha$-xylofuranosides, via the use of thioglycoside donors possessing a conformationally restricting xylylene protecting group. The method was shown to provide the desired targets in good to excellent yield and stereoselectivity. Computational investigations support the proposal that the protecting group locks the electrophilic intermediate in these reactions into a conformation that leads to the high selectivity. The power of the methodology was demonstrated through the synthesis of a complex hexasaccharide motif from lipoarabinomannan, an immunomodulatory polysaccharide from mycobacteria.

\section{INTRODUCTION}

Glycoconjugates containing monosaccharide residues in the furanose ring form are present in a number of pathogenic organisms, including Aspergillus fumigatus and Mycobacterium tuberculosis, the causative agents of invasive aspergillosis and tuberculosis, respectively. ${ }^{1-4}$ Generally present in the protective cell walls of the organisms that produce them, these molecules play important roles in disease progression and the interaction of the pathogen with the host immune system. Access to structurally defined glycoconjugates incorporating furanose rings is therefore of interest to develop probes for understanding their biological roles and as a prelude to possible therapeutic, vaccine, or diagnostic development. ${ }^{1,2}$ Such species are most conveniently obtained by chemical synthesis, and over the past several years many methods for accessing furanose-containing glycans have been developed. ${ }^{5,6}$

As is the case for their pyranoside counterparts, the stereocontrolled synthesis of 1,2-cis furanosides (e.g., 1 and 2, respectively, Figure 1A) is more challenging than that of 1,2trans furanosides. Participating protecting groups at $\mathrm{O}-2$ cannot be employed, and the inherently flexible five-membered ring often further complicates stereocontrol by providing two energetically similar reaction pathways that produce mixtures of products. ${ }^{5}$ Consequently, the majority of robust methods for the stereocontrolled synthesis of 1,2-cis-furanosides employ conformationally locking groups resulting in a single energetically favored reaction pathway. ${ }^{5,6}$ Such methods have previously been applied to the synthesis of $\beta$-arabinofuranosides (3, Figure 1B), ${ }^{7-9} \beta$-fructofuranosides (4), ${ }^{10}$ and $\alpha$ galactofuranosides $(\mathbf{5})$. $^{11-1}$

In this context, we have previously reported the preparation of $\beta$-arabinofuranosides using the 2,3-O-xylylene-protected

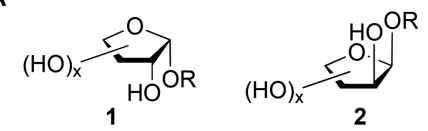

B

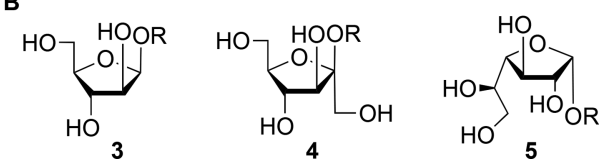

Figure 1. (A) Schematic representation of 1,2-cis- $\alpha$-furanosides (1) and 1,2-cis- $\beta$-furanosides (2). (B) Examples of $\beta$-arabinofuranosides (3), $\beta$-fructofuranosides (4), and $\alpha$-galactofuranosides (5).

arabinofuranosyl thioglycoside 6 (Figure $2 \mathrm{~A}$ ). ${ }^{14}$ This donor gave high 1,2-cis-selectivity in reactions with a range of acceptor molecules. The selectivity was proposed to arise from the cyclic xylylene protecting group fixing the electrophilic intermediate produced upon activation into a single conformationally rigid species. This donor is a particularly attractive reagent when there is a need to modify $0-5$ of the $\beta$ arabinofuranoside residue subsequent to glycosylation.

We describe here an extension of that work by exploring 2,3$O$-xylylene-protected xylofuranosyl thioglycosides (7 and 8) as agents for the stereocontrolled installation of $\alpha$-xylofuranoside bonds. We hypothesized that activation of 7 and 8 would lead to the formation of electrophilic intermediate (e.g., an oxacarbenium ion or a related ion pair) restricted in a conformation with $\mathrm{C}-3$ below the plane formed by the other

Received: February 12, 2018

Published: June 13, 2018 
A

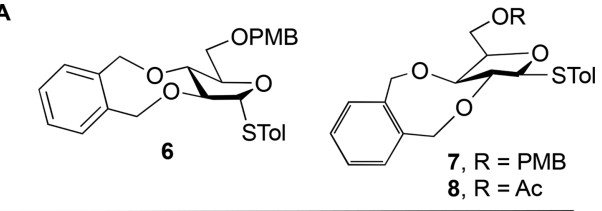

B

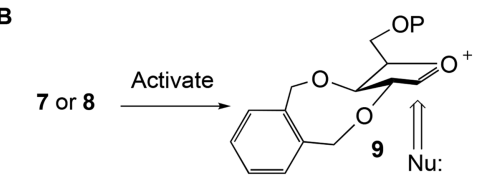

C

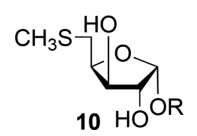

Figure 2. (A) 2,3-O-Xylylene-protected donor (6) for the stereoselective synthesis of $\beta$-arabinofuranosides and 2,3-O-xylyleneprotected donors (7 and 8) explored in this paper as reagents for synthesizing $\alpha$-xylofuranosides. (B) Central hypothesis of this work: that 7 and 8 will lead to an intermediate (e.g., 9) that preferentially reacts $^{15}$ with nucleophiles to produce $\alpha$-glycosides. (C) 5Thiomethyl- $\alpha$-xylofuranoside $(\alpha$-MTX) residue (10) present in mycobacterial LAM.

ring atoms (the $\mathrm{E}_{3}$ conformation). Such a species would be expected to react preferentially from the bottom face following the inside attack model ${ }^{15}$ of Woerpel, leading to the $\alpha$ glycoside (Figure 2B). Interest in synthesizing $\alpha$-xylofuranosides arises, in part, from the presence of 5-thiomethyl- $\alpha$ xylofuranoside ( $\alpha$-MTX) residues (e.g., 10, Figure 2C) in the polysaccharide lipoarabinomannan (LAM), a key immunomodulatory polysaccharide in the cell wall of mycobacteria. ${ }^{16}$ Among the organisms possessing this motif is M. tuberculosis. The preparation of $\alpha$-MTX-containing glycoconjugates is of interest to provide tools for studying the biological role of this unusual monosaccharide. ${ }^{17} \mathrm{We},{ }^{18,19}$ and others, ${ }^{16,20,21}$ have previously prepared oligosaccharides containing $\alpha$-MTX residues, but the xylofuranosylation reactions often proceeded with modest stereoselectivity, and frequently the mixtures were difficult to purify.

\section{RESULTS AND DISCUSSION}

Preparation of 2,3-O-Xylylene-Protected Xylofuranoside Thioglycoside Donors. The key intermediate in the synthesis of 7 and $\mathbf{8}$ (Scheme 1) is the previously reported trityl derivative 11, which can be conveniently prepared in four steps from D-xylose. ${ }^{18,22}$ Reaction of 11 with $\alpha, \alpha^{\prime}$-dibromo-oxylene and $\mathrm{NaH}$ in DMF and subsequent removal of the trityl group under acidic conditions gave alcohol 12 in $41 \%$ yield over the two steps. The major byproduct was a dimer resulting from two equivalents of the 11 reacting with the alkylating agent. A $p$-methoxybenzyl (PMB) group was introduced on $\mathrm{O}$ 5 to obtain building block 7 in $92 \%$ yield upon reaction of $\mathbf{1 2}$ with sodium hydride and $\mathrm{PMBCl}$. Alternatively, acetylation of 12 with acetic anhydride and pyridine gave $\mathbf{8}$ in quantitative yield.

Optimization of Glycosylation Conditions. With donors $\mathbf{7}$ and $\mathbf{8}$ in hand, we first explored their reaction with a model acceptor, 1,2:3,4-di-O-isopropylidene- $\alpha$-D-galactopyranose (13), as detailed in Table 1 . The stereochemistry of the newly introduced glycosidic linkage could be identified by the coupling constant between $\mathrm{H}-1$ and $\mathrm{H}-2\left({ }^{3} J\right)$ in the ${ }^{1} \mathrm{H}$ NMR
Scheme 1. Synthesis of 7 and 8
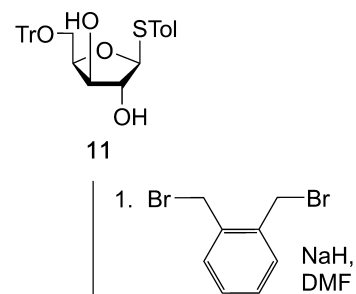

2. $p-\mathrm{TsOH}, \mathrm{CH}_{2} \mathrm{Cl}_{2}, \mathrm{CH}_{3} \mathrm{OH}$

$41 \%$ over two steps
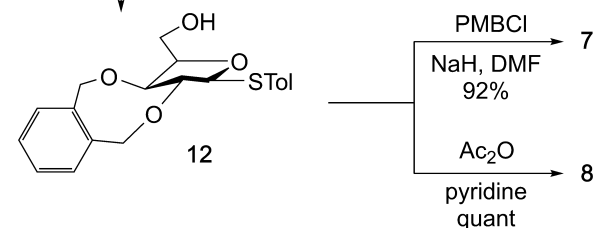

spectrum of the product. For the 1,2-cis $(\alpha)$ isomer, ${ }^{3} \mathrm{~J}$ is $\sim 5.0$ Hz. In contrast, ${ }^{3} \mathrm{~J}$ is $\sim 2.5 \mathrm{~Hz}$ for the 1,2 -trans $(\beta)$ isomer. ${ }^{14}$ Further support for these assignments came from the ${ }^{1} \mathrm{H}$ and ${ }^{13} \mathrm{C}$ NMR spectra of deprotected compounds, including a hexasaccharide target synthesized (see below), using this methodology. Data obtained from these deprotected compounds was consistent ${ }^{23}$ with the assignments made using the trends outlined above.

The effect of the solvent on the stereoselectivity of the reaction was investigated first. When 7 ( 1.3 equiv) was reacted with 13 (1.0 equiv) in the presence of $N$-iodosuccinimide (NIS) and silver triflate (AgOTf) in different solvents (Table 1 , entries 1-5), disaccharide 14 was obtained with $\alpha: \beta$ selectivities ranging from $\sim 1: 3$ to $\sim 2: 1$. The use of diethyl ether (entry 4) or a 1:3 mixture of toluene and dioxane (entry 5) provided the best $\alpha$-selectivity. These results are consistent with previous work indicating that that ethereal solvents tend to increase $\alpha$-stereoselectivity in glycosylations. ${ }^{24}$ It should be noted, however, that this effect in furanosides has not been widely studied.

The effects of the O-5 protecting group in the donor were next explored. When donor 7, which bears an electron-rich $p$ methoxybenzyl group at O-5, was reacted with 13 (entry 4), disaccharide 14 was generated in good yield and with moderate $\alpha$-selectivity $(\alpha: \beta=2.3: 1)$. The use of 8 , with an electron-withdrawing acetyl group at $\mathrm{O}-5$, gave the desired disaccharide $\mathbf{1 5}$ in good yield and with very good $\alpha$-selectivity $(\alpha: \beta=9.5: 1$, entry 6). Interestingly, this trend is opposite to what was observed with donor $\mathbf{6}$, which was used for the synthesis of $\beta$-arabinofuranosides. ${ }^{14}$ With $\mathbf{6}$, electron-rich groups on $\mathrm{O}-5$ led to higher 1,2-cis-selectivity. We do not understand why these differences are observed. Indeed, if the reaction proceeds through an oxacarbenium ion intermediate (see below), a more electron-rich donor should, in theory, be more selective.

Finally, glycosylations in diethyl ether with different equivalents of 8 were examined (entries 7 and 8). When 1.7 equiv of $\mathbf{8}$ was used, the desired disaccharide $\mathbf{1 5}$ was formed in the best yield (78\%) compared to when either 1.3 or 2.5 equiv was used. The $\alpha$-selectivity $(\alpha: \beta=9.5: 1)$ was the same in all cases.

In other optimization studies, the reaction concentration was shown to have no substantial effect on the yield and selectivity of glycosylations using $\mathbf{8}$ as the donor (Table S1). In addition, 
Table 1. Optimization of Glycosylation Conditions Using 7 and 8

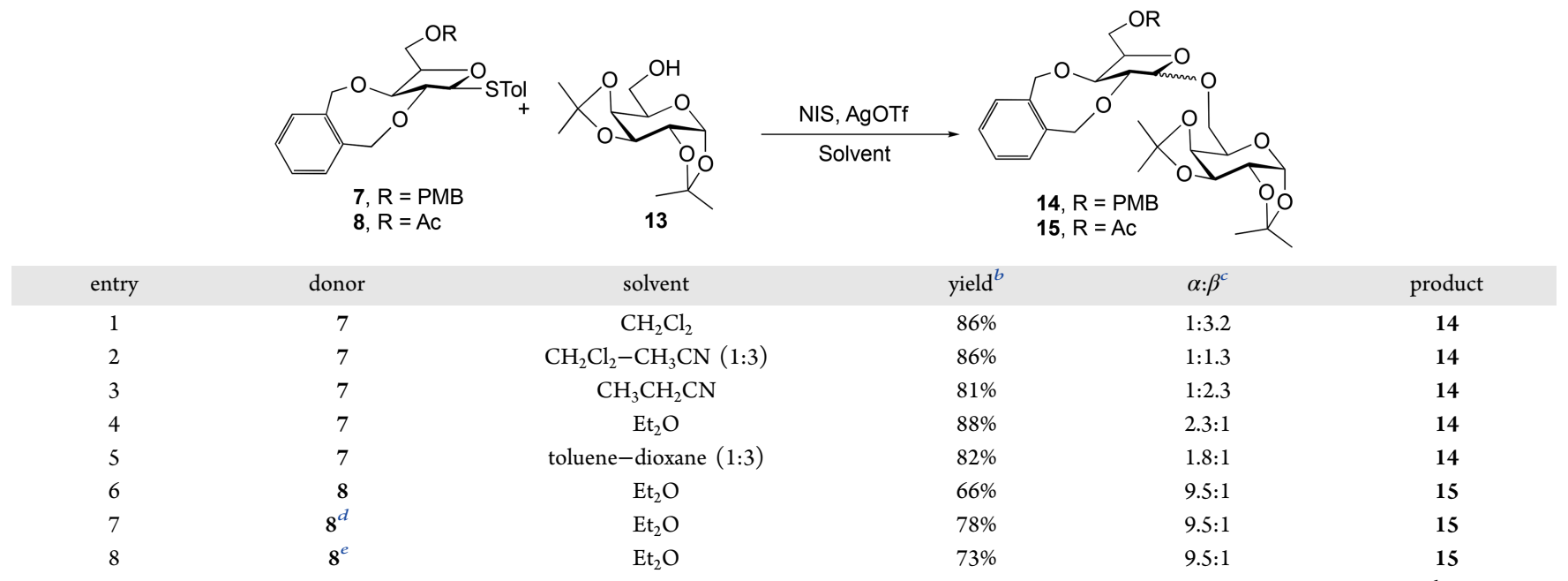

${ }^{a}$ Unless otherwise indicated, all reactions employed 1.3 equiv of donor, 1.0 equiv of $13,2.5$ equiv of NIS, and 0.25 equiv of AgOTf. ${ }^{b}$ Combined yield of $\alpha$ - and $\beta$-isomers. ${ }^{c}$ Determined from the ratio of $\mathrm{H}-1$ resonances in the ${ }^{1} \mathrm{H}$ NMR spectrum of the corresponding isomer mixture. ${ }^{d} 1.7$ equiv of donor used. ${ }^{e} 2.5$ equiv of donor used.

the $\beta$-anomer of $\mathbf{8}$ was prepared and shown to give glycoside products in yields and selectivity identical to 8 . The studies outlined above identified optimized conditions for these glycosylations: reaction of 1.7 equiv of donor 8 and 1.0 equiv of a glycosyl acceptor in the presence of 2.5 equiv of NIS and 0.25 equiv of AgOTf in diethyl ether at room temperature. Under these conditions, the desired glycoside was formed in high yield and with very good $\alpha$-selectivity.

Substrate Scope of the Xylofuranosylation. Having optimized the reaction, its use with a range of carbohydrate acceptors was investigated. Glycosylations with mono- and disaccharides 16-23 proceeded smoothly, giving products 25-32 (Table 2, entries 2-9) in good to excellent yield (67$96 \%)$ and $\alpha$-stereoselectivity $(\alpha: \beta 7: 1$ to $>20: 1)$. The results were not uniformly excellent, however. Glycosylation of $\mathbf{2 4}$ with 8 (entry 10) provided disaccharide 33 with essentially no stereoselectivity $(\alpha: \beta$ 1.2:1). In contrast, glycosylation of mannopyranosides $\mathbf{2 0}$ and 23 , which contain a free C-4 hydroxyl group, afforded disaccharides 29 and 32 in excellent yield and $\alpha$-stereoselectivity $(\alpha: \beta>17.7: 1)$. This is significant, as the $\alpha$-D-xylofuranosyl-( $1 \rightarrow 4)-\alpha$-D-mannopyranose linkage produced in these reactions is the same core structure as that present in $\alpha$-MTX-functionalized mycobacterial LAM. It should be noted that another advantage of the xylylene protecting group is that when mixtures are produced, typically the two products are can be separated without difficulty. For example, the separation of the two diastereomers of 33 could be easily achieved.

Computational Investigations of the Reaction. In an effort to understand the selectivity obtained in the reaction, we carried out quantum mechanical calculations to determine the energetics of the reaction coordinate. First, the optimized geometry of the oxacarbenium ion that results from activation of the thioglycoside was determined at the HF/6-311G(d,p)// B3LYP/6-31G(d) level of theory. Although the true intermediate in these reactions is likely not a "naked" oxacarbenium ion, ${ }^{25}$ this intermediate was chosen to simplify the calculations. In addition, a model with a 5-O-benzoyl group (34, Figure 3) was used to facilitate subsequent transition state calculations to avoid undesired bond stretch/bend frequencies associated with the methyl group of the acetate ester. As we hypothesized at the outset of this project (Figure 2B), the lowest energy conformation of $\mathbf{3 4}$ is the $\mathrm{E}_{3}$ envelope (Figure 3, middle). This is similar in structure to the lowest energy conformation of a xylo-configured 2,3,5-tri-O-methylated oxacarbenium ion (35), calculated earlier by Codee and coworkers. $^{26}$ Oxacarbenium ion 35 adopts a ${ }^{4} \mathrm{~T}_{3}$ twist conformation, with $\mathrm{C}-4$ above and $\mathrm{C}-3$ below the plane formed by $\mathrm{C}-1, \mathrm{C}-2$, and the ring oxygen.

From the oxacarbenium ion, two pathways were examined: one where the nucleophile (methanol was used in these calculations) can attack from the top face resulting in 1,2-trans product or from the bottom face resulting in a 1,2-cis product. Both products were optimized, and their geometries are shown in Figure 4. To simplify the calculations, we assumed that loss of the proton from the methanol was fast and occurred following glycosylation (i.e., after $\mathrm{C}-\mathrm{O}$ bond formation). As such the optimized products are protonated glycosides. The Supporting Information contains animations showing the both approaches of the nucleophile to 34. The 1,2-cis product adopts the same conformation as the oxacarbenium ion $\left(\mathrm{E}_{3}\right)$ whereas the 1,2-trans-product has a slightly different conformation $\left({ }^{4} \mathrm{~T}_{3}\right)$. These are the same as the tri-Omethylated derivatives studied previously by Codée and coworkers. ${ }^{26}$ The relative energies that were observed indicated that the 1,2 -cis product is $6.3 \mathrm{kcal} / \mathrm{mol}$ lower in energy than the 1,2-trans product.

Using the optimized geometries of the two products, we carried out coordinate scans on the $\mathrm{C} 1-\mathrm{O} 1$ bond at the HF/6$31 \mathrm{G}(\mathrm{d})$ level to obtain a picture of the energetics of the reaction coordinate. As can be observed in Figure 5, the path toward the 1,2-cis product has a lower energy barrier than that producing the 1,2-trans-product. These data suggest that nucleophilic trapping of $\mathbf{3 4}$ by a nucleophile from the bottom face of the ring is kinetically favored. Each of the scan structures was used as input for transition state calculations, which were done using HF/6-31G(d) level of theory. Frequency analysis showed that one imaginary frequency was obtained for each of the transition state structures. Geometries earlier in the reaction coordinate showed imaginary frequencies of -147.9 and $-155.2 \mathrm{~cm}^{-1}$ for the cis and trans products, respectively. These correspond to $\mathrm{OCH}_{3}$ rotational 
Table 2. Xylofuranosylation of Various Carbohydrate Acceptors by $8^{a}$

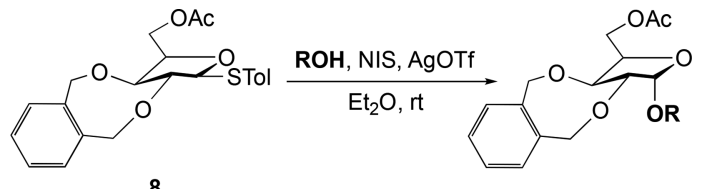

8

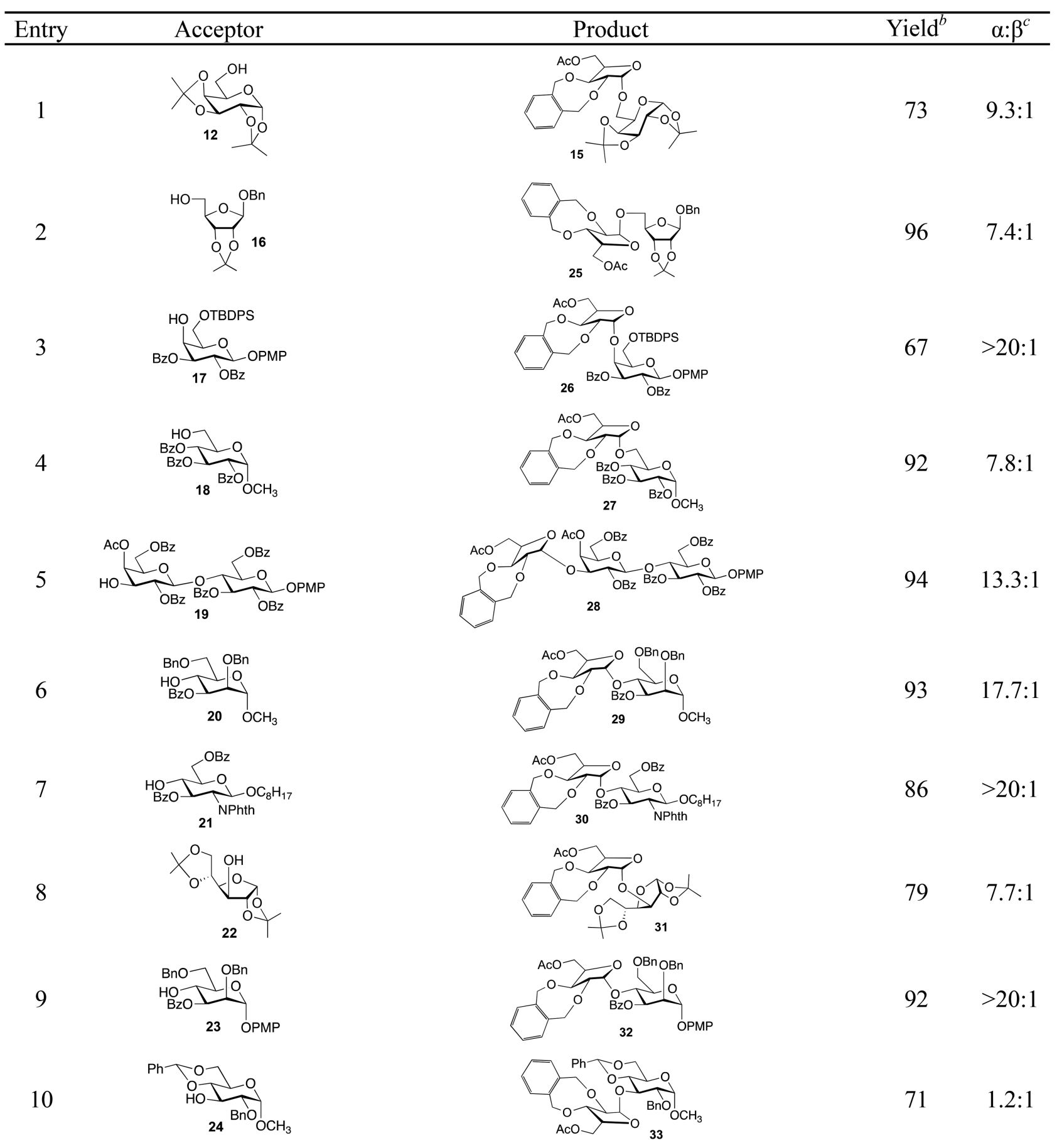

${ }^{a}$ Reaction conditions: 1.7 equiv of donor, 1 equiv of acceptor, 2.5 equiv of NIS, and 0.25 equiv of AgOTf. ${ }^{b}$ Combined yield of $\alpha$ - and $\beta$-isomers. ${ }^{c}$ Determined from the ratio of $\mathrm{H}-1$ resonances in the ${ }^{1} \mathrm{H}$ NMR spectrum of the corresponding isomer mixture.

vibrations. Higher energy structures near the transition state showed imaginary frequencies of -186.9 and $-233.9 \mathrm{~cm}^{-1}$ corresponding to the desired $\mathrm{C} 1-\mathrm{O} 1$ bond stretching.

Taken together, these computational results provide support for our hypothesis that electrophilic intermediates derived from 2,3-O-xylylene-protected-xylofuranoside glycosyl donors adopt conformations that should, based on Woerpel's inside attack model, ${ }^{15}$ lead to $\alpha$-glycosides. Moreover, the transition state calculations suggest that trapping of these electrophiles by nucleophiles from the bottom face, producing $\alpha$-glycosides, is 

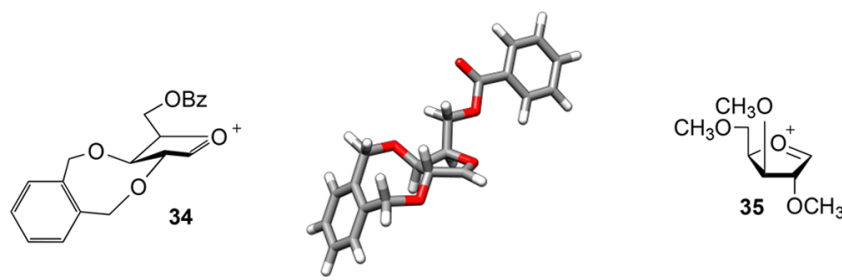

Figure 3. Structure of oxacarbenium ion 34 (left), its low-energy conformation as determined by DFT calculations (middle), and a 2,3,5-tri-O-methyl-xylofuranosyl oxacarbenium (35) studied earlier by Codée and co-workers (right). ${ }^{26}$
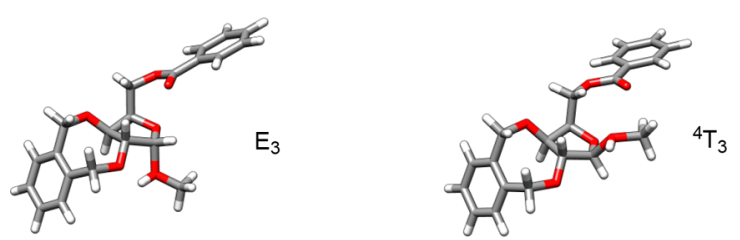

Figure 4. Optimized geometries of the 1,2-cis (left) and 1,2-trans (right) glycoside products formed upon trapping of oxcarbenium ion 34 with methanol.

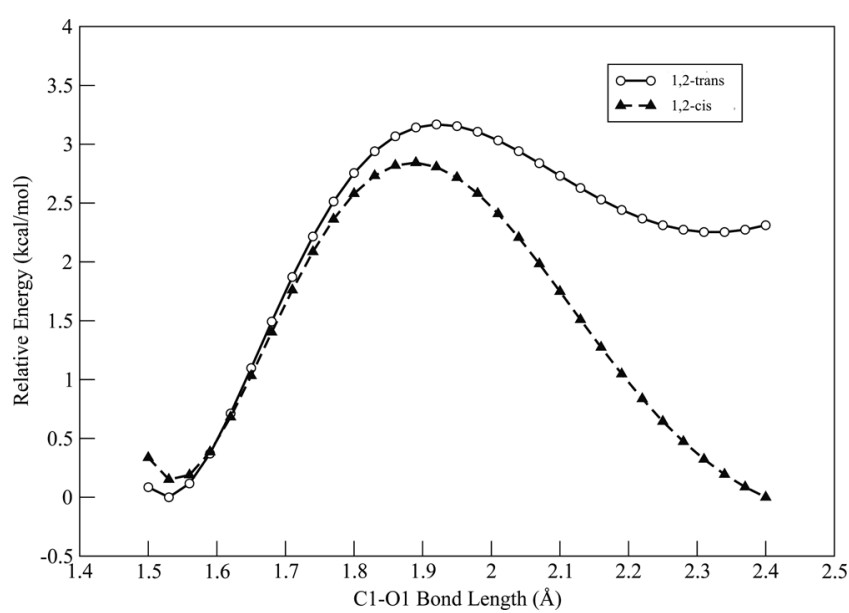

Figure 5. Coordinate scan of the $\mathrm{C} 1-\mathrm{O} 1$ bond from 1.5 to $2.4 \AA$ in the 1,2-trans (open circles) and 1,2-cis (filled triangles) methyl glycoside products arising from trapping of 34 with methanol.

indeed energetically favored. Although a similar hypothesis was proposed for reactions leading to $\beta$-arabinofuranosides from $\mathbf{6}^{14}$ the calculations here represent the first support for the origin of the stereoselectivity in glycosylation reactions with xylylene-protected-furanoside donors. It should be noted that remote participation by the $\mathrm{O}-5$ ester group could also provide an explanation for the high $\alpha$-selectivity. We think this unlikely as the conformational rigidity imposed on the furanose ring by the xylylene group would result in significant torsional strain should remote participation occur. In addition, the use of other electron-withdrawing, but not participating, groups at this position (see below) also give high yields of the 1,2-cisproduct.

Synthesis of an $\alpha$-MTX-Containing Hexasaccharide Fragment of LAM. The studies described above demonstrate the successful use of 2,3-O-xylylene-protected thioglycoside 8 for the stereoselective synthesis of $\alpha$-xylofuranosides and, in particular, the $\alpha$-D-xylofuranosyl-(1 $1 \rightarrow 4)-\alpha$-D-mannopyranose motif. We then turned our attention to applying the methodology to a more complex biologically relevant molecule. We selected as a target a hexasaccharide fragment of mycobacterial LAM (36, Scheme 2). This molecule, and related $\alpha$-MTX-containing compounds, has recently been incorporated into a glycan array for use in mapping the specificity of proteins that recognize mycobacterial glycans. ${ }^{19}$

The previous synthesis of 36 did not employ 2,3-O-xylyleneprotected donors to introduce either the $\alpha$-MTX or the $\beta$ arabinofuranoside residues. ${ }^{19}$ Therefore, we endeavored to use both $\mathbf{6}$ and a derivative of 8 for their installation. To streamline the synthesis, we explored the use of the 5-O-mesyl-2,3-Oxylylene-thioglycoside 37 in place of 8 . We postulated that the electron-withdrawing nature of the mesyl group would lead to high $\alpha$-selectivity, and the resulting product could then be subjected directly to a substitution reaction to introduce the thiomethyl group. This approach would save two steps (deacetylation and mesylation) postglycosylation compared to if $\mathbf{8}$ were used. We also planned to use thioglycoside $\mathbf{3 8}$ and the previously reported trisaccharide $39^{19}$ in the synthesis of 36. The synthesis of $\mathbf{3 7}$ and $\mathbf{3 8}$ is shown in Scheme 3 and was achieved from 12 (above) and the known thioglycoside 40, ${ }^{27}$ respectively.

Implementation of this strategy is shown in Scheme 4. The synthesis began with the treatment of trisaccharide 39 and thioglycoside 6 with NIS and trimethylsilyl trifluoromethanesulfonate (TMSOTf) leading to the formation of tetrasaccharide 42 in $80 \%$ yield. The PMB group was then cleaved by treatment with TFA yielding a 91\% yield of alcohol 43 . Glycosylation of 43 with 38 , again promoted by NIS and TMSOTf, resulted in an $82 \%$ yield of pentasaccharide 44 , which, upon treatment with hydrazine acetate, gave 45, the substrate for the key xylofuranosylation reaction. We were pleased to discover that the reaction of $\mathbf{4 5}$ with 37 did indeed give the desired product, 46 , in an excellent $87 \%$ yield. None of

Scheme 2. Retrosynthetic Analysis of 36
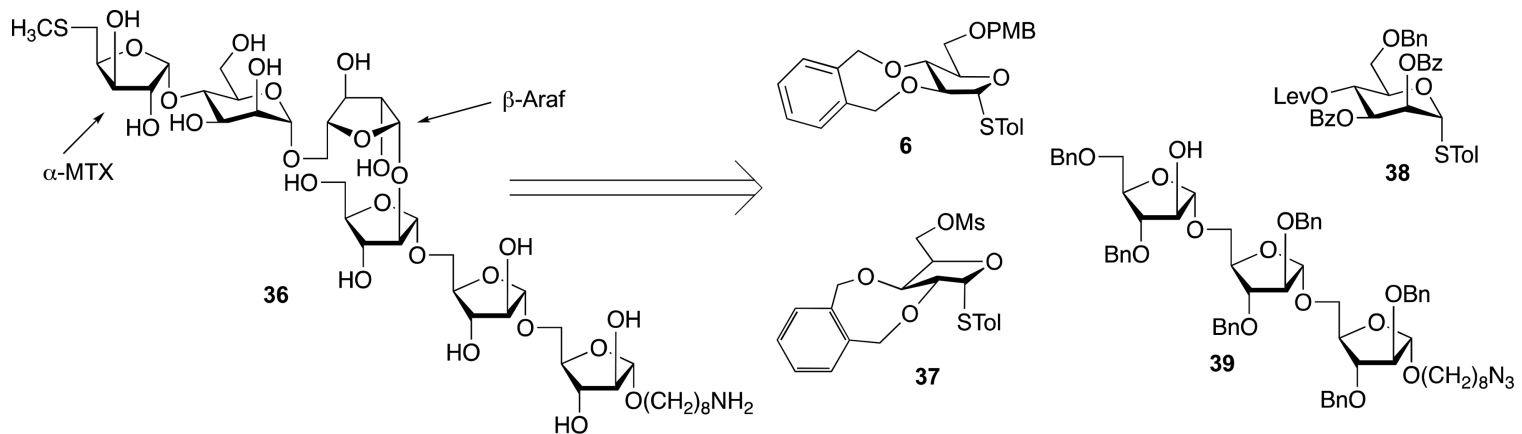
Scheme 3. Synthesis of Thioglycosides 37 (A) and 38 (B)

A
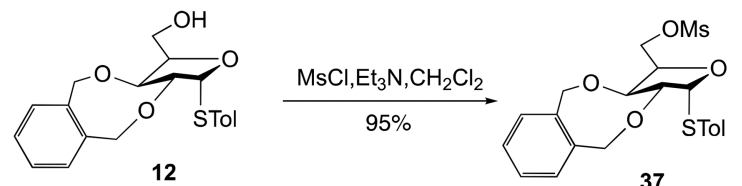

37

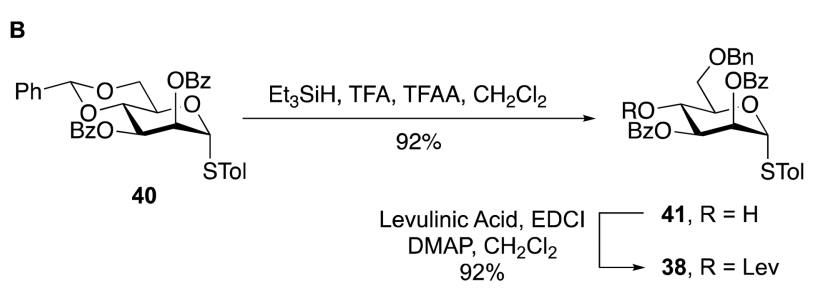

the $\beta$-glycoside product was detected in the reaction. It should be noted, however, that this reaction did require optimization. In particular, donor $\mathbf{3 7}$ was not soluble in diethyl ether, the solvent shown (Table 1) to be optimal for the high degree of $\alpha$-selectivity in these reactions. Fortunately, the use of THF, in which 37 is soluble, provided the desired $\alpha$-glycoside with high selectivity. The high yield obtained in formation of $\mathbf{4 6}$ suggests that the origin of the $\alpha$-selectivity using 8 arises from the electron-withdrawing nature of the O-5 substituent, not remote participation. If participation were essential, the reaction of 45 with 37 , which has a nonparticipating mesylate at $\mathrm{O}-5$, would be expected to give the product with lower 1,2cis selectivity.

Having installed all of the monosaccharide residues, the mesyl group was displaced, and the benzoate esters cleaved, using an excess of sodium thiomethoxide in acetonitrile at reflux. The conversion of 46 into 47 proceeded in $74 \%$ yield.
Finally, the benzyl and xylylene protecting groups were cleaved using dissolving metal reduction, leading to a $74 \%$ yield of hexasaccharide 36 . The synthesis of the mycobacterial LAM fragment thus could be achieved in seven steps and $26 \%$ overall yield from 39. This compares favorably to our previous synthesis of this compound, also from $39,{ }^{19}$ which required 11 steps and two difficult chromatographic purifications, leading to the formation of the product in $12 \%$ overall yield.

\section{SUMMARY}

In conclusion, a novel stereoselective 1,2-cis $\alpha$-xylofuranosylation employing a conformationally constrained 2,3-O-xylyleneprotected xylofuranosyl donor has been developed. Optimization of the glycosylation conditions showed that treatment of 1.7 equiv of $p$-tolyl 5-O-acetyl-1-thio-2,3-O-xylylene- $\alpha$-Dxylofuranoside (8) with 1.0 equiv of various acceptors, in the presence of 2.5 equiv of NIS and 0.25 equiv of AgOTf in diethyl ether at room temperature, gives the product in high yield and in high $\alpha$-selectivity. Computational investigations suggest that the reaction produces an electrophilic intermediate with a conformation that favors the formation of the desired $\alpha$-glycoside. In an application of the method, we prepared a hexasaccharide fragment of mycobacterial LAM in which two 1,2-cis-furanosyl residues, a $\beta$-arabinofuranoside and an $\alpha$-xylofuranoside, were installed using 2,3-O-xylyleneprotected thioglycoside donors. This synthesis highlights the power of these reagents in the assembly of glycans containing 1,2-cis-furanosides, particularly those with modifications at $\mathrm{O}$ 5.

\section{EXPERIMENTAL SECTION}

General Methods. All reagents used were purchased from commercial sources and were used without further purification unless

\section{Scheme 4. Synthesis of Hexasaccharide 36}
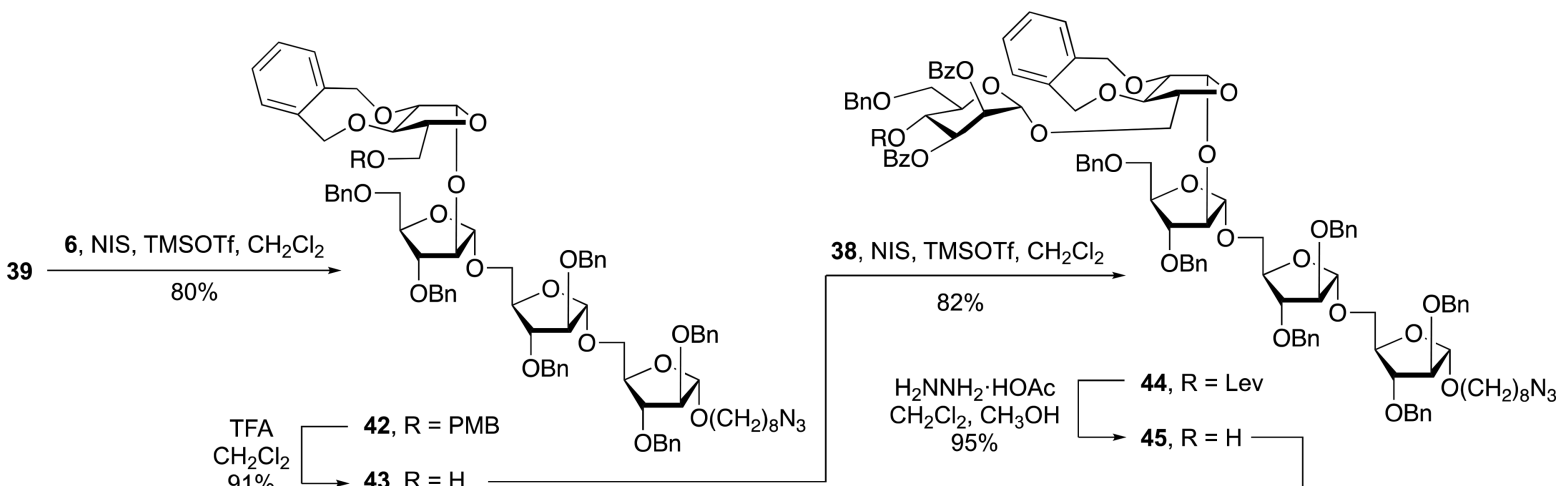

$91 \%$

$43, R=H$
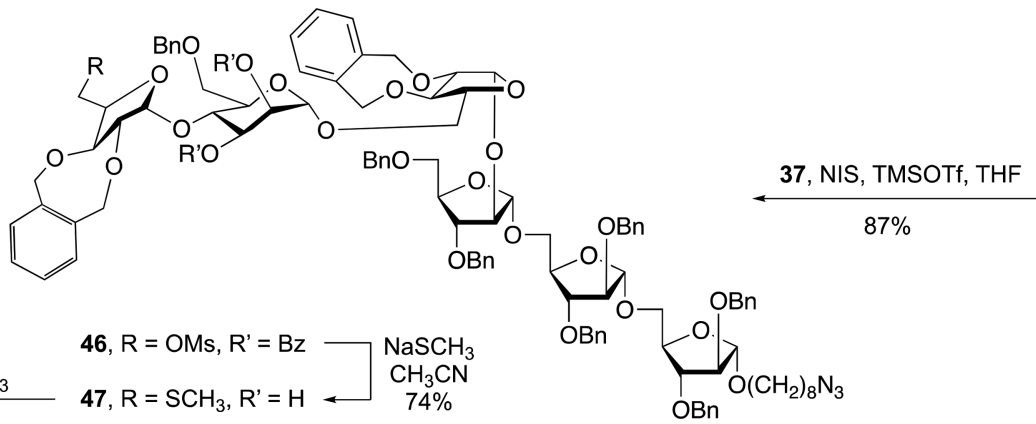

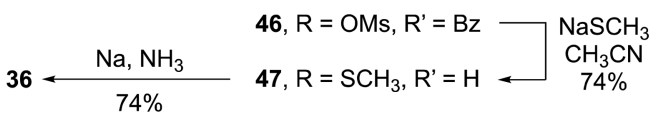


noted. Solvents used in reactions were purified by successive passage through columns of alumina and copper under nitrogen. Unless stated otherwise, all reactions were carried out under a positive pressure of argon. Reactions were monitored by TLC on Silica Gel $60-\mathrm{F}_{254}(0.25$ $\mathrm{mm})$, and spots were visualized under UV light $(254 \mathrm{~nm})$ and/or stained by charring with acidified anisaldehyde solution in ethanol. Column chromatography was performed on Silica Gel 60 (40-60 $\mu \mathrm{m})$ or $\mathrm{C}_{18}$ silica gel (35-70 $\mu \mathrm{m}$, Toronto Research Chemicals). ${ }^{1} \mathrm{H}$ NMR spectra were recorded at 500,600 , or $700 \mathrm{MHz}$, and chemical shifts are referenced to $\mathrm{CHCl}_{3}\left(7.26, \mathrm{CDCl}_{3}\right)$ or $\mathrm{CH}_{3} \mathrm{OD}$ (3.35, $\left.\mathrm{CD}_{3} \mathrm{OD}\right) .{ }^{13} \mathrm{C}$ NMR spectra were recorded at 125 or $175 \mathrm{MHz}$, and ${ }^{13} \mathrm{C}$ chemical shifts are referenced to $\mathrm{CDCl}_{3}\left(77.06, \mathrm{CDCl}_{3}\right)$ or $\mathrm{CD}_{3} \mathrm{OD}\left(49.0, \mathrm{CD}_{3} \mathrm{OD}\right)$. Assignments of NMR spectra were made on the basis of two-dimensional experiments $\left({ }^{1} \mathrm{H}-{ }^{1} \mathrm{H}\right.$ COSY, HSQC, and HMBC). ESI-MS spectra were recorded on samples suspended in THF or $\mathrm{CH}_{3} \mathrm{OH}$ with added $\mathrm{NaCl}$. Optical rotations were measured at $22 \pm 2{ }^{\circ} \mathrm{C}$ at the sodium D-line $(589 \mathrm{~nm})$ and are in units of deg. $\mathrm{mL}(\mathrm{dm} \cdot \mathrm{g})^{-1}$.

General Procedure for Glycosylation Reactions. To a mixture of donor $(1.7 \mathrm{mmol})$ and acceptor $(1 \mathrm{mmol})$ in $\mathrm{Et}_{2} \mathrm{O}$ were added $4 \AA$ molecular sieves $(200 \mathrm{mg})$ at rt. After stirring for $1 \mathrm{~h}$, NIS $(2.5 \mathrm{mmol})$ and $\operatorname{AgOTf}(0.25 \mathrm{mmol})$ were added to the mixture. The reaction was monitored by TLC, and after stirring for $2 \mathrm{~h}$ at $\mathrm{rt}$, the reaction was quenched by the addition of $\mathrm{Et}_{3} \mathrm{~N}$. The solution was diluted with $\mathrm{CH}_{2} \mathrm{Cl}_{2}$ and filtered through Celite. The filtrate was then washed with satd aq. $\mathrm{Na}_{2} \mathrm{~S}_{2} \mathrm{O}_{3}$ and brine. The organic layer was subsequently dried with anhydrous $\mathrm{Na}_{2} \mathrm{SO}_{4}$, filtered, and concentrated, and the residue was purified by flash silica gel column chromatography (hexaneEtOAc) to give the product.

p-Tolyl 5-O-p-Methoxybenzyl-1-thio-2,3-O-xylylene- $\beta$-D-xylofuranoside (7). To a solution of $12(358 \mathrm{mg}, 1.00 \mathrm{mmol})$ in DMF $(6.0$ $\mathrm{mL})$ at $0{ }^{\circ} \mathrm{C}$ were added $\mathrm{NaH}(60 \mathrm{mg}, 1.50 \mathrm{mmol})$ and $\mathrm{PMBCl}(173$ $\mu \mathrm{L}, 1.50 \mathrm{mmol}$ ). The solution was stirred at $\mathrm{rt}$ for $2 \mathrm{~h}$, and the reaction was then quenched by the addition of satd aq. $\mathrm{NH}_{4} \mathrm{Cl}$. Dilution of the mixture with $\mathrm{CH}_{2} \mathrm{Cl}_{2}$ provided a solution that was then washed with brine. The organic layer was subsequently dried with anhydrous $\mathrm{Na}_{2} \mathrm{SO}_{4}$, filtered, and concentrated, and the residue was purified by silica gel column chromatography (5:1, hexanesEtOAc) to give $7(439 \mathrm{mg}, 92 \%)$ as a white solid. $R_{\mathrm{f}} 0.5$ (3:1, hexanes-EtOAc); $[\alpha]_{\mathrm{D}}=-56.8\left(c=0.8, \mathrm{CHCl}_{3}\right) ;{ }^{1} \mathrm{H}$ NMR $(500$ $\left.\mathrm{MHz}, \mathrm{CDCl}_{3}\right) \delta_{\mathrm{H}} 7.43-6.86(\mathrm{~m}, 12 \mathrm{H}, \mathrm{ArH}), 5.21(\mathrm{~d}, 1 \mathrm{H}, J=5.1 \mathrm{~Hz}$, $\mathrm{H}-1), 4.81\left(\mathrm{~d}, 1 \mathrm{H}, J=11.6 \mathrm{~Hz}, \mathrm{OCH}_{2} \mathrm{Ph}\right), 4.69(\mathrm{~d}, 1 \mathrm{H}, J=11.5 \mathrm{~Hz}$, $\left.\mathrm{OCH}_{2} \mathrm{Ph}\right), 4.61\left(\mathrm{~d}, 1 \mathrm{H}, J=11.5 \mathrm{~Hz}, \mathrm{OCH}_{2} \mathrm{Ph}\right), 4.56(\mathrm{~d}, 1 \mathrm{H}, J=11.6$ $\left.\mathrm{Hz}, \mathrm{OCH}_{2} \mathrm{Ph}\right), 4.51\left(\mathrm{~d}, 1 \mathrm{H}, J=11.6 \mathrm{~Hz}, \mathrm{OCH}_{2} \mathrm{Ph}\right), 4.43(\mathrm{~d}, 1 \mathrm{H}, J=$ $\left.11.6 \mathrm{~Hz}, \mathrm{OCH}_{2} \mathrm{Ph}\right), 4.41(\mathrm{q}, 1 \mathrm{H}, J=5.8 \mathrm{~Hz}, \mathrm{H}-4), 4.12(\mathrm{dd}, 1 \mathrm{H}, J=$ 3.6, $5.8 \mathrm{~Hz}, \mathrm{H}-3), 4.08(\mathrm{dd}, 1 \mathrm{H}, J=5.1,3.6 \mathrm{~Hz}, \mathrm{H}-2), 3.81-3.78(\mathrm{~m}$, $4 \mathrm{H}, \mathrm{H}-5^{\prime}, \mathrm{OCH}_{3}$ ), 3.74 (dd, $\left.1 \mathrm{H}, J=10.3,6.2 \mathrm{~Hz}, \mathrm{H}-5\right), 2.28(\mathrm{~s}, 3 \mathrm{H}$, $\left.\mathrm{ArCH}_{3}\right) ;{ }^{13} \mathrm{C}$ NMR $\left(125 \mathrm{MHz}, \mathrm{CDCl}_{3}\right) \delta_{\mathrm{C}} 159.3(\mathrm{Ar}), 137.6(\mathrm{Ar})$, 135.6 (Ar), 132.3 (2C, Ar), 130.5 (Ar), 130.4 (Ar), 129.7 (2C, Ar), 129.5 (Ar), 129.5 (2C, Ar), 129.2 (Ar), 128.8 (Ar), 128.4 (Ar), 128.1 (Ar), 113.8 (2C, Ar), 89.6 (C-1), 87.8 (C-2), 80.1 (C-3), 77.3 (C-4), $73.2\left(\mathrm{OCH}_{2} \mathrm{Ph}\right), 70.7\left(\mathrm{OCH}_{2} \mathrm{Ph}\right), 70.0\left(\mathrm{OCH}_{2} \mathrm{Ph}\right), 68.5(\mathrm{C}-5), 55.5$ $\left(\mathrm{OCH}_{3}\right), 21.2\left(\mathrm{ArCH}_{3}\right)$; HRMS-ESI $m / z[\mathrm{M}+\mathrm{Na}]^{+}$calcd for $\mathrm{C}_{28} \mathrm{H}_{30} \mathrm{NaO}_{5} \mathrm{~S}$ : 501.1706. Found: 501.1699.

p-Tolyl 5-O-Acetyl-1-thio-2,3-O-xylylene- $\beta$-D-xylofuranoside (8). To a solution of $12(358 \mathrm{mg}, 1.0 \mathrm{mmol})$ in pyridine $(5.0 \mathrm{~mL})$ was added acetic anhydride $(5.0 \mathrm{~mL})$ at $\mathrm{rt}$. The solution was stirred at $\mathrm{rt}$ for $2 \mathrm{~h}$, and then the reaction was quenched by the addition of $\mathrm{CH}_{3} \mathrm{OH}$, followed by coevaporation with toluene to remove the pyridine. The residue was purified by silica gel column chromatography $(6: 1$, hexanes-EtOAc) to afford $8(381 \mathrm{mg}, 95 \%)$ as a colorless oil. $R_{\mathrm{f}} 0.3\left(4: 1\right.$, hexanes-EtOAc); $[\alpha]_{\mathrm{D}}=-132.2\left(c=1.2, \mathrm{CHCl}_{3}\right)$; ${ }^{1} \mathrm{H}$ NMR $\left(500 \mathrm{MHz}, \mathrm{CDCl}_{3}\right) \delta_{\mathrm{H}} 7.42-7.29(\mathrm{~m}, 6 \mathrm{H}, \mathrm{ArH}), 7.09-7.04$ $(\mathrm{m}, 2 \mathrm{H}, \mathrm{ArH}), 5.01(\mathrm{~d}, 1 \mathrm{H}, J=7.5 \mathrm{~Hz}, \mathrm{H}-1), 4.96(\mathrm{~d}, 1 \mathrm{H}, J=12.7$ $\left.\mathrm{Hz}, \mathrm{OCH}_{2} \mathrm{Ph}\right), 4.85\left(\mathrm{~d}, 1 \mathrm{H}, J=12.6 \mathrm{~Hz}, \mathrm{OCH}_{2} \mathrm{Ph}\right), 4.81(\mathrm{~d}, 1 \mathrm{H}, J=$ $12.7 \mathrm{~Hz}, \mathrm{OCH}_{2} \mathrm{Ph}$ ), $4.72\left(\mathrm{~d}, 1 \mathrm{H}, J=12.6 \mathrm{~Hz}, \mathrm{OCH}_{2} \mathrm{Ph}\right), 4.34-4.28$ (m, $2 \mathrm{H}, \mathrm{H}-4, \mathrm{H}-5), 4.22$ (dd, $1 \mathrm{H}, J=5.4,7.7 \mathrm{~Hz}, \mathrm{H}-3), 4.15$ (dd, $1 \mathrm{H}$, $\left.J=7.9,12.8 \mathrm{~Hz}, \mathrm{H}-5^{\prime}\right), 3.92(\mathrm{dd}, 1 \mathrm{H}, J=7.5,5.4 \mathrm{~Hz}, \mathrm{H}-2), 2.31(\mathrm{~s}$, $\left.3 \mathrm{H}, \mathrm{ArCH}_{3}\right), 2.02\left(\mathrm{~s}, 3 \mathrm{H}, \mathrm{C}(\mathrm{O}) \mathrm{CH}_{3}\right) ;{ }^{13} \mathrm{C} \mathrm{NMR}\left(125 \mathrm{MHz}, \mathrm{CDCl}_{3}\right)$ $\delta_{\mathrm{C}} 170.9(\mathrm{C}=\mathrm{O}), 137.9(\mathrm{Ar}), 136.2(\mathrm{Ar}), 135.6(\mathrm{Ar}), 132.9(\mathrm{Ar})$,
131.7 (Ar), 131.4 (Ar), 129.8 (Ar), 129.7 (Ar), 129.7 (Ar), 129.6 (Ar), 129.5 (Ar), 88.9 (C-1), 84.9 (C-2), 80.7 (C-3), 78.5 (C-4), 69.9 $\left(\mathrm{OCH}_{2} \mathrm{Ph}\right), 68.8\left(\mathrm{OCH}_{2} \mathrm{Ph}\right), 63.6(\mathrm{C}-5), 21.2\left(\mathrm{ArCH}_{3}\right), 21.1(\mathrm{C}(\mathrm{O})$ $\left.\mathrm{CH}_{3}\right)$; HRMS-ESI $m / z[\mathrm{M}+\mathrm{Na}]^{+}$calcd for $\mathrm{C}_{22} \mathrm{H}_{24} \mathrm{NaO}_{5} \mathrm{~S}: 423.1242$. Found: 423.1248.

p-Tolyl 1-Thio-2,3-O-xylylene- $\beta$-D-xylofuranoside (12). To a solution of $11^{18}(2.26 \mathrm{~g}, 4.56 \mathrm{mmol})$ in DMF $(40 \mathrm{~mL})$ were added $\mathrm{NaH}(400 \mathrm{mg}, 10.04 \mathrm{mmol})$ and $\alpha, \alpha^{\prime}$-dibromo-o-xylene (1.32 g, 5.02 $\mathrm{mmol}$ ) slowly at $0{ }^{\circ} \mathrm{C}$. After stirring for at $2 \mathrm{~h}$, the reaction was quenched by the addition of satd aq. $\mathrm{NH}_{4} \mathrm{Cl}$. Dilution of the mixture with $\mathrm{CH}_{2} \mathrm{Cl}_{2}$ provided a solution that was then washed with brine. The organic layer was dried with anhydrous $\mathrm{Na}_{2} \mathrm{SO}_{4}$, filtered, and concentrated. The crude product was purified by flash column chromatography (20:1 hexane-EtOAc) to give the crude product. The obtained crude product was dissolved in $\mathrm{CH}_{2} \mathrm{Cl}_{2}-$ methanol (7:3, $20 \mathrm{~mL}$ ), and then $p$-TsOH $(80 \mathrm{mg})$ was added. The mixture was stirred for $14 \mathrm{~h}$ and then neutralized by the addition of $\mathrm{Et}_{3} \mathrm{~N}$. Then the mixture was concentrated to syrup, which was purified by column chromatography (6:1 hexane-EtOAc) to give $\mathbf{1 2}$ (990 mg, 41\% over two steps) as a colorless oil. $R_{\mathrm{f}} 0.3\left(3: 1\right.$, hexanes-EtOAc); $[\alpha]_{\mathrm{D}}=$ $-78.3\left(c=0.9, \mathrm{CHCl}_{3}\right) ;{ }^{1} \mathrm{H} \mathrm{NMR}\left(500 \mathrm{MHz}, \mathrm{CDCl}_{3}\right) \delta_{\mathrm{H}} 7.43-7.32$ (m, 6H, ArH), 7.10-7.06 (m, 2H, ArH), $5.07(\mathrm{~d}, 1 \mathrm{H}, J=7.3 \mathrm{~Hz}, \mathrm{H}-$ 1), $4.93\left(\mathrm{~d}, 1 \mathrm{H}, J=12.7 \mathrm{~Hz}, \mathrm{OCH}_{2} \mathrm{Ph}\right), 4.92(\mathrm{~d}, 1 \mathrm{H}, J=12.5 \mathrm{~Hz}$, $\left.\mathrm{OCH}_{2} \mathrm{Ph}\right), 4.82\left(\mathrm{~d}, 1 \mathrm{H}, J=12.7 \mathrm{~Hz}, \mathrm{OCH}_{2} \mathrm{Ph}\right), 4.75(\mathrm{~d}, 1 \mathrm{H}, J=12.6$ $\mathrm{Hz}, \mathrm{OCH}_{2} \mathrm{Ph}$ ), 4.26 (dd, $1 \mathrm{H}, J=5.4,7.9 \mathrm{~Hz}, \mathrm{H}-3$ ), 4.16 (ddd, $1 \mathrm{H}, J=$ 7.9, 5.1. 5.1 Hz, H-4), 4.04 (dd, $1 \mathrm{H}, J=7.3,5.4 \mathrm{~Hz}, \mathrm{H}-2), 3.76-3.73$ (m, 2H, H-5), 2.34 (app t, 1H, J = 7.1 Hz, OH), $2.31\left(\mathrm{~s}, 3 \mathrm{H} \mathrm{ArCH}_{3}\right)$; ${ }^{13} \mathrm{C}$ NMR $\left(125 \mathrm{MHz}, \mathrm{CDCl}_{3}\right) \delta_{\mathrm{C}} 137.8(\mathrm{Ar}), 136.3(\mathrm{Ar}), 135.2(\mathrm{Ar})$, 132.4 (2C, Ar), 131.7 (Ar), 131.4 (Ar), 129.9 (Ar), 129.7 (Ar), 129.7 (2C, Ar), 129.6 (Ar), 89.0 (C-1), 85.3 (C-2), 81.4 (C-3), 80.6 (C-4), $69.8\left(\mathrm{OCH}_{2} \mathrm{Ph}\right), 68.90\left(\mathrm{OCH}_{2} \mathrm{Ph}\right), 61.8(\mathrm{C}-5), 21.1\left(\mathrm{ArCH}_{3}\right)$; HRMS-ESI $\mathrm{m} / z[\mathrm{M}+\mathrm{Na}]^{+}$calcd for $\mathrm{C}_{20} \mathrm{H}_{22} \mathrm{NaO}_{4} \mathrm{~S}: 381.1131$. Found: 381.1128 .

5-O-p-Methoxybenzyl-2,3-O-xylylene- $\alpha$-D-xylofuranosyl- $(1 \rightarrow 6)$ 1,2:3,4-di-O-isopropylidene- $\alpha$-D-galactopyranose (14). $R_{\mathrm{f}} 0.40$ (2:1, hexanes-EtOAc); $[\alpha]_{\mathrm{D}}=+48.9\left(c=0.9, \mathrm{CHCl}_{3}\right) ;{ }^{1} \mathrm{H}$ NMR (500 $\left.\mathrm{MHz}^{\mathrm{CDCl}}\right)_{3} \delta_{\mathrm{H}} 7.41-7.37(\mathrm{~m}, 2 \mathrm{H}, \mathrm{ArH}), 7.36-7.31(\mathrm{~m}, 2 \mathrm{H}, \mathrm{ArH})$, 7.28-7.23 (m, 2H, ArH), 6.88-6.83 (m, 2H, ArH), $5.52(\mathrm{~d}, 1 \mathrm{H}, J=$ $\left.5.0 \mathrm{~Hz}, \mathrm{H}-1^{\prime}\right), 4.94(\mathrm{~d}, 1 \mathrm{H}, J=2.5 \mathrm{~Hz}, \mathrm{H}-1), 4.88(\mathrm{~d}, 1 \mathrm{H}, J=12.5 \mathrm{~Hz}$, $\mathrm{OCH}_{2} \mathrm{Ph}$ ), $4.85\left(\mathrm{~d}, 1 \mathrm{H}, J=12.7 \mathrm{~Hz}, \mathrm{OCH}_{2} \mathrm{Ph}\right), 4.74(\mathrm{~d}, 1 \mathrm{H}, J=12.7$ $\left.\mathrm{Hz}, \mathrm{OCH}_{2} \mathrm{Ph}\right), 4.71\left(\mathrm{~d}, 1 \mathrm{H}, J=12.5 \mathrm{~Hz}, \mathrm{OCH}_{2} \mathrm{Ph}\right), 4.53(\mathrm{dd}, 1 \mathrm{H}, J=$ 2.4, 7.9 Hz, H-3'), $4.51\left(\mathrm{~d}, 1 \mathrm{H}, J=11.6 \mathrm{~Hz}, \mathrm{OCH}_{2} \mathrm{Ph}\right), 4.43(\mathrm{~d}, 1 \mathrm{H}, J$ $=11.6 \mathrm{~Hz}, \mathrm{OCH}_{2} \mathrm{Ph}$ ), 4.37 (ddd, $1 \mathrm{H}, J=5.0,7.2,7.2 \mathrm{~Hz}, \mathrm{H}-5$ ), 4.28 $\left(\mathrm{dd}, 1 \mathrm{H}, J=5.0,2.8 \mathrm{~Hz}, \mathrm{H}-2^{\prime}\right), 4.18-4.12(\mathrm{~m}, 2 \mathrm{H}, \mathrm{H}-2, \mathrm{H}-4), 4.09$ (ddd, $J=1.6,7.2 \mathrm{~Hz}, \mathrm{H}-4^{\prime}$ ), 3.95-3.88 (m, 2H, H-3, H-5'), 3.79 (s, $\left.3 \mathrm{H}, \mathrm{OCH}_{3}\right), 3.69-3.64\left(\mathrm{~m}, 2 \mathrm{H}, \mathrm{H}-6^{\prime}, \mathrm{H}-6\right), 3.61(\mathrm{dd}, 1 \mathrm{H}, J=4.6,9.3$ $\left.\mathrm{Hz}, \mathrm{H}-5^{\prime}\right), 1.54$ (s, 3H, $\left.\mathrm{CH}_{3}\right), 1.39$ (s, $\left.3 \mathrm{H}, \mathrm{CH}_{3}\right), 1.33$ (s, 3H, $\left.\mathrm{CH}_{3}\right)$, $1.28\left(\mathrm{~s}, 3 \mathrm{H}, \mathrm{CH}_{3}\right) ;{ }^{13} \mathrm{C} \mathrm{NMR}\left(125 \mathrm{MHz}, \mathrm{CDCl}_{3}\right) \delta_{\mathrm{C}} 159.1(\mathrm{Ar})$, 136.5 (Ar), $135.6(\mathrm{Ar}), 131.6(\mathrm{Ar}), 131.5(\mathrm{Ar}), 130.8(\mathrm{Ar}), 129.6$ (Ar), $129.6(\mathrm{Ar}), 129.2(\mathrm{Ar}), 113.7(\mathrm{Ar}), 109.2\left(\left(\mathrm{CH}_{3}\right)_{2} \mathrm{CO}_{2}\right), 108.5$ $\left(\left(\mathrm{CH}_{3}\right)_{2} \mathrm{CO}_{2}\right), 108.2(\mathrm{C}-1), 96.3\left(\mathrm{C}-1^{\prime}\right), 87.2(\mathrm{C}-2), 81.6(\mathrm{C}-4), 80.1$ (C-5), $72.8\left(\mathrm{OCH}_{2} \mathrm{Ph}\right), 71.1\left(\mathrm{C}-4^{\prime}\right), 70.6\left(\mathrm{C}-2^{\prime}\right), 70.6\left(\mathrm{C}-3^{\prime}\right), 70.1$ (C-6), $69.3\left(\mathrm{OCH}_{2} \mathrm{Ph}\right), 69.1\left(\mathrm{OCH}_{2} \mathrm{Ph}\right), 67.0(\mathrm{C}-3), 66.9\left(\mathrm{C}-5^{\prime}\right)$, $55.2\left(\mathrm{OCH}_{3}\right), 26.1\left(\mathrm{CH}_{3}\right), 26.0\left(\mathrm{CH}_{3}\right), 25.0\left(\mathrm{CH}_{3}\right), 24.4\left(\mathrm{CH}_{3}\right)$; HRMS-ESI $\mathrm{m} / z[\mathrm{M}+\mathrm{Na}]^{+}$calcd for $\mathrm{C}_{33} \mathrm{H}_{42} \mathrm{NaO}_{11}: 637.2625$. Found: 637.2633 .

5-O-Acetyl-2,3-O-xylylene- $\alpha$-D-xylofuranosyl- $(1 \rightarrow 6)-1,2: 3,4-d i-O$ isopropylidene- $\alpha$-D-galactopyranose (15). $R_{\mathrm{f}} 0.40(1: 1$, hexanesEtOAc); $[\alpha]_{\mathrm{D}}=+23.5\left(c=0.3, \mathrm{CHCl}_{3}\right) ; \mathrm{H} \mathrm{NMR}\left(500 \mathrm{MHz}, \mathrm{CDCl}_{3}\right)$ $\delta_{\mathrm{H}} 7.42-7.31(\mathrm{~m}, 4 \mathrm{H}, \mathrm{ArH}), 5.51\left(\mathrm{~d}, 1 \mathrm{H}, J=5.0 \mathrm{~Hz}, \mathrm{H}-1^{\prime}\right), 5.06-$ $5.00\left(\mathrm{~m}, 2 \mathrm{H}, \mathrm{H}-1, \mathrm{OCH}_{2} \mathrm{Ph}\right), 4.83\left(\mathrm{~d}, 1 \mathrm{H}, J=10.1 \mathrm{~Hz}, \mathrm{OCH}_{2} \mathrm{Ph}\right)$, $4.81\left(\mathrm{~d}, 1 \mathrm{H}, J=12.6 \mathrm{~Hz}, \mathrm{OCH}_{2} \mathrm{Ph}\right), 4.71(\mathrm{~d}, 1 \mathrm{H}, J=12.6 \mathrm{~Hz}$, $\mathrm{OCH}_{2} \mathrm{Ph}$ ), $4.58\left(\mathrm{dd}, 1 \mathrm{H}, J=2.4,7.9 \mathrm{~Hz}, \mathrm{H}-3^{\prime}\right), 4.50(\mathrm{dd}, J=2.8,7.9$ $\mathrm{Hz}, \mathrm{H}-4^{\prime}$ ), 4.38 (dd, $\left.1 \mathrm{H}, J=2.8,12.3 \mathrm{~Hz}, \mathrm{H}-6^{\prime}\right), 4.34$ (dd, $1 \mathrm{H}, J=5.0$, $2.4 \mathrm{~Hz}, \mathrm{H}-4), 4.29$ (dd, $\left.1 \mathrm{H}, J=5.0,2.4 \mathrm{~Hz}, \mathrm{H}-2^{\prime}\right), 4.24(\mathrm{dd}, 1 \mathrm{H}, J=$ 2.0, $7.9 \mathrm{~Hz}, \mathrm{H}-3), 4.12-4.10$ (m, $1 \mathrm{H}, \mathrm{H}-6), 4.06-3.99$ (m, $2 \mathrm{H}, \mathrm{H}-2$, $\mathrm{H}-5), 3.85-3.76\left(\mathrm{~m}, 2 \mathrm{H}, \mathrm{H}-5^{\prime}, \mathrm{H}-5^{\prime}\right), 2.02\left(\mathrm{~s}, 3 \mathrm{H}, \mathrm{C}(\mathrm{O}) \mathrm{CH}_{3}\right), 1.52$ $\left(\mathrm{s}, 3 \mathrm{H}, \mathrm{CH}_{3}\right), 1.30\left(\mathrm{~s}, 3 \mathrm{H}, \mathrm{CH}_{3}\right), 1.29\left(\mathrm{~s}, 3 \mathrm{H}, \mathrm{CH}_{3}\right), 1.19(\mathrm{~s}, 3 \mathrm{H}$, $\left.\mathrm{CH}_{3}\right) ;{ }^{13} \mathrm{C}$ NMR $\left(125 \mathrm{MHz}, \mathrm{CDCl}_{3}\right) \delta_{\mathrm{C}} 170.9(\mathrm{C}=\mathrm{O}), 136.6(\mathrm{Ar})$, 135.5 (Ar), 131.6 (Ar), 131.5 (Ar), 129.6 (Ar), 129.5 (Ar), 109.2 (C- 
1), 96.3 (C-1'), 83.1 (C-2), 80.0 (C-2'), 76.1 (C-4), 71.4 (C-3), 70.7 $\left(\mathrm{C}-3^{\prime}\right), 70.6(\mathrm{C}-5), 69.7\left(\mathrm{OCH}_{2} \mathrm{Ph}\right), 69.1\left(\mathrm{OCH}_{2} \mathrm{Ph}\right), 67.0\left(\mathrm{C}-5^{\prime}\right)$, 66.8 (C-4'), 63.6 (C-6), $26.07\left(\mathrm{CH}_{3}\right), 25.82\left(\mathrm{CH}_{3}\right), 24.96\left(\mathrm{CH}_{3}\right)$, $24.21\left(\mathrm{CH}_{3}\right) 21.0\left(\mathrm{C}(\mathrm{O}) \mathrm{CH}_{3}\right)$; HRMS-ESI $m / z[\mathrm{M}+\mathrm{Na}]^{+}$calcd for $\mathrm{C}_{27} \mathrm{H}_{36} \mathrm{NaO}_{11}$ : 559.2155. Found: 559.2164.

Benzyl 5-O-Acetyl-2,3-O-xylylene- $\alpha$-D-xylofuranosyl- $(1 \rightarrow 5)-2,3-$ O-isopropylidene- $\beta$-D-ribofuranoside (25). $R_{\mathrm{f}} 0.3$ (2:1, hexaneEtOAc); $[\alpha]_{\mathrm{D}}=-24.5\left(c=0.5, \mathrm{CH}_{2} \mathrm{Cl}_{2}\right) ;{ }^{1} \mathrm{H}$ NMR $(500 \mathrm{MHz}$, $\left.\mathrm{CDCl}_{3}\right) \delta_{\mathrm{H}} 7.41-7.27(\mathrm{~m}, 9 \mathrm{H}, \mathrm{ArH}), 5.13(\mathrm{~s}, 1 \mathrm{H}, \mathrm{H}-1), 5.03-5.00$ $\left(\mathrm{m}, 2 \mathrm{H}, \mathrm{H}-1^{\prime}, \mathrm{OCH}_{2} \mathrm{Ph}\right), 4.83\left(\mathrm{~d}, 1 \mathrm{H}, J=11.8 \mathrm{~Hz}, \mathrm{OCH}_{2} \mathrm{Ph}\right), 4.83$ (d, $1 \mathrm{H}, J=12.7 \mathrm{~Hz}, \mathrm{OCH}_{2} \mathrm{Ph}$ ), 4.79 (dd, $1 \mathrm{H}, J=6.0,1.0 \mathrm{~Hz}, \mathrm{H}-3$ ), $4.71\left(\mathrm{~d}, 1 \mathrm{H}, J=12.7 \mathrm{~Hz}, \mathrm{OCH}_{2} \mathrm{Ph}\right), 4.67(\mathrm{~d}, 1 \mathrm{H}, J=11.8 \mathrm{~Hz}$, $\mathrm{OCH}_{2} \mathrm{Ph}$ ), 4.65 (d, $\left.1 \mathrm{H}, J=6.0 \mathrm{~Hz}, \mathrm{H}-2\right)$, 4.50-4.43 (m, 2H, H-4', H4), $4.42\left(\mathrm{~d}, 1 \mathrm{H}, J=11.8 \mathrm{~Hz}, \mathrm{OCH}_{2} \mathrm{Ph}\right), 4.39-4.36\left(\mathrm{~m}, 2 \mathrm{H}, \mathrm{H}-3^{\prime}, \mathrm{H}-\right.$ $\left.5^{\prime}\right), 4.12\left(\mathrm{dd}, 1 \mathrm{H}, J=12.3,8.2 \mathrm{~Hz}, \mathrm{H}-5^{\prime}\right), 4.01(\operatorname{app~t}, 1 \mathrm{H}, J=4.9 \mathrm{~Hz}$, $\left.\mathrm{H}-2^{\prime}\right), 3.75-3.70\left(\mathrm{~m}, 2 \mathrm{H}, \mathrm{H}-5^{\prime}, \mathrm{H}-5 \mathrm{~B}\right), 1.97$ (s, 3H, C(O) $\left.\mathrm{CH}_{3}\right), 1.46$ $\left(\mathrm{s}, 3 \mathrm{H}, \mathrm{CH}_{3}\right), 1.31\left(\mathrm{~s}, 3 \mathrm{H}, \mathrm{CH}_{3}\right) ;{ }^{13} \mathrm{C}$ NMR $\left(125 \mathrm{MHz}, \mathrm{CDCl}_{3}\right) \delta_{\mathrm{C}}$ $170.8(\mathrm{C}=\mathrm{O}), 137.1$ (Ar), 136.5 (Ar), $135.4(\mathrm{Ar}), 131.6(\mathrm{Ar}), 131.5$ (Ar), $129.6(\mathrm{Ar}), 129.5(\mathrm{Ar}), 128.4(\mathrm{Ar}), 127.9$ (2C, Ar), 127.8 (2C, $\mathrm{Ar}), 107.2$ (C-1), $101.9\left(\mathrm{C}-1^{\prime}\right), 85.4$ (C-4' or C-4), 85.3 (C-2), 83.0 $\left(\mathrm{C}-2^{\prime}\right), 82.1(\mathrm{C}-3), 79.7\left(\mathrm{C}-3^{\prime}\right), 76.3\left(\mathrm{C}-4^{\prime}\right.$ or $\left.\mathrm{C}-4\right), 69.7\left(\mathrm{OCH}_{2} \mathrm{Ph}\right)$, $69.6(\mathrm{C}-5), 69.04\left(\mathrm{OCH}_{2} \mathrm{Ph}\right), 69.03\left(\mathrm{OCH}_{2} \mathrm{Ph}\right), 63.5\left(\mathrm{C}-5^{\prime}\right), 26.5$ $\left(\mathrm{CH}_{3}\right), 25.1\left(\mathrm{CH}_{3}\right), 20.9\left(\mathrm{C}(\mathrm{O}) \mathrm{CH}_{3}\right)$; HRMS-ESI $m / z[\mathrm{M}+\mathrm{Na}]^{+}$ calcd for $\mathrm{C}_{30} \mathrm{H}_{36} \mathrm{NaO}_{10}$ : 579.2206. Found: 579.2210.

p-Methoxyphenyl 5-O-acetyl-2,3-O-xylylene- $\alpha$-D-xylofuranosyl$(1 \rightarrow 4)-6-0$ - $t$-butyl-diphenylsilyl-2,3-di-O-benzoyl- $\beta$-D-galactopyranoside (26). $R_{\mathrm{f}} 0.3\left(4: 1\right.$, hexane-EtOAc); $[\alpha]_{\mathrm{D}}=+45.7(c=0.9$, $\left.\mathrm{CHCl}_{3}\right) ;{ }^{1} \mathrm{H}$ NMR $\left(500 \mathrm{MHz}, \mathrm{CDCl}_{3}\right) \delta_{\mathrm{H}} 8.04-7.96(\mathrm{~m}, 4 \mathrm{H}, \mathrm{ArH})$, 7.80-7.73 (m, 4H, ArH), 7.54-7.28 (m, 15H, ArH), 7.19-7.12 (m, $1 \mathrm{H}, \mathrm{ArH}), 7.01-6.93(\mathrm{~m}, 2 \mathrm{H}, \mathrm{ArH}), 6.76-6.69(\mathrm{~m}, 2 \mathrm{H}, \mathrm{ArH}), 5.96$ (dd, $1 \mathrm{H}, J=8.0,10.6 \mathrm{~Hz}, \mathrm{H}-2), 5.24$ (dd, $1 \mathrm{H}, J=10.6,3.3 \mathrm{~Hz}, \mathrm{H}-3$ ), $5.12(\mathrm{~d}, 1 \mathrm{H}, J=8.0 \mathrm{~Hz}, \mathrm{H}-1), 5.12\left(\mathrm{~d}, 1 \mathrm{H}, J=4.6 \mathrm{~Hz}, \mathrm{H}-1^{\prime}\right), 4.85(\mathrm{~d}$, $\left.1 \mathrm{H}, J=12.8 \mathrm{~Hz}, \mathrm{OCH}_{2} \mathrm{Ph}\right), 4.76-4.66\left(\mathrm{~m}, 2 \mathrm{H}, \mathrm{OCH}_{2} \mathrm{Ph}\right), 4.50-4.42$ (m, $4 \mathrm{H}, \mathrm{H}-3^{\prime}, \mathrm{H}-4^{\prime}, \mathrm{H}-4, \mathrm{OCH}_{2} \mathrm{Ph}$ ), 4.15 (dd, $1 \mathrm{H}, J=10.6,6.5 \mathrm{~Hz}$, H-6'), 4.09 (dd, 1H, $J=10.6,6.4 \mathrm{~Hz}, \mathrm{H}-6$ ), 3.92 (app t, 1H, $J=6.4$ $\mathrm{Hz}, \mathrm{H}-5$ ), 3.81 (app t, $1 \mathrm{H}, J=4.8 \mathrm{~Hz}, \mathrm{H}-2^{\prime}$ ), 3.73 (s, $3 \mathrm{H}, \mathrm{OCH}_{3}$ ), $3.67\left(\mathrm{dd}, 1 \mathrm{H}, J=11.8,3.0 \mathrm{~Hz}, \mathrm{H}-5^{\prime}\right), 3.55(\mathrm{dd}, 1 \mathrm{H}, J=11.8,3.8 \mathrm{~Hz}$, $\left.\mathrm{H}^{-5^{\prime}}\right), 1.71\left(\mathrm{~s}, 3 \mathrm{H}, \mathrm{C}(\mathrm{O}) \mathrm{CH}_{3}\right), 1.12\left(\mathrm{~s}, 9 \mathrm{H}, 3 \times \mathrm{CH}_{3}\right) ;{ }^{13} \mathrm{C} \mathrm{NMR}$ $\left(125 \mathrm{MHz}, \mathrm{CDCl}_{3}\right) \delta_{\mathrm{C}} 170.4(\mathrm{C}=\mathrm{O}), 166.0(\mathrm{C}=\mathrm{O}), 165.4(\mathrm{C}=$ O), 155.3 (Ar), 151.4 (Ar), 137.1 (Ar), 135.7 (Ar), 135.65 (2C, Ar), 135.62 (2C, Ar), 133.4 (Ar), 133.3 (Ar), 133.2 (Ar), 133.1 (Ar), 131.4 (Ar), 131.1 (Ar), 129.9 (Ar), 129.80 (Ar), $129.79(\mathrm{Ar}), 129.6$ (2C, Ar), 129.5 (Ar), 129.2 (Ar), 129.1 (Ar), 128.38 (2C, Ar), 128.32 (2C, Ar), 127.83 (2C, Ar), 127.81 (2C, Ar), 118.6 (2C, Ar), 114.4 (2C, Ar), 103.3 (C-1'), 101.0 (C-1), 82.1 (C-2'), 79.4, 76.0, 75.7, 74.7 (C-3', C-4', C-4, C-5), 73.8 (C-3), $69.6(\mathrm{C}-2), 69.3\left(\mathrm{OCH}_{2} \mathrm{Ph}\right)$, $69.0\left(\mathrm{OCH}_{2} \mathrm{Ph}\right), 62.2(\mathrm{C}-6), 62.0\left(\mathrm{C}-5^{\prime}\right), 55.5\left(\mathrm{OCH}_{3}\right), 26.9$ $\left(\mathrm{C}\left(\mathrm{CH}_{3}\right)_{3}\right), 20.7\left(\mathrm{C}(\mathrm{O}) \mathrm{CH}_{3}\right), 19.2\left(3 \times \mathrm{CH}_{3}\right) ;$ HRMS-ESI $\mathrm{m} / z[\mathrm{M}+$ $\mathrm{Na}]^{+}$calcd for $\mathrm{C}_{58} \mathrm{H}_{60} \mathrm{NaO}_{14} \mathrm{Si}$ : 1031.3650 . Found: 1031.3661 .

Methyl 5-O-Acetyl-2,3-O-xylylene- $\alpha$-D-xylofuranosyl- $(1 \rightarrow 6)$ 2,3,4-tri-O-benzoyl- $\alpha$-D-glucopyranoside (27). $R_{\mathrm{f}} 0.4$ (3:2 hexaneEtOAc); $[\alpha]_{\mathrm{D}}=+45.3\left(c=0.7, \mathrm{CHCl}_{3}\right) ;{ }^{1} \mathrm{H}$ NMR $(500 \mathrm{MHz}$, $\left.\mathrm{CDCl}_{3}\right) \delta_{\mathrm{H}} 8.01-7.96(\mathrm{~m}, 2 \mathrm{H}, \mathrm{ArH}), 7.96-7.92(\mathrm{~m}, 2 \mathrm{H}, \mathrm{ArH})$, 7.89-7.83 (m, 2H, ArH), 7.53-7.46 (m, 2H, ArH), 7.43-7.27 (m, $11 \mathrm{H}, \mathrm{ArH}), 6.13($ app t, $1 \mathrm{H}, J=9.8 \mathrm{~Hz}, \mathrm{H}-3), 5.58$ (app t, $1 \mathrm{H}, J=9.8$ $\mathrm{Hz}, \mathrm{H}-4), 5.29$ (dd, $1 \mathrm{H}, J=3.6,9.8 \mathrm{~Hz}, \mathrm{H}-2), 5.22(\mathrm{~d}, 1 \mathrm{H}, J=3.6 \mathrm{~Hz}$, $\mathrm{H}-1), 4.98-4.91\left(\mathrm{~m}, 2 \mathrm{H}, \mathrm{H}-1^{\prime}, \mathrm{OCH}_{2} \mathrm{Ph}\right), 4.79(\mathrm{~d}, 1 \mathrm{H}, J=12.7 \mathrm{~Hz}$, $\mathrm{OCH}_{2} \mathrm{Ph}$ ), 4.76-4.68 (m, $\left.2 \mathrm{H}, \mathrm{OCH}_{2} \mathrm{Ph}\right), 4.47$ (ddd, $1 \mathrm{H}, J=2.7,8.0$ $\left.\mathrm{Hz}, \mathrm{H}-4^{\prime}\right), 4.36-4.30\left(\mathrm{~m}, 2 \mathrm{H}, \mathrm{H}-3^{\prime}, \mathrm{H}-5^{\prime}\right), 4.27$ (ddd, $1 \mathrm{H}, J=9.8$, 5.6, $2.8 \mathrm{~Hz}, \mathrm{H}-5$ ), 4.07 (dd, $1 \mathrm{H}, J=12.3,8.0 \mathrm{~Hz}, \mathrm{H}-5^{\prime}$ ), 3.97 (app t, $\left.1 \mathrm{H}, J=5.0 \mathrm{~Hz}, \mathrm{H}-2^{\prime}\right), 3.92\left(\mathrm{dd}, 1 \mathrm{H}, J=11.1,5.6 \mathrm{~Hz}, \mathrm{H}-6^{\prime}\right.$ ), 3.70 (dd, $1 \mathrm{H}, J=11.1,2.8 \mathrm{~Hz}, \mathrm{H}-6), 3.44\left(\mathrm{~s}, 3 \mathrm{H}, \mathrm{OCH}_{3}\right), 1.90(\mathrm{~s}, 3 \mathrm{H}$, $\left.\mathrm{C}(\mathrm{O}) \mathrm{CH}_{3}\right) ;{ }^{13} \mathrm{C}$ NMR $\left(125 \mathrm{MHz}, \mathrm{CDCl}_{3}\right) \delta_{\mathrm{C}} 170.9(\mathrm{C}=\mathrm{O}), 165.8$ $(\mathrm{C}=\mathrm{O}), 165.8(\mathrm{C}=\mathrm{O}), 165.3(\mathrm{C}=\mathrm{O}), 136.8(\mathrm{Ar}), 135.5(\mathrm{Ar})$, 133.3 (Ar), 133.3 (Ar), $133.0(\mathrm{Ar}), 131.6$ (Ar), $131.4(\mathrm{Ar}), 129.9$ (2C, Ar), 129.8 (2C, Ar), 129.6 (2C, Ar), 129.5 (Ar), 129.4 (Ar), 129.3 (Ar), 129.1 (Ar), 129.1 (Ar), 128.4 (3C, Ar), 128.2 (2C, Ar), 100.8 (C-1'), 96.8 (C-1), $82.7\left(\mathrm{C}-2^{\prime}\right), 79.9\left(\mathrm{C}-3^{\prime}\right), 76.2\left(\mathrm{C}-4^{\prime}\right), 72.1$ (C-2), $70.7(\mathrm{C}-3), 69.7(\mathrm{C}-4), 69.3\left(\mathrm{OCH}_{2} \mathrm{Ph}\right), 69.3\left(\mathrm{OCH}_{2} \mathrm{Ph}\right), 68.3$ (C-6), 66.2 (C-5), $63.6\left(\mathrm{C}-5^{\prime}\right), 55.5\left(\mathrm{OCH}_{3}\right), 20.8\left(\mathrm{C}(\mathrm{O}) \mathrm{CH}_{3}\right)$;
HRMS-ESI $m / z[\mathrm{M}+\mathrm{Na}]^{+}$calcd for $\mathrm{C}_{43} \mathrm{H}_{42} \mathrm{NaO}_{14}$ : 805.2472, Found: 805.2479.

p-Methoxyphenyl 5-O-Acetyl-2,3-O-xylylene- $\alpha$-D-xylofuranosyl$(1 \rightarrow 3)$-4-O-acetyl-2,6-di-O-benzoyl- $\beta$-D-galactopyranosyl- $(1 \rightarrow 4)$ 2,3,6-tri-O-benzoyl- $\beta$-D-glucopyranoside (28). $R_{\mathrm{f}} 0.4$ (3:2, hexaneEtOAc); $[\alpha]_{\mathrm{D}}=+27.3\left(c=1.8, \mathrm{CHCl}_{3}\right) ;{ }^{1} \mathrm{H} \mathrm{NMR}(500 \mathrm{MHz}$, $\left.\mathrm{CDCl}_{3}\right) \delta_{\mathrm{H}} 8.07-7.99(\mathrm{~m}, 6 \mathrm{H}, \mathrm{ArH}), 7.99-7.88(\mathrm{~m}, 4 \mathrm{H}, \mathrm{ArH})$, 7.61-7.48 (m, 6H, ArH), 7.42-7.30 (m, 11H, ArH), 7.25-7.18 (m, $2 \mathrm{H}, \mathrm{ArH}), 6.89-6.81(\mathrm{~m}, 2 \mathrm{H}, \mathrm{ArH}), 6.66-6.60(\mathrm{~m}, 2 \mathrm{H}, \mathrm{ArH}), 5.79$ (app t, $1 \mathrm{H}, J=9.3 \mathrm{~Hz}, \mathrm{H}-3), 5.64(\mathrm{dd}, 1 \mathrm{H}, J=9.3,7.8 \mathrm{~Hz}, \mathrm{H}-2)$, 5.37-5.29 (m, 2H, H-2', H-4'), $5.09(\mathrm{~d}, 1 \mathrm{H}, J=7.8 \mathrm{~Hz}, \mathrm{H}-1), 4.97$ $\left(\mathrm{d}, 1 \mathrm{H}, J=4.6 \mathrm{~Hz}, \mathrm{H}-1^{\prime \prime}\right), 4.84\left(\mathrm{~d}, 1 \mathrm{H}, J=13.1 \mathrm{~Hz}, \mathrm{OCH}_{2} \mathrm{Ph}\right), 4.68-$ $4.52\left(\mathrm{~m}, 5 \mathrm{H}, 3 \times \mathrm{OCH}_{2} \mathrm{Ph}, \mathrm{H}-1^{\prime}, \mathrm{H}-5 \mathrm{~b}^{\prime \prime}\right), 4.41(\mathrm{dd}, 1 \mathrm{H}, J=11.9,5.6$ $\mathrm{Hz}, \mathrm{H}-5 \mathrm{a}^{\prime \prime}$ ), 4.20 (app t, 1H, $\left.J=9.5 \mathrm{~Hz}, \mathrm{H}-4\right), 3.98-3.96(\mathrm{~m}, 1 \mathrm{H}, \mathrm{H}-$ $\left.3^{\prime}\right)$, 3.95-3.94 (m, 1H, H-3"), 3.93-3.81 (m, 5H, H-2", H-4", H-5, H-6a, H-6b), 3.74-3.60 (m, 6H, H-5', H-6a', H-6b', OCH ${ }_{3}$ ), 2.09 (s, $\left.3 \mathrm{H}, \mathrm{C}(\mathrm{O}) \mathrm{CH}_{3}\right), 1.87\left(\mathrm{~s}, 3 \mathrm{H}, \mathrm{C}(\mathrm{O}) \mathrm{CH}_{3}\right) ;{ }^{13} \mathrm{C}$ NMR $(125 \mathrm{MHz}$, $\left.\mathrm{CDCl}_{3}\right) \delta_{\mathrm{C}} 165.7(\mathrm{C}=\mathrm{O}), 165.6(\mathrm{C}=\mathrm{O}), 165.2(\mathrm{C}=\mathrm{O}), 164.6$ $(\mathrm{C}=\mathrm{O}), 155.6(\mathrm{C}=\mathrm{O}), 136.2(\mathrm{Ar}), 135.9(\mathrm{Ar}), 133.4(2 \mathrm{C}, \mathrm{Ar})$, 133.3 (2C, Ar), 133.2 (2C, Ar), 133.1 (2C, Ar), 131.6 (2C, Ar), 131.2 (2C, Ar), 129.85 (2C, Ar), 129.81 (2C, Ar), 129.7 (2C, Ar), 129.66 (2C, Ar), 129.64 (2C, Ar), 129.5 (2C, Ar), 129.3 (2C, Ar), 129.2 (Ar), 128.6 (2C, Ar), 128.5 (2C, Ar), 128.47 (2C, Ar), 128.43 (2C, Ar), 128.2 (2C, Ar), 118.9 (2C, Ar), 114.4 (2C, Ar), 101.1 (C-1'), 100.6 (C-1C), $97.5\left(\mathrm{C}-1^{\prime \prime}\right), 81.8$ (C-2"), $79.1\left(\mathrm{C}-3^{\prime \prime}\right), 76.1$ (C-4), 75.8 (C-5), 73.1 (C-3'), 72.9 (C-3), 72.8 (C-4"), 71.6 (C-2), 71.1 $\left(\mathrm{C}-5^{\prime}\right), 70.9\left(\mathrm{C}-2^{\prime}\right), 69.9\left(\mathrm{OCH}_{2} \mathrm{Ph}\right), 69.0\left(\mathrm{OCH}_{2} \mathrm{Ph}\right), 64.9\left(\mathrm{C}-4^{\prime}\right)$, $63.1(\mathrm{C}-6), 62.6\left(\mathrm{C}-5^{\prime \prime}\right), 61.0\left(\mathrm{C}-6^{\prime}\right), 55.5\left(\mathrm{OCH}_{3}\right), 20.7(2 \times \mathrm{C}(\mathrm{O})$ $\left.\mathrm{CH}_{3}\right)$; HRMS-ESI $m / z[\mathrm{M}+\mathrm{Na}]^{+}$calcd for $\mathrm{C}_{71} \mathrm{H}_{66} \mathrm{NaO}_{23}$ : 1309.3893. Found: 1309.3898 .

Methyl 5-O-Acetyl-2,3-O-xylylene- $\alpha$-D-xylofuranosyl- $(1 \rightarrow 4)-3-$ O-benzoyl-2,6-di-O-benzyl- $\alpha$-D-mannopyranoside (29). $R_{\mathrm{f}} 0.3(2: 1$, hexane-EtOAc); $[\alpha]_{\mathrm{D}}=+23.5\left(c=0.4, \mathrm{CHCl}_{3}\right) ;{ }^{1} \mathrm{H}$ NMR $(500$ $\left.\mathrm{MHz}^{\mathrm{CDCl}}\right)_{3} \delta_{\mathrm{H}} 8.14-8.08(\mathrm{~m}, 2 \mathrm{H}, \mathrm{ArH}), 7.70-7.64(\mathrm{~m}, 1 \mathrm{H}, \mathrm{ArH})$, 7.56-7.48 (m, 2H, ArH), 7.44-7.37 (m, 2H, ArH), 7.35-7.15 (m, $11 \mathrm{H}, \mathrm{ArH}), 6.55-6.48(\mathrm{~m}, 1 \mathrm{H}, \mathrm{ArH}), 5.63(\mathrm{dd}, 1 \mathrm{H}, J=3.3,9.8 \mathrm{~Hz}$, $\mathrm{H}-3), 5.23\left(\mathrm{~d}, 1 \mathrm{H}, J=5.1 \mathrm{~Hz}, \mathrm{H}-1^{\prime}\right), 4.84(\mathrm{~d}, 1 \mathrm{H}, J=1.8 \mathrm{~Hz}, \mathrm{H}-1)$, $4.74\left(\mathrm{~d}, 1 \mathrm{H}, J=11.9 \mathrm{~Hz}, \mathrm{OCH}_{2} \mathrm{Ph}\right), 4.71-4.62\left(\mathrm{~m}, 4 \mathrm{H}, \mathrm{OCH}_{2} \mathrm{Ph}\right)$, $4.61-4.55\left(\mathrm{~m}, 2 \mathrm{H}, \mathrm{OCH}_{2} \mathrm{Ph}\right), 4.28-4.07\left(\mathrm{~m}, 6 \mathrm{H}, \mathrm{H}-3^{\prime}, \mathrm{H}-4, \mathrm{H}-5, \mathrm{H}-\right.$ 6', H-6, OCH $\mathrm{O}_{2} \mathrm{Ph}$ ), 3.99 (ddd, $1 \mathrm{H}, J=1.8,9.9,6.0 \mathrm{~Hz}, \mathrm{H}-4^{\prime}$ ), 3.93 (dd, $1 \mathrm{H}, J=1.8,3.3 \mathrm{~Hz}, \mathrm{H}-2), 3.87$ (dd, $1 \mathrm{H}, J=10.8,1.8 \mathrm{~Hz}, \mathrm{H}-5^{\prime}$ ), $3.79\left(\mathrm{dd}, 1 \mathrm{H}, J=10.8,6.0 \mathrm{~Hz}, \mathrm{H}-5^{\prime}\right), 3.69($ app t, $1 \mathrm{H}, J=5.0 \mathrm{~Hz}, \mathrm{H}-$ $\left.2^{\prime}\right), 3.42\left(\mathrm{~s}, 3 \mathrm{H}, \mathrm{OCH}_{3}\right), 1.91\left(\mathrm{~s}, 3 \mathrm{H}, \mathrm{C}(\mathrm{O}) \mathrm{CH}_{3}\right) ;{ }^{13} \mathrm{C}$ NMR $(125$ $\left.\mathrm{MHz}, \mathrm{CDCl}_{3}\right) \delta_{\mathrm{C}} 170.7(\mathrm{C}=\mathrm{O}), 165.3(\mathrm{C}=\mathrm{O}), 138.6(\mathrm{Ar}), 137.9$ (Ar), $136.6(\mathrm{Ar}), 135.2(\mathrm{Ar}), 132.8(\mathrm{Ar}), 131.4(\mathrm{Ar}), 131.1(\mathrm{Ar})$, 130.6 (Ar), 129.7 (2C, Ar), 129.2 (Ar), 129.1 (Ar), 128.5 (2C, Ar), 128.23 (3C, Ar), 128.22 (3C, Ar), 127.7 (3C, Ar), 127.6 (3C, Ar), 127.4 (Ar), $103.3\left(\mathrm{C}-1^{\prime}\right), 98.8(\mathrm{C}-1), 81.4\left(\mathrm{C}-2^{\prime}\right), 80.3\left(\mathrm{C}-3^{\prime}, \mathrm{C}-4\right.$ or C-5), 76.10 (C-3', C-4 or C-5), 76.06 (C-2), 74.03 (C-3', C-4 or C5), 73.97 (C-3), $73.3\left(\mathrm{OCH}_{2} \mathrm{Ph}\right), 72.9\left(\mathrm{OCH}_{2} \mathrm{Ph}\right), 70.8\left(\mathrm{C}-4^{\prime}\right), 69.5$ $\left(\mathrm{OCH}_{2} \mathrm{Ph}\right), 69.3\left(\mathrm{OCH}_{2} \mathrm{Ph}\right), 69.1\left(\mathrm{C}-5^{\prime}\right), 63.5(\mathrm{C}-6), 54.8\left(\mathrm{OCH}_{3}\right)$, $20.8\left(\mathrm{C}(\mathrm{O}) \mathrm{CH}_{3}\right)$; HRMS-ESI $m / z[\mathrm{M}+\mathrm{Na}]^{+}$calcd for $\mathrm{C}_{43} \mathrm{H}_{46} \mathrm{NaO}_{12}$ : 777.2887. Found: 777.2880.

Octyl 5-O-Acetyl-2,3-O-xylylene- $\alpha$-D-xylofuranosyl-( $1 \rightarrow 3)-4,6-d i-$ o-benzoyl-2-deoxy-2-phthalimido- $\beta$-D-glucopyranoside (30). $R_{\mathrm{f}}$ $0.25\left(2: 1\right.$, hexane-EtOAc); $[\alpha]_{\mathrm{D}}=+69.0\left(c=0.3, \mathrm{CHCl}_{3}\right) ;{ }^{1} \mathrm{H}$ NMR $\left(500 \mathrm{MHz}, \mathrm{CDCl}_{3}\right) \delta_{\mathrm{H}} 8.11-8.05(\mathrm{~m}, 2 \mathrm{H}, \mathrm{ArH}), 7.95-7.86$ (m, 3H, ArH), 7.76-7.53 (m, 5H, ArH), 7.49-7.43 (m, 2H, ArH), 7.43-7.37 (m, 2H, ArH), 7.25-7.21 (m, 1H, ArH), 7.19-7.15 (m, $1 \mathrm{H}, \mathrm{ArH}), 7.13-7.08(\mathrm{~m}, 1 \mathrm{H}, \mathrm{ArH}), 6.53-6.49(\mathrm{~m}, 1 \mathrm{H}, \mathrm{ArH}), 6.15$ $($ app t, $1 \mathrm{H}, J=10.9 \mathrm{~Hz}, \mathrm{H}-3), 5.49(\mathrm{~d}, 1 \mathrm{H}, J=8.4 \mathrm{~Hz}, \mathrm{H}-1), 5.11(\mathrm{~d}$, $\left.1 \mathrm{H}, J=5.1 \mathrm{~Hz}, \mathrm{H}-1^{\prime}\right), 4.76\left(\mathrm{dd}, 1 \mathrm{H}, J=11.9,1.5 \mathrm{~Hz}, \mathrm{H}-5^{\prime}\right), 4.64(\mathrm{~d}$, $\left.1 \mathrm{H}, J=12.7 \mathrm{~Hz}, \mathrm{OCH}_{2} \mathrm{Ph}\right), 4.61-4.56\left(\mathrm{~m}, 2 \mathrm{H}, \mathrm{OCH}_{2} \mathrm{Ph}\right), 4.51(\mathrm{dd}$, $\left.1 \mathrm{H}, J=11.9,7.6 \mathrm{~Hz}, \mathrm{H}-5^{\prime}\right), 4.46\left(\mathrm{ddd}, 1 \mathrm{H}, J=1.5,7.6 \mathrm{~Hz}, \mathrm{H}-4^{\prime}\right)$, $4.38(\mathrm{dd}, 1 \mathrm{H}, J=8.4,10.9 \mathrm{~Hz}, \mathrm{H}-2), 4.26(\mathrm{dd}, 1 \mathrm{H}, J=5.3,7.7 \mathrm{~Hz}, \mathrm{H}-$ $\left.3^{\prime}\right)$, 4.18-4.12 (m, 1H, H-6'), 4.10-3.99 (m, 4H, H-3, H-4, H-6, $\left.\mathrm{OCH}_{2} \mathrm{Ph}\right), 3.82\left(\mathrm{dt}, 1 \mathrm{H}, J=9.8,6.2 \mathrm{~Hz}\right.$, octyl $\left.\mathrm{OCH}_{2}\right), 3.60($ app t, $\left.1 \mathrm{H}, J=5.3 \mathrm{~Hz}, \mathrm{H}-2^{\prime}\right), 3.45\left(\mathrm{~m}, 1 \mathrm{H}\right.$, octyl $\left.\mathrm{OCH}_{2}\right), 1.85(\mathrm{~s}, 3 \mathrm{H}$, $\left.\mathrm{C}(\mathrm{O}) \mathrm{CH}_{3}\right), 1.47-1.31(\mathrm{~m}, 2 \mathrm{H}$, octyl $), 1.18-1.10(\mathrm{~m}, 2 \mathrm{H}$, octyl $\left.\mathrm{CH}_{2}\right), 1.07-0.98\left(\mathrm{~m}, 6 \mathrm{H}\right.$, octyl $\left.\mathrm{CH}_{2}\right), 0.97-0.88\left(\mathrm{~m}, 2 \mathrm{H}\right.$, octyl $\left.\mathrm{CH}_{2}\right)$, 
$0.79\left(\mathrm{t}, 3 \mathrm{H}, J=7.3 \mathrm{~Hz}\right.$, octyl $\left.\mathrm{CH}_{3}\right) ;{ }^{13} \mathrm{C} \mathrm{NMR}\left(125 \mathrm{MHz} \mathrm{CDCl}_{3}\right) \delta_{\mathrm{C}}$ $170.9(\mathrm{C}=\mathrm{O}), 166.2(\mathrm{C}=\mathrm{O}), 165.4(\mathrm{C}=\mathrm{O}), 136.5(\mathrm{Ar}), 135.1$ (Ar), $134.0(\mathrm{Ar}), 133.9(\mathrm{Ar}), 133.0(\mathrm{Ar}), 132.9(\mathrm{Ar}), 131.4(\mathrm{Ar})$, 131.0 (Ar), 130.2 (Ar), 129.73 (2C, Ar), 129.71 (2C, Ar), 129.6 (Ar), 129.2 (2C, Ar), 129.1 (2C, Ar), 128.5 (2C, Ar), 128.4 (Ar), 123.5 (2C, Ar), $104.0\left(\mathrm{C}-1^{\prime}\right), 98.3(\mathrm{C}-1), 81.3\left(\mathrm{C}-2^{\prime}\right), 79.9\left(\mathrm{C}-3^{\prime}\right), 77.9$ (C4 or C-3), 76.7 (C-4'), 73.3 (C-5), 72.7 (C-4 or C-3), 70.2 (octyl $\left.\mathrm{OCH}_{2}\right), 69.2\left(\mathrm{OCH}_{2} \mathrm{Ph}\right), 69.1\left(\mathrm{OCH}_{2} \mathrm{Ph}\right), 63.5\left(\mathrm{C}-5^{\prime}\right), 63.1(\mathrm{C}-6)$, 55.1 (C-2), 31.6 (octyl $\mathrm{CH}_{2}$ ), 29.3 (octyl $\mathrm{CH}_{2}$ ), 29.14 (octyl $\mathrm{CH}_{2}$ ), $29.13\left(\right.$ octyl $\left.\mathrm{CH}_{2}\right), 25.8$ (octyl $\left.\mathrm{CH}_{2}\right), 22.6\left(\right.$ octyl $\left.\mathrm{CH}_{2}\right), 20.6(\mathrm{C}(\mathrm{O})$ $\mathrm{CH}_{3}$ ), 14.0 (octyl $\mathrm{CH}_{3}$ ); HRMS-ESI $m / z[\mathrm{M}+\mathrm{Na}]^{+}$calcd for $\mathrm{C}_{51} \mathrm{H}_{55} \mathrm{NO}_{14}$ : 905.3623 . Found: 905.3627.

5 -O-Acetyl-2,3-O-xylylene- $\alpha$ - $D$-xylofuranosyl- $(1 \rightarrow 3)-1,2: 5,6-d i-O-$ isopropylidene- $\alpha$-D-glucofuranose (31). $R_{\mathrm{f}} 0.3$ (2:1, hexaneEtOAc); $[\alpha]_{\mathrm{D}}=+35.7\left(c=0.5, \mathrm{CHCl}_{3}\right) ;{ }^{1} \mathrm{H}$ NMR $(500 \mathrm{MHz}$, $\left.\mathrm{CDCl}_{3}\right) \delta_{\mathrm{H}} 7.42-7.31(\mathrm{~m}, 4 \mathrm{H}, \mathrm{ArH}), 5.89(\mathrm{~d}, 1 \mathrm{H}, J=3.6 \mathrm{~Hz}, \mathrm{H}-1)$, $5.20\left(\mathrm{~d}, 1 \mathrm{H}, J=4.8 \mathrm{~Hz}, \mathrm{H}-1^{\prime}\right), 4.99\left(\mathrm{~d}, 1 \mathrm{H}, J=12.7 \mathrm{~Hz}, \mathrm{OCH}_{2} \mathrm{Ph}\right)$, $4.84\left(\mathrm{~d}, 1 \mathrm{H}, J=12.7 \mathrm{~Hz}, \mathrm{OCH}_{2} \mathrm{Ph}\right), 4.80(\mathrm{~d}, 1 \mathrm{H}, J=12.7 \mathrm{~Hz}$ $\left.\mathrm{OCH}_{2} \mathrm{Ph}\right), 4.75\left(\mathrm{~d}, 1 \mathrm{H}, J=12.7 \mathrm{~Hz}, \mathrm{OCH}_{2} \mathrm{Ph}\right), 4.60(\mathrm{~d}, 1 \mathrm{H}, J=3.6$ $\mathrm{Hz}, \mathrm{H}-2)$, 4.47-4.40 (m, 2H, H-4', H-5), 4.36 (dd, $1 \mathrm{H}, J=12.3,3.0$ $\left.\mathrm{Hz}, \mathrm{H}-5^{\prime}\right), 4.32\left(\mathrm{dd}, 1 \mathrm{H}, J=5.1,7.6 \mathrm{~Hz}, \mathrm{H}-3^{\prime}\right), 4.23-4.13(\mathrm{~m}, 4 \mathrm{H}, \mathrm{H}-$ 3, H-4, H-5', H-6'), 4.03 (app t, $1 \mathrm{H}, J=5.1 \mathrm{~Hz}, \mathrm{H}-2^{\prime}$ ), 3.95 (dd, $1 \mathrm{H}, J$ $=8.4,6.5 \mathrm{~Hz}, \mathrm{H}-6), 2.04\left(\mathrm{~s}, 3 \mathrm{H}, \mathrm{C}(\mathrm{O}) \mathrm{CH}_{3}\right), 1.51\left(\mathrm{~s}, 3 \mathrm{H}, \mathrm{CH}_{3}\right), 1.44$ $\left(\mathrm{s}, 3 \mathrm{H}, \mathrm{CH}_{3}\right), 1.39\left(\mathrm{~s}, 3 \mathrm{H}, \mathrm{CH}_{3}\right), 1.32\left(\mathrm{~s}, 3 \mathrm{H}, \mathrm{CH}_{3}\right) ;{ }^{13} \mathrm{C}$ NMR $(125$ $\left.\mathrm{MHz}, \mathrm{CDCl}_{3}\right) \delta_{\mathrm{C}} 170.8(\mathrm{C}=\mathrm{O}), 131.6(\mathrm{Ar}), 131.4(\mathrm{Ar}), 129.5(2 \mathrm{C}$, $\mathrm{Ar}), 111.8(\mathrm{Ar}), 108.8(\mathrm{Ar}), 105.2(\mathrm{C}-1), 102.1\left(\mathrm{C}-1^{\prime}\right), 83.9$ (C-2), 82.8 (C-2'), 81.28, 81.25 (C-3, C-4), 79.6 (C-3'), 76.3, 72.5 (C-4', C5), $69.6\left(\mathrm{OCH}_{2} \mathrm{Ph}\right), 69.0\left(\mathrm{OCH}_{2} \mathrm{Ph}\right), 67.2(\mathrm{C}-6), 63.5\left(\mathrm{C}-5^{\prime}\right), 26.9$ $\left(\mathrm{CH}_{3}\right), 26.7\left(\mathrm{CH}_{3}\right), 26.3\left(\mathrm{CH}_{3}\right), 25.5\left(\mathrm{CH}_{3}\right), 20.9\left(\mathrm{C}(\mathrm{O}) \mathrm{CH}_{3}\right)$. HRMS-ESI $m / z[\mathrm{M}+\mathrm{Na}]^{+}$calcd for $\mathrm{C}_{27} \mathrm{H}_{36} \mathrm{NaO}_{11}: 559.2155$. Found: 559.2164.

p-Methoxyphenyl 5-O-Acetyl-2,3-O-xylylene- $\alpha$-D-xylofuranosyl(1 $\rightarrow 4$ )-3-O-benzoyl-2,6-di-O-benzyl- $\alpha$-D-mannopyranoside (32). $R_{\mathrm{f}}$ 0.3 (2:1, hexane-EtOAc); ${ }^{1} \mathrm{H}$ NMR $\left(500 \mathrm{MHz}, \mathrm{CDCl}_{3}\right) \delta_{\mathrm{H}} 8.14-$ $8.07(\mathrm{~m}, 2 \mathrm{H}, \mathrm{ArH}), 7.70-7.64(\mathrm{~m}, 1 \mathrm{H}, \mathrm{ArH}), 7.56-7.43(\mathrm{~m}, 2 \mathrm{H}$, ArH), 7.34-7.26 (m, 5H, ArH), 7.25-7.00 (m, 10H, ArH), 6.84$6.75(\mathrm{~m}, 2 \mathrm{H}, \mathrm{ArH}), 6.50-6.44(\mathrm{~m}, 1 \mathrm{H}, \mathrm{ArH}), 5.82(\mathrm{dd}, 1 \mathrm{H}, J=3.3$, $9.8 \mathrm{~Hz}, \mathrm{H}-3), 5.49$ (d, $1 \mathrm{H}, J=1.8 \mathrm{~Hz}, \mathrm{H}-1), 5.23(\mathrm{~d}, 1 \mathrm{H}, J=5.1 \mathrm{~Hz}$, $\left.\mathrm{H}-1^{\prime}\right), 4.71-4.63\left(\mathrm{~m}, 2 \mathrm{H}, \mathrm{H}-4^{\prime}, \mathrm{OCH}_{2} \mathrm{Ph}\right), 4.63-4.55(\mathrm{~m}, 4 \mathrm{H}$, $\left.\mathrm{OCH}_{2} \mathrm{Ph}\right), 4.50\left(\mathrm{~d}, 1 \mathrm{H}, J=11.5 \mathrm{~Hz}, \mathrm{OCH}_{2} \mathrm{Ph}\right), 4.38-4.27(\mathrm{~m}, 2 \mathrm{H}$, H-4, H-5), 4.23-4.21 (m, 1H, H-3'), 4.16-4.04 (m, 3H, H-2, H-6', H-6), 3.85-3.73 (m, 5H, OCH $\left.3, \mathrm{H}-5^{\prime}, \mathrm{H}-5^{\prime}\right), 3.69$ (app t, $1 \mathrm{H}, J=5.1$ $\left.\mathrm{Hz}, \mathrm{H}-2^{\prime}\right), 1.87\left(\mathrm{~s}, 3 \mathrm{H}, \mathrm{C}(\mathrm{O}) \mathrm{CH}_{3}\right) ;{ }^{13} \mathrm{C} \mathrm{NMR}\left(125 \mathrm{MHz} \mathrm{CDCl}_{3}\right) \delta_{\mathrm{C}}$ $170.7(\mathrm{C}=\mathrm{O}), 165.5(\mathrm{C}=\mathrm{O}), 155.0(\mathrm{Ar}), 150.3(\mathrm{Ar}), 138.5(\mathrm{Ar})$, 137.87 (Ar), 136.6 (Ar), 135.1 (Ar), 132.9 (Ar), 131.3 (Ar), 131.1 (Ar), 130.5 (Ar), 129.6 (2C, Ar), $129.2(\mathrm{Ar}), 129.1$ (Ar), 128.6 (2C, $\mathrm{Ar}$ ), 128.23 (3C, Ar), 128.22 (3C, Ar), 127.7 (3C, Ar), 127.5 (3C, $\mathrm{Ar}), 127.4(\mathrm{Ar}), 103.6\left(\mathrm{C}-1^{\prime}\right), 97.0(\mathrm{C}-1), 81.5\left(\mathrm{C}-2^{\prime}\right), 80.3$ (C-3', C4 or C-5), 77.3 (C-3', C-4 or C-5), 76.0 (C-2), 74.2 (C-3', C-4 or C5), $73.7(\mathrm{C}-3), 73.2\left(\mathrm{OCH}_{2} \mathrm{Ph}\right), 71.3\left(\mathrm{OCH}_{2} \mathrm{Ph}\right), 70.83\left(\mathrm{C}-4^{\prime}\right), 69.51$ $\left(\mathrm{OCH}_{2} \mathrm{Ph}\right), 69.30\left(\mathrm{OCH}_{2} \mathrm{Ph}\right), 69.1\left({\mathrm{C}-5^{\prime}}^{\prime}\right), 63.5\left(\mathrm{C}^{\prime} 6^{\prime}\right), 55.6$ $\left(\mathrm{OCH}_{3}\right), 20.8\left(\mathrm{C}(\mathrm{O}) \mathrm{CH}_{3}\right)$; HRMS-ESI $\mathrm{m} / z[\mathrm{M}+\mathrm{Na}]^{+}$calcd for $\mathrm{C}_{49} \mathrm{H}_{50} \mathrm{NaO}_{13}: 869.3149$. Found: 869.3155.

Methyl 5-O-Acetyl-2,3-O-xylylene- $\alpha$-D-xylofuranosyl- $(1 \rightarrow 3)-2$ O-benzyl-4,6-O-benzylidene- $\alpha$-D-glucopyranoside (33 $\alpha$ ). $R_{f} 0.25$ $\left(2: 1\right.$, hexane-EtOAc); $[\alpha]_{\mathrm{D}}=-38.2\left(c=0.3, \mathrm{CHCl}_{3}\right) ;{ }^{1} \mathrm{H}$ NMR $\left(500 \mathrm{MHz}, \mathrm{CDCl}_{3}\right) \delta_{\mathrm{H}} 7.47-7.43(\mathrm{~m}, 2 \mathrm{H}, \mathrm{ArH}), 7.39-7.27(\mathrm{~m}, 12 \mathrm{H}$, $\mathrm{ArH}), 5.53\left(\mathrm{~s}, 1 \mathrm{H}, \mathrm{PhCHO}_{2}\right), 5.46\left(\mathrm{~d}, 1 \mathrm{H}, J=4.6 \mathrm{~Hz}, \mathrm{H}-1^{\prime}\right), 5.04$ (d, $\left.1 \mathrm{H}, J=12.7 \mathrm{~Hz}, \mathrm{OCH}_{2} \mathrm{Ph}\right), 4.87-4.78\left(\mathrm{~m}, 2 \mathrm{H}, \mathrm{OCH}_{2} \mathrm{Ph}\right), 4.73(\mathrm{~d}$, $\left.1 \mathrm{H}, J=12.7 \mathrm{~Hz}, \mathrm{OCH}_{2} \mathrm{Ph}\right), 4.71\left(\mathrm{~d}, 1 \mathrm{H}, J=11.7 \mathrm{~Hz}, \mathrm{OCH}_{2} \mathrm{Ph}\right)$, 4.61-4.54 (m, 3H, H-4', H-1, OCH $\left.\mathrm{OCh}_{2}\right), 4.39$ (app t, $J=9.4 \mathrm{~Hz}, \mathrm{H}-$ 3), 4.30-4.20 (m, 3H, H-3', H-5', H-6'), 4.10 (dd, $1 \mathrm{H}, J=12.2,7.1$ $\left.\mathrm{Hz}, \mathrm{H}-5^{\prime}\right), 4.03$ (app t, $\left.1 \mathrm{H}, J=4.9 \mathrm{~Hz}, \mathrm{H}-2^{\prime}\right), 3.80$ (app td, $1 \mathrm{H}, J=$ 9.8, $4.7 \mathrm{~Hz}, \mathrm{H}-5), 3.75-3.64(\mathrm{~m}, 2 \mathrm{H}, \mathrm{H}-4, \mathrm{H}-6), 3.56(\mathrm{dd}, 1 \mathrm{H}, J=$ 3.7, $9.5 \mathrm{~Hz}, \mathrm{H}-2), 3.37\left(\mathrm{~s}, 3 \mathrm{H}, \mathrm{OCH}_{3}\right), 1.95\left(\mathrm{~s}, 3 \mathrm{H}, \mathrm{C}(\mathrm{O}) \mathrm{CH}_{3}\right) ;{ }^{13} \mathrm{C}$ NMR $\left(125 \mathrm{MHz}, \mathrm{CDCl}_{3}\right) \delta_{\mathrm{C}} 170.9(\mathrm{C}=\mathrm{O}), 138.1(\mathrm{Ar}), 137.4(\mathrm{Ar})$, $136.6(\mathrm{Ar}), 135.9(\mathrm{Ar}), 131.6(\mathrm{Ar}), 131.3(\mathrm{Ar}), 129.4(\mathrm{Ar}), 129.3$ (Ar), 128.9 (2C, Ar), 128.4 (2C, Ar), 128.2 (2C, Ar), 128.0 (2C, Ar), 127.9 (Ar), 126.1 (2C, Ar), $101.3\left(\mathrm{PhCHO}_{2}\right), 100.2\left(\mathrm{C}-1^{\prime}\right), 99.0$ (C1), 83.1 (C-2'), 82.5 (C-4), 79.9 (C-3'), 77.4 (C-2), 75.7 (C-4'), 73.3
$\left(\mathrm{OCH}_{2} \mathrm{Ph}\right), 72.35(\mathrm{C}-3), 70.1\left(\mathrm{OCH}_{2} \mathrm{Ph}\right), 69.1\left(\mathrm{OCH}_{2} \mathrm{Ph}\right), 68.9$ (C6), $63.2\left(\mathrm{C}-5^{\prime}\right), 62.0(\mathrm{C}-5), 55.2\left(\mathrm{OCH}_{3}\right), 20.9\left(\mathrm{C}(\mathrm{O}) \mathrm{CH}_{3}\right)$; HRMSESI $m / z[\mathrm{M}+\mathrm{Na}]^{+}$calcd for $\mathrm{C}_{36} \mathrm{H}_{40} \mathrm{NaO}_{11}$ : 671.2468. Found: 671.2474 .

Methyl 5-O-Acetyl-2,3-O-xylylene- $\beta$-D-xylofuranosyl- $(1 \rightarrow 3)-2-0$ -

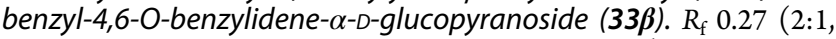
hexane-EtOAc); $[\alpha]_{\mathrm{D}}=+20.3\left(c=0.2, \mathrm{CHCl}_{3}\right) ;{ }^{1} \mathrm{H}$ NMR $(500$ $\left.\mathrm{MHz}, \mathrm{CDCl}_{3}\right) \delta_{\mathrm{H}} 7.53-7.48(\mathrm{~m}, 2 \mathrm{H}, \mathrm{ArH}), 7.42-7.27(\mathrm{~m}, 12 \mathrm{H}$, $\mathrm{ArH}), 5.48\left(\mathrm{~s}, 1 \mathrm{H}, \mathrm{PhCHO}_{2}\right), 5.34\left(\mathrm{~d}, 1 \mathrm{H}, J=2.7 \mathrm{~Hz}, \mathrm{H}-1^{\prime}\right), 4.89-$ $4.78\left(\mathrm{~m}, 3 \mathrm{H}, \mathrm{OCH}_{2} \mathrm{Ph}\right), 4.74-4.67\left(\mathrm{~m}, 2 \mathrm{H}, \mathrm{OCH}_{2} \mathrm{Ph}\right), 4.63-4.57$ $\left(\mathrm{m}, 2 \mathrm{H}, \mathrm{H}-1, \mathrm{OCH}_{2} \mathrm{Ph}\right), 4.41\left(\mathrm{dd}, 1 \mathrm{H}, J=11.9,3.8 \mathrm{~Hz}, \mathrm{H}-5^{\prime}\right), 4.29$ $\left(\mathrm{ddd}, 1 \mathrm{H}, J=7.0,3.8,7.0 \mathrm{~Hz}, \mathrm{H}-4^{\prime}\right), 4.24(\mathrm{dd}, 1 \mathrm{H}, J=4.7,10.1 \mathrm{~Hz}$, H-6'), 4.18-4.07 (m, 4H, H-2', H-3', H-5', H-3), 3.79 (app td, 1H, J = 9.8, $4.7 \mathrm{~Hz}, \mathrm{H}-5), 4.24($ app t, $1 \mathrm{H}, J=10.3 \mathrm{~Hz}, \mathrm{H}-6), 3.53-3.46(\mathrm{~m}$, $2 \mathrm{H}, \mathrm{H}-2, \mathrm{H}-4), 3.38\left(\mathrm{~s}, 3 \mathrm{H}, \mathrm{OCH}_{3}\right), 1.86\left(\mathrm{~s}, 3 \mathrm{H}, \mathrm{C}(\mathrm{O}) \mathrm{CH}_{3}\right) ;{ }^{13} \mathrm{C}$ NMR $\left(125 \mathrm{MHz}, \mathrm{CDCl}_{3}\right) \delta_{\mathrm{C}} 170.7(\mathrm{C}=\mathrm{O}), 138.2(\mathrm{Ar}), 137.4(\mathrm{Ar})$, 136.5 (Ar), $135.7(\mathrm{Ar}), 131.7(\mathrm{Ar}), 131.3(\mathrm{Ar}), 129.6(\mathrm{Ar}), 129.5$ (Ar), 128.7 (Ar), 128.4 (2C, Ar), 128.2 (2C, Ar), 128.0 (2C, Ar),

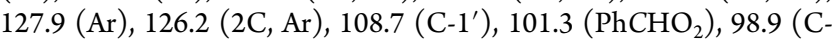
1), 87.1 (C-2' or C-3'), 81.1 (C-2' or C-3'), 80.6 (C-2 or C-4), 79.0 (C-2 or C-4), $78.4\left(\mathrm{C}-4^{\prime}\right), 76.5(\mathrm{C}-3), 73.5\left(\mathrm{OCH}_{2} \mathrm{Ph}\right), 69.2(2 \mathrm{x}$ $\left.\mathrm{OCH}_{2} \mathrm{Ph}\right), 69.0(\mathrm{C}-6), 64.3\left(\mathrm{C}-5^{\prime}\right), 62.3(\mathrm{C}-5), 55.3\left(\mathrm{OCH}_{3}\right), 20.9$ $\left(\mathrm{C}(\mathrm{O}) \mathrm{CH}_{3}\right)$; HRMS-ESI $\mathrm{m} / z[\mathrm{M}+\mathrm{Na}]^{+}$calcd for $\mathrm{C}_{36} \mathrm{H}_{40} \mathrm{NaO}_{11}$ : 671.2468. Found: 671.2481 .

8-Aminooctyl 5-Deoxy-5-methylthio- $\alpha$-D-xylofuranosyl-(1 $\rightarrow 4)$ $\alpha$-D-mannopyranosyl- $(1 \rightarrow 5)-\beta$-D-arabinofuranosyl- $(1 \rightarrow 2)-\alpha$-D-arabinofuranosyl- $(1 \rightarrow 5)$ - $\alpha$-D-arabinofuranosyl- $(1 \rightarrow 5)$ - $\alpha$-D-arabinofuranoside (36). To a solution of liquid $\mathrm{NH}_{3}(25 \mathrm{~mL})$ at $-78{ }^{\circ} \mathrm{C}$ was added sodium metal $(50 \mathrm{mg})$ until a deep blue solution was produced. A solution of $47(37 \mathrm{mg}, 0.02 \mathrm{mmol})$ in THF $(0.5 \mathrm{~mL})$ was then added over a period of $1 \mathrm{~min}$, making sure that the deep blue color persisted. The reaction mixture was stirred at $-78{ }^{\circ} \mathrm{C}$ for $2 \mathrm{~h}$, and $\mathrm{CH}_{3} \mathrm{OH}$ was then added until the dark blue color disappeared and the solution became clear. The solution was then warmed to rt by blowing air gently over the solution, which also helped evaporate the $\mathrm{NH}_{3}$. When the reaction mixture reached $\mathrm{rt}, \mathrm{CH}_{3} \mathrm{OH}(8 \mathrm{~mL})$ was added, and the $\mathrm{pH}$ of the solution was brought to $\sim 8$ by the careful addition of Amberlite IR $120 \mathrm{H}^{+}$resin. The solution was filtered to remove the resin, and the filtrate was concentrated. The residue was purified by chromatography $\left(6: 2: 1 \quad \mathrm{CH}_{3} \mathrm{OH}-\mathrm{NH}_{4} \mathrm{OH}-\mathrm{H}_{2} \mathrm{O}\right)$ on Iatrobeads $6 \mathrm{RS}-8060$ to give $36(14.5 \mathrm{mg}, 74 \%)$ as a thick syrup that was later lyophilized from water to give a fluffy solid. $R_{\mathrm{f}}=0.43(5: 2: 1$ $\left.\mathrm{CH}_{3} \mathrm{OH}-\mathrm{NH}_{4} \mathrm{OH}-\mathrm{H}_{2} \mathrm{O}\right) ;[\alpha]_{\mathrm{D}}+72.6\left(c=0.2, \mathrm{CH}_{3} \mathrm{OH}\right) ;{ }^{1} \mathrm{H}$ NMR $\left(700 \mathrm{MHz}, \mathrm{CD}_{3} \mathrm{OD}\right) \delta_{\mathrm{H}} 5.37(\mathrm{~d}, 1 \mathrm{H}, J=4.2 \mathrm{~Hz}, \mathrm{H}-1), 5.08(\mathrm{~d}, 1 \mathrm{H}, J$ $=1.8 \mathrm{~Hz}, \mathrm{H}-1), 5.03(\mathrm{~d}, 1 \mathrm{H}, J=4.8 \mathrm{~Hz}, \mathrm{H}-1), 4.95(\mathrm{~d}, 1 \mathrm{H}, J=1.2 \mathrm{~Hz}$, $\mathrm{H}-1), 4.84(\mathrm{~d}, 1 \mathrm{H}, J=1.2 \mathrm{~Hz}, \mathrm{H}-1), 4.32(\mathrm{dt}, 1 \mathrm{H}, J=7.2,5.4 \mathrm{~Hz})$, 4.15-4.10 (m, 2H), 4.09-3.77 (m, 26H), 3.72-3.64 (m, 7H), 3.42 $(\mathrm{dt}, 1 \mathrm{H}, J=9.6,6.5 \mathrm{~Hz}), 3.07-3.02(\mathrm{~m}, 1 \mathrm{H}), 2.76-2.71(\mathrm{~m}, 1 \mathrm{H})$, $2.63-2.58(\mathrm{~m}, 2 \mathrm{H}), 2.16(\mathrm{~s}, 3 \mathrm{H}), 1.59-1.42(\mathrm{~m}, 4 \mathrm{H}), 1.41-1.30(\mathrm{~m}$, $8 \mathrm{H}) ;{ }^{13} \mathrm{C}$ NMR $\left(125 \mathrm{MHz}, \mathrm{CD}_{3} \mathrm{OD}\right) \delta_{\mathrm{C}} 109.7(\mathrm{C}-1), 109.5(\mathrm{C}-1)$, 107.4 (C-1), 104.8 (C-1), 102.4 (C-1), 101.7 (C-1), 89.5, 84.4, 84.0, 83.7, 83.5, 83.3, 82.3, 80.5, 79.2, 79.0, 78.9, 78.4, 77.2, 76.7, 76.1, 76.0, 73.5, 72.3, 72.2, 69.8, 68.9, 68.2, 68.2, 62.9, 62.4, 42.6 $\left(\mathrm{CH}_{2} \mathrm{NH}_{2}\right.$ ), 34.5, 34.0, 30.7 (octyl $\mathrm{CH}_{2}$ ), 30.6 (octyl $\mathrm{CH}_{2}$ ), 30.4 (octyl $\mathrm{CH}_{2}$ ), 28.0 (octyl $\mathrm{CH}_{2}$ ), 27.2 (octyl $\left.\mathrm{CH}_{2}\right), 16.3\left(\mathrm{SCH}_{3}\right)$. HRMS-ESI $m / z[\mathrm{M}+\mathrm{H}]^{+}$calcd for $\mathrm{C}_{40} \mathrm{H}_{72} \mathrm{NO}_{25} \mathrm{~S}$ : 998.4109. Found: 998.4134.

p-Tolyl 5-O-Methanesulfonyl-1-thio-2,3-O-xylylene- $\alpha$-D-xylofuranoside (37). To a solution of $12(175 \mathrm{mg}, 0.3 \mathrm{mmol})$ in pyridine $(10 \mathrm{~mL})$ was added methanesulfonyl chloride $(47 \mu \mathrm{L}, 0.6 \mathrm{mmol})$ at 0 ${ }^{\circ} \mathrm{C}$. The reaction mixture was stirred at $\mathrm{rt}$ for $2 \mathrm{~h}$ and then concentrated to a syrup that was purified by flash column chromatography $\left(2: 1: 1\right.$, hexanes-EtOAc- $\left.\mathrm{CH}_{2} \mathrm{Cl}_{2}\right)$ to give 37 (129 $\mathrm{mg}, 97 \%)$ as a solid: $R_{\mathrm{f}} 0.2\left(3: 1\right.$, hexanes-EtOAc); $[\alpha]_{\mathrm{D}}=-121.9(c$ $\left.=0.4, \mathrm{CHCl}_{3}\right) ;{ }^{1} \mathrm{H} \mathrm{NMR}\left(700 \mathrm{MHz}, \mathrm{CDCl}_{3}\right) \delta_{\mathrm{H}} 7.43-7.40(\mathrm{~m}, 2 \mathrm{H}$, $\mathrm{Ar}), 7.37-7.34(\mathrm{~m}, 3 \mathrm{H}, \mathrm{Ar}), 7.33-7.30(\mathrm{~m}, 1 \mathrm{H}, \mathrm{Ar}), 7.08$ (d, $J=7.9$ $\mathrm{Hz}, 2 \mathrm{H}, \mathrm{Ar}), 5.03(\mathrm{~d}, 1 \mathrm{H}, J=7.6 \mathrm{~Hz}, \mathrm{H}-1), 4.94(\mathrm{~d}, 1 \mathrm{H}, J=12.8 \mathrm{~Hz}$, $\left.\mathrm{OCH}_{2} \mathrm{Ph}\right), 4.88\left(\mathrm{~d}, 1 \mathrm{H}, J=12.6 \mathrm{~Hz}, \mathrm{OCH}_{2} \mathrm{Ph}\right), 4.81(\mathrm{~d}, 1 \mathrm{H}, J=12.8$ $\mathrm{Hz}, \mathrm{OCH}_{2} \mathrm{Ph}$ ), $4.71\left(\mathrm{~d}, 1 \mathrm{H}, J=12.6 \mathrm{~Hz}, \mathrm{OCH}_{2} \mathrm{Ph}, \mathrm{OCH}_{2} \mathrm{Ph}\right), 4.40-$ 4.35 (m, 3H, H-5, H-4), 4.25-4.20 (m, 1H, H-3), 3.95 (dd, 1H, $J=$ 
7.6, 5.4 Hz, H-2), 3.02 (s, 3H, S $\left.(\mathrm{O})_{2} \mathrm{CH}_{3}\right), 2.31$ (s, 3H, $\left.\mathrm{ArCH}_{3}\right) .{ }^{13} \mathrm{C}$ NMR $\left(175 \mathrm{MHz}, \mathrm{CDCl}_{3}\right) \delta_{\mathrm{C}} 138.0(\mathrm{Ar}), 136.0(\mathrm{Ar}), 135.1(\mathrm{Ar})$, 132.4 (Ar), 131.7 (Ar), 131.5 (Ar), 129.9 (Ar), 129.8 (Ar), 129.7 (Ar), 129.3 (Ar), 88.7 (C-1), 84.9 (C-2), 80.4 (C-3), 78.1 (C-4), 69.9 $\left(\mathrm{OCH}_{2}\right), 69.1(\mathrm{C}-5), 68.7\left(\mathrm{OCH}_{2}\right), 37.6\left(\mathrm{~S}(\mathrm{O})_{2} \mathrm{CH}_{3}\right), 21.1\left(\mathrm{ArCH}_{3}\right)$. HRMS-ESI $m / z[\mathrm{M}+\mathrm{Na}]^{+}$calcd for $\mathrm{C}_{21} \mathrm{H}_{24} \mathrm{NaO}_{6} \mathrm{~S}_{2}: 459.0907$. Found: 459.0906.

p-Tolyl 6-O-Benzyl-2,3-di-O-benzoyl-4-O-levulinyl-1-thio- $\alpha$-Dmannopyranoside (38). Thioglycoside $41(0.9 \mathrm{~g}, 1.54 \mathrm{mmol})$, levulinic acid $(0.21 \mathrm{~g}, 1.84 \mathrm{mmol})$, and DMAP $(0.01 \mathrm{~g}, 0.08 \mathrm{mmol})$ were dissolved in $\mathrm{CH}_{2} \mathrm{Cl}_{2}(40 \mathrm{~mL})$. The solution was cooled to $0{ }^{\circ} \mathrm{C}$ before EDCI $(0.42 \mathrm{~g}, 2.3 \mathrm{mmol})$ was added. After stirring at $\mathrm{rt}$ for $2 \mathrm{~h}$, water was added, and the organic layer was subsequently dried with anhydrous $\mathrm{Na}_{2} \mathrm{SO}_{4}$ and filtered, and the filtrate was concentrated. The residue was purified by chromatography $(4: 1$, hexanes-EtOAc) to give $38(0.97 \mathrm{mg}, 92 \%)$ as an oil. $R_{\mathrm{f}} 0.2\left(4: 1\right.$, hexanes-EtOAc); $[\alpha]_{\mathrm{D}}$ $=-6.2\left(c=0.3, \mathrm{CHCl}_{3}\right) ;{ }^{1} \mathrm{H} \mathrm{NMR}\left(700 \mathrm{MHz}, \mathrm{CDCl}_{3}\right) \delta_{\mathrm{H}} 8.01(\mathrm{dd}$, $2 \mathrm{H}, J=8.4,1.3 \mathrm{~Hz}, \mathrm{Ar}), 7.91$ (dd, $2 \mathrm{H}, J=8.4,1.3 \mathrm{~Hz}, \mathrm{Ar}$ ), 7.55 (app $\mathrm{tt}, 1 \mathrm{H}, J=7.6,1.3 \mathrm{~Hz}, \mathrm{Ar}), 7.53-7.49(\mathrm{~m}, 1 \mathrm{H}, \mathrm{Ar}), 7.48-7.43(\mathrm{~m}$, $2 \mathrm{H}, \mathrm{Ar}), 7.39-7.33(\mathrm{~m}, 8 \mathrm{H}, \mathrm{Ar}), 7.32-7.28(\mathrm{~m}, 1 \mathrm{H}, \mathrm{Ar}), 7.07(\mathrm{~d}, 2 \mathrm{H}$, $J=7.9 \mathrm{~Hz}, \mathrm{Ar}), 5.88(\mathrm{dd}, 1 \mathrm{H}, J=3.3,1.7 \mathrm{~Hz}, \mathrm{H}-2), 5.84($ app t, $1 \mathrm{H}, J$ $=9.8 \mathrm{~Hz}, \mathrm{H}-4), 5.68(\mathrm{dd}, 1 \mathrm{H}, J=10.0,3.3 \mathrm{~Hz}, \mathrm{H}-3), 5.64(\mathrm{~d}, 1 \mathrm{H}, J=$ $1.5 \mathrm{~Hz}, \mathrm{H}-1)$, 4.73-4.67 (m, $1 \mathrm{H}, \mathrm{H}-5), 4.62(\mathrm{~d}, 1 \mathrm{H}, J=11.5 \mathrm{~Hz}$, $\left.\mathrm{OCH}_{2} \mathrm{Ph}\right), 4.55\left(\mathrm{~d}, 1 \mathrm{H}, J=11.5 \mathrm{~Hz}, \mathrm{OCH}_{2} \mathrm{Ph}\right), 3.81-3.74(\mathrm{~m}, 2 \mathrm{H}$, $\mathrm{H}-6)$, 2.67-2.38 (m, 4H, OC $\left.(\mathrm{O}) \mathrm{CH}_{2} \mathrm{CH}_{2} \mathrm{C}(\mathrm{O})\right), 2.31(\mathrm{~s}, 3 \mathrm{H}$, $\left.\mathrm{C}(\mathrm{O}) \mathrm{CH}_{3}\right), 2.05\left(\mathrm{~s}, 3 \mathrm{H}, \mathrm{ArCH}_{3}\right) \cdot{ }^{13} \mathrm{C}$ NMR $\left(175 \mathrm{MHz}, \mathrm{CDCl}_{3}\right) \delta_{\mathrm{C}}$ $205.8(\mathrm{C}=\mathrm{O}), 171.7(\mathrm{C}=\mathrm{O}), 165.4(\mathrm{C}=\mathrm{O}), 165.3(\mathrm{C}=\mathrm{O}), 138.2$ (Ar), $138.2(\mathrm{Ar}), 133.4(\mathrm{Ar}), 133.3$ (Ar), 132.7 (Ar), 129.9 (2C, Ar), 129.8 (Ar), $129.3(\mathrm{Ar}), 129.2(\mathrm{Ar}), 129.1$ (Ar), 128.5 (Ar), 128.4 (Ar), $128.2(\mathrm{Ar}), 127.8(\mathrm{Ar}), 127.5(\mathrm{Ar}), 86.2(\mathrm{C}-1), 73.6\left(\mathrm{OCH}_{2} \mathrm{Ph}\right)$, 71.9 (C-2), 70.9 (C-5), 70.6 (C-3), 68.9 (C-6), 66.9 (C-4), 37.8 $\left(\mathrm{OC}(\mathrm{O}) \mathrm{CH}_{2} \mathrm{CH}_{2} \mathrm{C}(\mathrm{O})\right), 29.55\left(\mathrm{C}(\mathrm{O}) \mathrm{CH}_{3}\right), 27.9((\mathrm{OC}(\mathrm{O})$ $\left.\mathrm{CH}_{2} \mathrm{CH}_{2} \mathrm{C}(\mathrm{O})\right)$, $21.1\left(\mathrm{ArCH}_{3}\right)$. HRMS-ESI $m / z\left[\mathrm{M}+\mathrm{NH}_{4}\right]^{+}$calcd for $\mathrm{C}_{39} \mathrm{H}_{42} \mathrm{NO}_{9} \mathrm{~S}$ : 700.2575 . Found: 700.2579 .

p-Tolyl 6-O-Benzyl-2,3-di-O-benzoyl-1-thio- $\alpha$-D-mannopyranoside (41). To a solution of $\mathbf{4 0}^{27}(0.58 \mathrm{~g}, 1.0 \mathrm{mmol})$ in $\mathrm{CH}_{2} \mathrm{Cl}_{2}(30$ $\mathrm{mL})$ were added triethylsilane $(0.8 \mathrm{~mL}, 5.0 \mathrm{mmol})$, TFA $(0.38 \mathrm{~mL}$, $5.0 \mathrm{~mL})$, and TFAA $(0.21 \mathrm{~mL}, 1.5 \mathrm{mmol})$ at $0{ }^{\circ} \mathrm{C}$. The mixture was stirred overnight allowing the temperature warm to rt. After completion of the reaction (TLC), the reaction mixture was cooled to $0{ }^{\circ} \mathrm{C}$ before the addition of $\mathrm{CH}_{3} \mathrm{OH}$. The solution was then neutralized by the careful addition of saturated aq. $\mathrm{NaHCO}_{3}$, and the organic layer was separated and washed with brine. The organic layer was subsequently dried with anhydrous $\mathrm{Na}_{2} \mathrm{SO}_{4}$ and filtered, and the filtrate was concentrated. The residue was purified by chromatography (8:1, hexanes-EtOAc) to give $\mathbf{4 1}(0.54 \mathrm{~g}, 92 \%)$ as an oil: $R_{\mathrm{f}} 0.4(4: 1$, hexanes-EtOAc); $[\alpha]_{\mathrm{D}}=+33.9\left(c=0.3, \mathrm{CHCl}_{3}\right) ;{ }^{1} \mathrm{H}$ NMR $(700$ $\left.\mathrm{MHz}, \mathrm{CDCl}_{3}\right) \delta_{\mathrm{H}} 8.05(\mathrm{dd}, 2 \mathrm{H}, J=8.4,1.4 \mathrm{~Hz}, \mathrm{Ar}), 7.94(\mathrm{dd}, 2 \mathrm{H}, J=$ 8.2, 1.4 Hz, Ar), 7.61-7.56 (m, 1H, Ar), 7.54-7.49 (m, 1H, Ar), 7.47-7.39 (m, 4H, Ar), 7.38-7.33 (m, 6H, Ar), 7.33-7.28 (m, $1 \mathrm{H}$, Ar), 7.11-7.07 (m, 2H, Ar), $5.86(\mathrm{dd}, 1 \mathrm{H}, J=3.3,1.6 \mathrm{~Hz}, \mathrm{H}-2), 5.62$ $(\mathrm{d}, 1 \mathrm{H}, J=1.6 \mathrm{~Hz}, \mathrm{H}-1), 5.59$ (dd, $1 \mathrm{H}, J=9.8,3.3 \mathrm{~Hz}, \mathrm{H}-3), 4.70$ (d, $\left.1 \mathrm{H}, J=11.8 \mathrm{~Hz}, \mathrm{OCH}_{2} \mathrm{Ph}\right), 4.60\left(\mathrm{~d}, 1 \mathrm{H}, J=11.8 \mathrm{~Hz}, \mathrm{OCH}_{2} \mathrm{Ph}\right), 4.55$ (app dt, $1 \mathrm{H}, J=9.7,4.1 \mathrm{~Hz}, \mathrm{H}-5), 4.45($ app t, $1 \mathrm{H}, J=9.7 \mathrm{~Hz}, \mathrm{H}-4)$, $3.99(\mathrm{dd}, 1 \mathrm{H}, J=10.6,4.6 \mathrm{~Hz}, \mathrm{H}-6 \mathrm{a}), 3.90(\mathrm{dd}, 1 \mathrm{H}, J=10.6,3.6 \mathrm{~Hz}$, $\mathrm{H}-6 \mathrm{~b}), 2.88(\mathrm{~s}, 1 \mathrm{H}, \mathrm{OH}), 2.32\left(\mathrm{~s}, 3 \mathrm{H}, \mathrm{ArCH}_{3}\right) .{ }^{13} \mathrm{C} \mathrm{NMR}(175 \mathrm{MHz}$, $\left.\mathrm{CDCl}_{3}\right) \delta_{\mathrm{C}} 206.6(\mathrm{C}=\mathrm{O}), 166.5(\mathrm{C}=\mathrm{O}), 165.3(\mathrm{C}=\mathrm{O}), 138.1$ (Ar), 138.0 (Ar), 133.4 (Ar), 133.3 (Ar), 132.7 (Ar), 129.9 (Ar), 129.8 (2C, Ar), 129.5 (Ar), 129.4 (Ar), 129.3 (Ar), 128.5 (Ar), 128.4 (Ar), 127.6(2C, Ar), $86.4\left(\mathrm{OCH}_{2} \mathrm{Ph}\right), 73.7$ (C-1), 73.1 (C-3), 72.5 (C-2), 72.0 (C-5), 69.9 (C-6), 67.7 (C-4), $21.1\left(\mathrm{ArCH}_{3}\right)$. HRMS-ESI $m / z[\mathrm{M}+\mathrm{Na}]^{+}$calcd for $\mathrm{C}_{34} \mathrm{H}_{32} \mathrm{NaO}_{7} \mathrm{~S}: 607.1761$. Found: 607.1759.

8-Azidooctyl 5-O-p-Methoxybenzyl-2,3-O-xylylene- $\beta$-D-arabinofuranosyl-( $1 \rightarrow 2)$-3,5-di-O-benzyl- $\alpha$-D-arabinofuranosyl- $(1 \rightarrow 5)-2,3-$ di-O-benzyl- $\alpha$-D-arabinofuranosyl- $(1 \rightarrow 5)-2,3-d i-O-b e n z y l-\alpha$ - D-arabinofuranoside (42). To a mixture of donor $6^{14}(0.59 \mathrm{~g}, 1.2 \mathrm{mmol})$ and acceptor $39^{19}(1.1 \mathrm{~g}, 1.0 \mathrm{mmol})$ in $\mathrm{CH}_{2} \mathrm{Cl}_{2}(80 \mathrm{~mL})$ were added $4 \AA$ molecular sieves $(0.3 \mathrm{~g})$ at rt. After stirring for $1 \mathrm{~h}$ and then cooling to $-60{ }^{\circ} \mathrm{C}$, NIS $(146 \mathrm{mg}, 0.65 \mathrm{mmol})$ and TMSOTf $(9 \mu \mathrm{L}$, $0.05 \mathrm{mmol}$ ) were added to the mixture, and the reaction was monitored by TLC. After stirring for $1 \mathrm{~h}$ at $-40{ }^{\circ} \mathrm{C}$, the reaction was quenched by the addition of $\mathrm{Et}_{3} \mathrm{~N}$. The solution was diluted with $\mathrm{CH}_{2} \mathrm{Cl}_{2}$ and filtered through Celite. The filtrate was then washed with saturated aq. $\mathrm{Na}_{2} \mathrm{~S}_{2} \mathrm{O}_{3}$ and brine. The organic layer was subsequently dried with anhydrous $\mathrm{Na}_{2} \mathrm{SO}_{4}$, filtered, and concentrated, and the residue was purified by chromatography $(5: 1$, hexane-EtOAc) to give $42(1.16 \mathrm{~g}, 80 \%)$ as a colorless oil: $R_{\mathrm{f}} 0.3(3: 1$, hexanes-EtOAc); $[\alpha]_{\mathrm{D}}=+39.5\left(c=0.1, \mathrm{CHCl}_{3}\right) ;{ }^{1} \mathrm{H} \mathrm{NMR}\left(600 \mathrm{MHz}, \mathrm{CDCl}_{3}\right) \delta_{\mathrm{H}} 7.28$ $(\mathrm{dt}, 2 \mathrm{H}, J=6.0,2.5 \mathrm{~Hz}, \mathrm{Ar}), 7.24-7.11(\mathrm{~m}, 33 \mathrm{H}, \mathrm{Ar}), 7.03(\mathrm{~d}, 2 \mathrm{H}, J=$ $8.6 \mathrm{~Hz}, \mathrm{Ar}), 6.71-6.68(\mathrm{~m}, 2 \mathrm{H}, \mathrm{Ar}), 5.05\left(\mathrm{~s}, 1 \mathrm{H}, \mathrm{H}-1^{\prime \prime}\right), 5.04(\mathrm{~s}, 1 \mathrm{H}$, $\left.\mathrm{H}-1^{\prime}\right), 4.96\left(\mathrm{~d}, 1 \mathrm{H}, J=4.8 \mathrm{~Hz}, \mathrm{H}-1^{\prime \prime \prime}\right), 4.92(\mathrm{~s}, 1 \mathrm{H}, \mathrm{H}-1), 4.86(\mathrm{~d}, 1 \mathrm{H}$, $\left.J=12.8 \mathrm{~Hz}, \mathrm{OCH}_{2} \mathrm{Ph}\right), 4.75-4.64\left(\mathrm{~m}, 3 \mathrm{H}, \mathrm{OCH}_{2} \mathrm{Ph}\right), 4.53(\mathrm{~d}, 1 \mathrm{H}, J$ $\left.=11.8 \mathrm{~Hz}, \mathrm{OCH}_{2} \mathrm{Ph}\right), 4.49-4.38\left(\mathrm{~m}, 9 \mathrm{H}, \mathrm{OCH}_{2} \mathrm{Ph}\right), 4.35-4.28(\mathrm{~m}$, $\left.3 \mathrm{H}, \mathrm{OCH}_{2} \mathrm{Ph}\right), 4.25-4.20\left(\mathrm{~m}, 2 \mathrm{H}, \mathrm{OCH}_{2} \mathrm{Ph}, \mathrm{H}-2^{\prime \prime}\right), 4.11-4.06(\mathrm{~m}$, $\left.3 \mathrm{H}, \mathrm{H}-4, \mathrm{H}-4^{\prime}, \mathrm{H}-4^{\prime \prime}\right), 4.00-3.94$ (m, 5H, H-3"', H-3", H-3', H-3, H$\left.2^{\prime}\right), 3.94(\mathrm{dd}, 1 \mathrm{H}, J=3.5,1.3 \mathrm{~Hz}, \mathrm{H}-2), 3.92-3.86$ (m, $2 \mathrm{H}, \mathrm{H}-4^{\prime \prime \prime}, \mathrm{H}-$ $\left.2^{\prime \prime \prime}\right)$, 3.81-3.77 (m, 2H, H-5a, H-5'a), 3.66 (s, 3H, $\left.\mathrm{OCH}_{3}\right), 3.63-$ 3.57 (m, 3H, H-5b, H-5'b, octyl $\mathrm{OCH}_{2}$ ), 3.53-3.44 (m, 3H, H-5'" a, H-5"'b, H-5"a), 3.40 (dd, 1H, J = 9.9, $5.5 \mathrm{~Hz}, \mathrm{H}-5^{\prime \prime} \mathrm{b}$ ), 3.28 (dt, 1H, J $=9.6,6.6 \mathrm{~Hz}$, octyl $\left.\mathrm{OCH}_{2}\right), 3.15\left(\mathrm{t}, 2 \mathrm{H}, J=7.0 \mathrm{~Hz}, \mathrm{CH}_{2} \mathrm{~N}_{3}\right), 1.54-$ $1.47\left(\mathrm{~m}, 4 \mathrm{H}\right.$, octyl $\left.\mathrm{CH}_{2}\right), 1.32-1.20\left(\mathrm{~m}, 8 \mathrm{H}\right.$, octyl $\left.\mathrm{CH}_{2}\right) .{ }^{13} \mathrm{C}$ NMR $\left(175 \mathrm{MHz}, \mathrm{CDCl}_{3}\right) \delta_{\mathrm{C}} 159.1(\mathrm{Ar}), 138.2(2 \mathrm{C}, \mathrm{Ar}), 138.1(\mathrm{Ar}), 138.0$ (Ar), 137.7 (2C, Ar), 136.8 (Ar), 135.9 (Ar), 131.4 (Ar), 131.3 (Ar), 130.2 (Ar), 129.3 (Ar), 129.1 (Ar), 128.4 (Ar), 128.3 (3C, Ar), 128.2 (Ar), 127.9 (2C, Ar), 127.8 (Ar), 127.7 (3C, Ar), $127.6(\mathrm{Ar}), 127.5$ (Ar), 127.4 (Ar), 113.7 (Ar), 106.8 (C-1"), $106.4\left(\mathrm{C}-1^{\prime}\right), 106.1$ (C1), $102.9\left(\mathrm{C}-1^{\prime \prime \prime}\right), 88.7$ (C-2), $88.3\left(\mathrm{C}-2^{\prime}\right), 86.4\left(\mathrm{C}-2^{\prime \prime}\right), 83.9$ (C-2'" $)$, 83.3, 83.2, 82.7, 82.0, 81.7, 81.2, 80.4, 80.2, 77.2, 77.1, 76.9, 73.3 $\left(\mathrm{OCH}_{2} \mathrm{Ph}\right), 72.6\left(\mathrm{OCH}_{2} \mathrm{Ph}\right), 72.3\left(\mathrm{OCH}_{2} \mathrm{Ph}\right), 72.2\left(\mathrm{OCH}_{2} \mathrm{Ph}\right), 72.0$, 71.8, $69.9\left(\mathrm{C}-5^{\prime \prime \prime}\right), 69.5,69.3,67.6$ (octyl $\left.\mathrm{OCH}_{2}\right), 66.0$ (2C, C-5, C$\left.5^{\prime}\right), 55.2\left(\mathrm{OCH}_{3}\right), 51.4\left(\mathrm{CH}_{2} \mathrm{~N}_{3}\right), 29.5\left(\right.$ octyl $\left.\mathrm{CH}_{2}\right), 29.3$ (octyl $\mathrm{CH}_{2}$ ), 29.1 (octyl $\mathrm{CH}_{2}$ ), 28.8 (octyl $\mathrm{CH}_{2}$ ), 26.7 (octyl $\mathrm{CH}_{2}$ ), 26.1 (octyl $\mathrm{CH}_{2}$ ). HRMS-ESI $\mathrm{m} / z[\mathrm{M}+\mathrm{Na}]^{+}$calcd for $\mathrm{C}_{85} \mathrm{H}_{97} \mathrm{~N}_{3} \mathrm{NaO}_{18}$ : 1470.6659. Found: 1470.6667 .

8-Azidooctyl 2,3-O-Xylylene- $\beta$-D-arabinofuranosyl- $(1 \rightarrow 2)-3,5$-di$O$-benzyl- $\alpha$-D-arabino-furanosyl-( $1 \rightarrow 5)$-2,3-di-O-benzyl- $\alpha$-D-arabinofuranosyl-( $1 \rightarrow 5)$-2,3-di-O-benzyl- $\alpha$-D-ara-binofuranoside (43). To a solution of $42(1.116 \mathrm{~g}, 0.76 \mathrm{mmol})$ in $\mathrm{CH}_{2} \mathrm{Cl}_{2}(50 \mathrm{~mL})$ was added TFA $(1.0 \mathrm{~mL})$. The reaction was monitored by TLC while the solution was stirred at rt. After $2 \mathrm{~h}$ the reaction was quenched by the addition of saturated aq. $\mathrm{NaHCO}_{3}$. The mixture was diluted with $\mathrm{CH}_{2} \mathrm{Cl}_{2}$ and washed with brine. The organic layer was subsequently dried with anhydrous $\mathrm{Na}_{2} \mathrm{SO}_{4}$, filtered, and concentrated, and the residue was purified by chromatography ( $3: 2$ hexanes-EtOAc) to give $43(0.93 \mathrm{~g}, 91 \%)$ as a syrup: $R_{\mathrm{f}} 0.58\left(3: 2\right.$, hexanes-EtOAc); $[\alpha]_{\mathrm{D}}$ $=+57.1\left(c=1.2, \mathrm{CHCl}_{3}\right) ;{ }^{1} \mathrm{H}$ NMR $\left(700 \mathrm{MHz}, \mathrm{CDCl}_{3}\right) \delta_{\mathrm{H}} 7.39-$ $7.21(\mathrm{~m}, 34 \mathrm{H}, \mathrm{Ar}), 5.15\left(\mathrm{~d}, 1 \mathrm{H}, J=1.0 \mathrm{~Hz}, \mathrm{H}-1^{\prime \prime}\right), 5.14\left(\mathrm{~s}, 1 \mathrm{H}, \mathrm{H}-1^{\prime}\right)$, $5.04\left(\mathrm{~d}, 1 \mathrm{H}, J=5.1 \mathrm{~Hz}, \mathrm{H}-1^{\prime \prime \prime}\right), 5.00(\mathrm{~s}, 1 \mathrm{H}, \mathrm{H}-1), 4.98(\mathrm{~d}, 1 \mathrm{H}, J=$ $\left.11.8 \mathrm{~Hz}, \mathrm{OCH}_{2} \mathrm{Ph}\right), 4.76-4.72\left(\mathrm{~m}, 3 \mathrm{H}, \mathrm{OCH}_{2} \mathrm{Ph}\right), 4.62(\mathrm{~d}, 1 \mathrm{H}, J=$ $\left.11.8 \mathrm{~Hz}, \mathrm{OCH}_{2} \mathrm{Ph}\right), 4.57-4.47\left(\mathrm{~m}, 8 \mathrm{H}, \mathrm{OCH}_{2} \mathrm{Ph}\right), 4.46(\mathrm{~d}, 1 \mathrm{H}, J=$ $\left.11.8 \mathrm{~Hz}, \mathrm{OCH}_{2} \mathrm{Ph}\right), 4.42\left(\mathrm{~d}, 1 \mathrm{H}, J=11.8 \mathrm{~Hz}, \mathrm{OCH}_{2} \mathrm{Ph}\right), 4.35-4.32$ (app t, $\left.J=6.3,1 \mathrm{H}, \mathrm{H}-3^{\prime \prime \prime}\right), 4.30$ (dd, $1 \mathrm{H}, J=3.6,1.4 \mathrm{~Hz}, \mathrm{H}-2^{\prime \prime}$ ), 4.18$4.14\left(\mathrm{~m}, 3 \mathrm{H}, \mathrm{H}-4^{\prime \prime}, \mathrm{H}-4^{\prime}, \mathrm{H}-4\right)$, 4.07-4.01 (m, 5H, H-2', H-3", H-3, H-3', H-2), 3.98-3.94 (m, 2H, H-4"', H-2"'), 3.89-3.85 (m, 2H, H5a, H-5'a), 3.72-3.62 (m, 4H, H-5b, H-5'b, octyl $\left.\mathrm{OCH}_{2}, \mathrm{H}-5^{\prime \prime \prime} \mathrm{a}\right)$, 3.60-3.58 (m, 2H, H-5"a, H-5"'b), 3.53 (dd, $1 \mathrm{H}, J=10.9,5.0 \mathrm{~Hz}, \mathrm{H}-$ $\left.5^{\prime \prime} \mathrm{b}\right), 3.37\left(\mathrm{dt}, 1 \mathrm{H}, J=9.6,6.6 \mathrm{~Hz}\right.$, octyl $\left.\mathrm{OCH}_{2}\right), 3.23(\mathrm{t}, 2 \mathrm{H}, J=7.0$ $\left.\mathrm{Hz}, \mathrm{CH}_{2} \mathrm{~N}_{3}\right), 2.59(\mathrm{~s}, 1 \mathrm{H}, \mathrm{OH}), 1.58\left(\mathrm{~m}, 4 \mathrm{H}\right.$, octyl $\left.\mathrm{CH}_{2}\right), 1.38-1.29$ $\left(\mathrm{m}, 8 \mathrm{H}\right.$, octyl $\left.\mathrm{CH}_{2}\right) .{ }^{13} \mathrm{C} \mathrm{NMR}\left(175 \mathrm{MHz}, \mathrm{CDCl}_{3}\right) \delta_{\mathrm{C}} 138.0(\mathrm{Ar})$, 138.0 (Ar), 137.9 (Ar), 137.6 (Ar), $137.0(\mathrm{Ar}), 135.5$ (Ar), 131.5 (Ar), 131.2 (Ar), 129.4 (2C, Ar), 128.4 (Ar), 128.3 (3C, Ar), 127.9 (Ar), 127.8 (2C, Ar), 127.7 (4C, Ar), 127.6 (3C, Ar), 106.6 (C-1" ), $106.4\left(\mathrm{C}-1^{\prime}\right), 106.1$ (C-1), 102.6 (C-1"' $), 88.7(\mathrm{C}-2), 88.2$ (C-2'), 86.8 (C-2"), 84.4 (C-2"'), 83.3 (3C, C-3, C-3', C-3"), 83.2 (C-4"'), 80.8, 80.4 (2C), 80.1, $73.4\left(\mathrm{OCH}_{2} \mathrm{Ph}\right), 72.3\left(\mathrm{OCH}_{2} \mathrm{Ph}\right), 72.2$ $\left(\mathrm{OCH}_{2} \mathrm{Ph}\right), 72.0\left(\mathrm{OCH}_{2} \mathrm{Ph}\right), 71.9\left(\mathrm{OCH}_{2} \mathrm{Ph}\right), 69.7\left(\mathrm{OCH}_{2} \mathrm{Ph}\right), 69.4$ $\left(\mathrm{C}-5^{\prime \prime}\right), 68.9\left(\mathrm{OCH}_{2} \mathrm{Ph}\right), 67.6\left(\right.$ octyl $\left.\mathrm{OCH}_{2}\right), 66.2\left(\mathrm{C}-5^{\prime}\right), 66.0(\mathrm{C}-5)$, $63.1\left(\mathrm{C}-5^{\prime \prime \prime}\right), 51.4\left(\mathrm{CH}_{2} \mathrm{~N}_{3}\right), 29.5$ (octyl $\left.\mathrm{CH}_{2}\right), 29.3$ (octyl $\left.\mathrm{CH}_{2}\right), 29.1$ (octyl $\left.\mathrm{CH}_{2}\right), 28.8$ (octyl $\left.\mathrm{CH}_{2}\right), 26.7$ (octyl $\left.\mathrm{CH}_{2}\right), 26.0$ (octyl $\mathrm{CH}_{2}$ ). 
HRMS-ESI $m / z[\mathrm{M}+\mathrm{Na}]^{+}$calcd for $\mathrm{C}_{78} \mathrm{H}_{91} \mathrm{~N}_{3} \mathrm{NaO}_{17}: 1364.6241$. Found: 1364.6238 .

8-Azidooctyl 6-O-Benzyl-2,3-Di-O-benzyol-4-O-levulinyl- $\alpha$-Dmannopyranosyl-( $1 \rightarrow 5)$-2,3-O-xylylene- $\beta$ - D-arabinofuranosyl- $(1 \rightarrow$ 2)-3,5-di-O-benzyl- $\alpha$-D-arabinofuranosyl- $(1 \rightarrow 5)-2,3$-di-O-benzyl- $\alpha$ $D$-arabinofuranosyl-(1 $\rightarrow 5)$-2,3-di-O-benzyl- $\alpha$-D-arabinofuranoside (44). To a mixture of donor $38(0.15 \mathrm{~g}, 0.22 \mathrm{mmol})$ and acceptor 43 $(0.2 \mathrm{~g}, 0.15 \mathrm{mmol})$ in $\mathrm{CH}_{2} \mathrm{Cl}_{2}(20 \mathrm{~mL})$ were added $4 \AA$ molecular sieves $(0.05 \mathrm{~g})$ at rt. After stirring for $1 \mathrm{~h}$ and then cooling to $-20{ }^{\circ} \mathrm{C}$, NIS (0.05 g, $0.23 \mathrm{mmol})$ and TMSOTf $(1.3 \mu \mathrm{L}, 0.0075 \mathrm{mmol})$ were added to the mixture, and the reaction was monitored by TLC. After stirring for $1 \mathrm{~h}$ at $-10^{\circ} \mathrm{C}$, the reaction was quenched by the addition of $\mathrm{Et}_{3} \mathrm{~N}$. The solution was diluted with $\mathrm{CH}_{2} \mathrm{Cl}_{2}$ and filtered through Celite. The filtrate was then washed with saturated aq. $\mathrm{Na}_{2} \mathrm{~S}_{2} \mathrm{O}_{3}$ and brine. The organic layer was subsequently dried with anhydrous $\mathrm{Na}_{2} \mathrm{SO}_{4}$, filtered, and concentrated, and the residue was purified by chromatography $(5: 1$, hexane-EtOAc) to give $44(0.23 \mathrm{~g}, 82 \%)$ as a white foam: $R_{\mathrm{f}} 0.3\left(3: 1\right.$, hexanes-EtOAc); $[\alpha]_{\mathrm{D}}=-0.3(c=0.2$, $\left.\mathrm{CHCl}_{3}\right) ;{ }^{1} \mathrm{H}$ NMR $\left(700 \mathrm{MHz}, \mathrm{CDCl}_{3}\right) \delta_{\mathrm{H}} 8.00(\mathrm{dd}, 2 \mathrm{H}, J=8.2,1.1$ $\mathrm{Hz}, \mathrm{Ar}$ ), 7.88 (dd, $2 \mathrm{H}, J=8.2,1.1 \mathrm{~Hz}, \mathrm{Ar}), 7.56-7.53(\mathrm{~m}, 1 \mathrm{H}, \mathrm{Ar})$, 7.50-7.46 (m, 1H, Ar), 7.38-7.15 (m, 42H, Ar), $7.11(\mathrm{t}, 1 \mathrm{H}, J=7.3$ $\mathrm{Hz}, \mathrm{Ar}), 5.82\left(\mathrm{t}, 1 \mathrm{H}, J=10.1 \mathrm{~Hz}, \mathrm{H}-4^{\prime \prime \prime \prime}\right), 5.66(\mathrm{dd}, 1 \mathrm{H}, J=10.1,3.3$ $\left.\mathrm{Hz}, \mathrm{H}-3^{\prime \prime \prime \prime}\right), 5.56$ (dd, $\left.1 \mathrm{H}, J=3.2,1.9 \mathrm{~Hz}, \mathrm{H}-2^{\prime \prime \prime \prime}\right), 5.12\left(\mathrm{~s}, 1 \mathrm{H}, \mathrm{H}-1^{\prime \prime}\right)$, $5.11\left(\mathrm{~s}, 1 \mathrm{H}, \mathrm{H}^{\prime} \mathrm{1}^{\prime}\right), 5.01$ (d, $\left.1 \mathrm{H}, J=4.8 \mathrm{~Hz}, \mathrm{H}-1^{\prime \prime \prime}\right), 4.99(\mathrm{~s}, 1 \mathrm{H}, \mathrm{H}-1)$, $4.94\left(\mathrm{~d}, 1 \mathrm{H}, J=12.6 \mathrm{~Hz}, \mathrm{OCH}_{2} \mathrm{Ph}\right), 4.87\left(\mathrm{~d}, 1 \mathrm{H}, J=1.2 \mathrm{~Hz}, \mathrm{H}-1^{\prime \prime \prime}\right)$, $4.85\left(\mathrm{~d}, 1 \mathrm{H}, J=12.6 \mathrm{~Hz}, \mathrm{OCH}_{2} \mathrm{Ph}\right), 4.83(\mathrm{~d}, 1 \mathrm{H}, J=12.6 \mathrm{~Hz}$, $\left.\mathrm{OCH}_{2} \mathrm{Ph}\right), 4.75\left(\mathrm{~d}, 1 \mathrm{H}, J=12.6 \mathrm{~Hz}, \mathrm{OCH}_{2} \mathrm{Ph}\right), 4.61-4.37(\mathrm{~m}, 15 \mathrm{H}$, $\left.\mathrm{OCH}_{2} \mathrm{Ph}\right), 4.25\left(\mathrm{dd}, 1 \mathrm{H}, J=3.3,1.2 \mathrm{~Hz}, \mathrm{H}-2^{\prime \prime}\right), 4.17-4.12(\mathrm{~m}, 5 \mathrm{H}$,

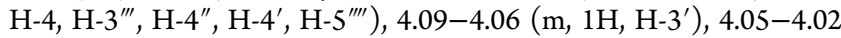
(m, 4H, H-2', H-3" $\left., \mathrm{H}-3, \mathrm{H}-3^{\prime}\right), 4.00$ (dd, $\left.1 \mathrm{H}, J=3.6,1.4 \mathrm{~Hz}, \mathrm{H}-2\right)$, 3.95-3.94 (m, 2H, H-2'", H-4'"), 3.87-3.84 (m, 2H, H-5a, H-5'a), 3.77 (dd, $\left.1 \mathrm{H}, J=10.3,6.0 \mathrm{~Hz}, \mathrm{H}-5^{\prime \prime \prime} \mathrm{a}\right), 3.70-3.65$ (m, 5H, H-5b, H$5^{\prime}$ b, H-6'"', octyl $\mathrm{OCH}_{2}$ ), 3.63 (dd, $\left.1 \mathrm{H}, J=10.3,8.2 \mathrm{~Hz}, \mathrm{H}-5^{\prime \prime \prime} \mathrm{b}\right), 3.58$ (dd, $\left.1 \mathrm{H}, J=10.8,3.7 \mathrm{~Hz}, \mathrm{H}-5^{\prime \prime} \mathrm{a}\right), 3.55$ (dd, $1 \mathrm{H}, J=10.8,5.5 \mathrm{~Hz}, \mathrm{H}-$ $\left.5^{\prime \prime} \mathrm{b}\right), 3.35\left(\mathrm{dt}, 1 \mathrm{H}, J=9.6,6.6 \mathrm{~Hz}\right.$, octyl $\left.\mathrm{OCH}_{2}\right), 3.22(\mathrm{t}, 2 \mathrm{H}, J=7.0$ $\left.\mathrm{Hz}, \mathrm{CH}_{2} \mathrm{~N}_{3}\right), 2.59-2.30\left(\mathrm{~m}, 4 \mathrm{H}, \mathrm{OC}(\mathrm{O}) \mathrm{CH}_{2} \mathrm{CH}_{2} \mathrm{C}(\mathrm{O})\right), 1.99(\mathrm{~s}, 3 \mathrm{H}$, $\left.\mathrm{C}(\mathrm{O}) \mathrm{CH}_{3}\right), 1.59-1.54\left(\mathrm{~m}, 4 \mathrm{H}\right.$, octyl $\left.\mathrm{CH}_{2}\right), 1.31\left(\mathrm{~m}, 8 \mathrm{H}\right.$, octyl $\left.\mathrm{CH}_{2}\right)$; ${ }^{13} \mathrm{C}$ NMR $\left(175 \mathrm{MHz}, \mathrm{CDCl}_{3}\right) \delta_{\mathrm{C}} 205.8(\mathrm{C}=\mathrm{O}), 171.6(\mathrm{C}=\mathrm{O})$, $165.3(\mathrm{C}=\mathrm{O}), 138.3(\mathrm{Ar}), 138.2(\mathrm{Ar}), 138.1(\mathrm{Ar}), 138.0(2 \mathrm{C}, \mathrm{Ar})$, 137.7 (2C, Ar), 136.9 (Ar), 135.8 (Ar), 133.3 (Ar), 133.1 (Ar), 131.6 (Ar), 131.2 (Ar), 129.9 (Ar), 129.8 (Ar), 129.4 (3C, Ar), 129.3 (Ar), 128.5 (Ar), 128.4 (Ar), 128.3 (4C, Ar), 128.2 (Ar), 127.9 (2C, Ar), 127.8 (Ar), 127.7 (3C, Ar), 127.6 (3C, Ar), 127.5 (2C, Ar), 127.4 (Ar), $106.6\left(\mathrm{C}-1^{\prime \prime}\right), 106.4\left(\mathrm{C}-1^{\prime}\right), 106.1(\mathrm{C}-1), 102.9$ (C-1"' $), 97.9$

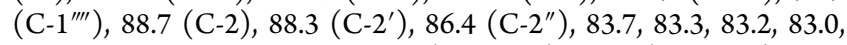
82.3, 81.1, 80.9, 80.5, 80.1, $73.6\left(\mathrm{OCH}_{2} \mathrm{Ph}\right), 73.3\left(\mathrm{OCH}_{2} \mathrm{Ph}\right), 72.3$ $\left(\mathrm{OCH}_{2} \mathrm{Ph}\right), 72.2\left(\mathrm{OCH}_{2} \mathrm{Ph}\right), 72.0\left(\mathrm{OCH}_{2} \mathrm{Ph}\right), 71.8\left(\mathrm{OCH}_{2} \mathrm{Ph}\right), 70.8$

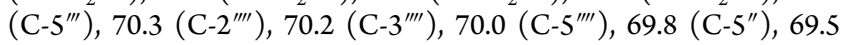
$\left(\mathrm{OCH}_{2} \mathrm{Ph}\right), 69.1\left(\mathrm{OCH}_{2} \mathrm{Ph}\right), 68.6\left(\mathrm{OCH}_{2} \mathrm{Ph}\right), 67.6$ (octyl $\left.\mathrm{OCH}_{2}\right)$, $66.7\left(\mathrm{C}-4^{\prime \prime \prime \prime}\right), 66.0\left(2 \mathrm{C}, \mathrm{C}-5, \mathrm{C}-5^{\prime}\right), 51.4\left(\mathrm{CH}_{2} \mathrm{~N}_{3}\right), 37.8(\mathrm{OC}(\mathrm{O})-$ $\mathrm{CH}_{2} \mathrm{CH}_{2} \mathrm{C}(\mathrm{O})$ ), 29.5 (octyl $\left.\mathrm{CH}_{2}\right), 29.5\left(\mathrm{C}(\mathrm{O}) \mathrm{CH}_{3}\right), 29.2$ (octyl $\left.\mathrm{CH}_{2}\right), 29.1$ (octyl $\left.\mathrm{CH}_{2}\right), 28.8$ (octyl $\left.\mathrm{CH}_{2}\right), 27.9\left(\left(\mathrm{OC}(\mathrm{O}) \mathrm{CH}_{2} \mathrm{CH}_{2} \mathrm{C}\right.\right.$ (O)), 26.6 (octyl $\mathrm{CH}_{2}$ ), 26.0 (octyl $\mathrm{CH}_{2}$ ). HRMS-ESI $\mathrm{m} / z[\mathrm{M}+$ $\mathrm{Na}]^{+}$calcd for $\mathrm{C}_{110} \mathrm{H}_{121} \mathrm{~N}_{3} \mathrm{NaO}_{26}: 1922.8131$. Found: 1922.8158 .

8-Azidooctyl-6-O-benzyl-2,3-di-O-benzoyl- $\alpha$-D-mannopyranosyl-(1 $\rightarrow 5)-2,3-O$-xylylene- $\beta$-D-arabinofuranosyl- $(1 \rightarrow 2)-3,5-d i-O-$ benzyl- $\alpha$-D-arabinofuranosyl- $(1 \rightarrow 5)-2,3-d i-O$-benzyl- $\alpha$ - $D$-arabinofuranosyl-(1 $\rightarrow 5)-2,3-d i-O$-benzyl- $\alpha$-D-arabinofuranoside (45). A solution of $44(78 \mathrm{mg}, 0.041 \mathrm{mmol})$ and hydrazine acetate $(7.5$ $\mathrm{mg}, 0.082 \mathrm{mmol})$ in $1: 1 \mathrm{CH}_{2} \mathrm{Cl}_{2}-\mathrm{CH}_{3} \mathrm{OH}(6 \mathrm{~mL})$ was stirred at $\mathrm{rt}$ for $4 \mathrm{~h}$. Then the solution was concentrated, and the resulting residue was purified by chromatography $\left(2: 1: 1\right.$, hexanes-EtOAc- $\left.\mathrm{CH}_{2} \mathrm{Cl}_{2}\right)$ to afford $45(0.07 \mathrm{~g}, 95 \%)$ as a white foam: $R_{\mathrm{f}} 0.2$ (3:1, hexanesEtOAc); $[\alpha]_{\mathrm{D}}=+21.4\left(c=0.2, \mathrm{CHCl}_{3}\right) ;{ }^{1} \mathrm{H}$ NMR $(700 \mathrm{MHz}$, $\left.\mathrm{CDCl}_{3}\right) \delta_{\mathrm{H}} 8.05(\mathrm{~d}, 2 \mathrm{H}, J=7.4 \mathrm{~Hz}), 7.92(\mathrm{~d}, 2 \mathrm{H}, J=7.4 \mathrm{~Hz}), 7.59(\mathrm{t}$, $1 \mathrm{H}, J=7.4 \mathrm{~Hz}), 7.50(\mathrm{t}, 1 \mathrm{H}, J=7.4 \mathrm{~Hz}), 7.43-7.18(\mathrm{~m}, 42 \mathrm{H}), 7.13$ $(\mathrm{t}, 1 \mathrm{H}, J=7.3 \mathrm{~Hz}), 5.56-4.54\left(\mathrm{~m}, 2 \mathrm{H}, \mathrm{H}-3^{\prime \prime \prime \prime}, \mathrm{H}-2^{\prime \prime \prime \prime}\right), 5.14(\mathrm{~s}, 1 \mathrm{H}, \mathrm{H}-$ $\left.1^{\prime \prime}\right), 5.13\left(\mathrm{~s}, 1 \mathrm{H}, \mathrm{H}-1^{\prime}\right), 5.04\left(\mathrm{~d}, 1 \mathrm{H}, J=4.9 \mathrm{~Hz}, \mathrm{H}-1^{\prime \prime \prime}\right), 5.00(\mathrm{~s}, 1 \mathrm{H}$, $\mathrm{H}-1), 4.96\left(\mathrm{~d}, 1 \mathrm{H}, J=12.6 \mathrm{~Hz}, \mathrm{OCH}_{2} \mathrm{Ph}\right), 4.86\left(\mathrm{~s}, 1 \mathrm{H}, \mathrm{H}-1^{\prime \prime \prime \prime}\right), 4.84$ $\left(\mathrm{d}, 1 \mathrm{H}, J=12.6 \mathrm{~Hz}, \mathrm{OCH}_{2} \mathrm{Ph}\right), 4.81\left(\mathrm{~d}, 1 \mathrm{H}, J=12.6 \mathrm{~Hz}, \mathrm{OCH}_{2} \mathrm{Ph}\right)$, $4.76\left(\mathrm{~d}, 1 \mathrm{H}, J=12.6 \mathrm{~Hz}, \mathrm{OCH}_{2} \mathrm{Ph}\right), 4.66(\mathrm{~d}, 1 \mathrm{H}, J=11.8 \mathrm{~Hz}$, $\mathrm{OCH}_{2} \mathrm{Ph}$ ), $4.62\left(\mathrm{~d}, 1 \mathrm{H}, J=11.8 \mathrm{~Hz}, \mathrm{OCH}_{2} \mathrm{Ph}\right), 4.58-4.45(\mathrm{~m}, 10 \mathrm{H}$, $\left.\mathrm{OCH}_{2} \mathrm{Ph}\right), 4.41\left(\mathrm{~d}, 1 \mathrm{H}, J=11.8 \mathrm{~Hz}, \mathrm{OCH}_{2} \mathrm{Ph}\right), 4.38(\mathrm{t}, 1 \mathrm{H}, J=9.5$ $\left.\mathrm{Hz}, \mathrm{H}-4^{\prime \prime \prime \prime}\right), 4.28$ (d, 1H, J = 2.5 Hz, H-2" ), 4.18-4.14 (m, 4H, H-3"', H-4, H-4", H-4'), 4.11-4.07 (m, 1H, H-3'), 4.07-4.00 (m, 5H, H-2', H-3", H-5'"', H-3, H-2), 3.99-3.95 (m, 2H, H-2'"', H-4"'), 3.92 (dd, $\left.1 \mathrm{H}, J=10.5,3.8 \mathrm{~Hz}, \mathrm{H}-6^{\prime \prime \prime \prime} \mathrm{a}\right), 3.88-3.85$ (m, 2H, H-5a, H-5'a), 3.783.74 (m, 2H, H-6"''b, H-5'"'a), 3.71-3.67 (m, 3H, H-5'a, H-5'b, octyl $\mathrm{OCH}_{2}$ ), $3.64\left(\mathrm{dd}, 1 \mathrm{H}, J=10.3,7.8 \mathrm{~Hz}, \mathrm{H}-5^{\prime \prime \prime} \mathrm{b}\right), 3.60$ (dd, $1 \mathrm{H}, J=$ 10.8, 3.7 Hz, H-5"a), 3.57 (dd, $1 \mathrm{H}, J=10.8,5.4 \mathrm{~Hz}, \mathrm{H}-5{ }^{\prime \prime} \mathrm{b}$ ), 3.36 (dt, $1 \mathrm{H}, J=9.5,6.7 \mathrm{~Hz}$, octyl $\left.\mathrm{OCH}_{2}\right), 3.23\left(\mathrm{t}, 2 \mathrm{H}, J=7.0 \mathrm{~Hz}, \mathrm{CH}_{2} \mathrm{~N}_{3}\right)$, $2.90-2.84(\mathrm{~m}, 1 \mathrm{H}, \mathrm{OH}), 1.60-1.55\left(\mathrm{~m}, 4 \mathrm{H}\right.$, octyl $\left.\mathrm{CH}_{2}\right), 1.36-1.30$

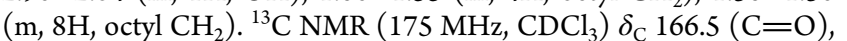
$165.4(\mathrm{C}=\mathrm{O}), 138.2$ (Ar), 138.1 (2C, Ar), 138.0 (2C, Ar), 137.7 (2C)), $136.9(\mathrm{Ar}), 135.8(\mathrm{Ar}), 133.3(\mathrm{Ar}), 133.2(\mathrm{Ar}), 131.6(\mathrm{Ar})$, 131.3 (Ar), $129.9(\mathrm{Ar}), 129.8(\mathrm{Ar}), 129.6(\mathrm{Ar}), 129.5(\mathrm{Ar}), 129.3$ (Ar), 128.5 (Ar), 128.4 (Ar), 128.3 (4C, Ar), 127.9 (2C, Ar), 127.8 (Ar), 127.7 (2C, Ar), 127.6 (2C, Ar), 127.5 (2C, Ar), 106.5 (C-1" ), $106.4\left(\mathrm{C}-1^{\prime}\right), 106.1(\mathrm{C}-1), 102.8\left(\mathrm{C}-1^{\prime \prime \prime \prime}\right), 98.1(\mathrm{C}-2), 88.7\left(\mathrm{C}-2^{\prime}\right)$, 88.3 (C-2"), 86.3, 83.7, 83.3, 83.2, 82.8, 82.3, 81.1, 81.0, 80.4, 80.1, $73.8\left(\mathrm{OCH}_{2} \mathrm{Ph}\right), 73.3\left(\mathrm{OCH}_{2} \mathrm{Ph}\right), 72.8\left(\mathrm{C}-3^{\prime \prime \prime \prime}\right), 72.3\left(\mathrm{OCH}_{2} \mathrm{Ph}\right), 72.2$ $\left(\mathrm{OCH}_{2} \mathrm{Ph}\right), 72.0\left(\mathrm{OCH}_{2} \mathrm{Ph}\right), 71.8\left(\mathrm{OCH}_{2} \mathrm{Ph}\right), 71.5\left(\mathrm{OCH}_{2} \mathrm{Ph}\right), 70.7$

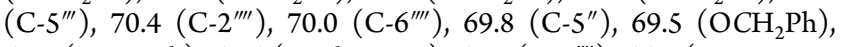
$69.2\left(\mathrm{OCH}_{2} \mathrm{Ph}\right), 67.6\left(\right.$ octyl $\left.\mathrm{OCH}_{2}\right), 67.4\left(\mathrm{C}-4^{\prime \prime \prime \prime}\right), 66.0(2 \mathrm{C}, \mathrm{C}-5, \mathrm{C}-$ 5'), $51.4\left(\mathrm{CH}_{2} \mathrm{~N}_{3}\right), 29.5$ (octyl $\left.\mathrm{CH}_{2}\right), 29.3\left(\right.$ octyl $\left.\mathrm{CH}_{2}\right), 29.1$ (octyl $\left.\mathrm{CH}_{2}\right), 28.8\left(\right.$ octyl $\left.\mathrm{CH}_{2}\right), 26.7$ (octyl $\left.\mathrm{CH}_{2}\right), 26.0\left(\right.$ octyl $\left.\mathrm{CH}_{2}\right)$. HRMSESI $m / z\left[\mathrm{M}+\mathrm{NH}_{4}\right]^{+}$calcd for $\mathrm{C}_{105} \mathrm{H}_{115} \mathrm{~N}_{3} \mathrm{NaO}_{24}: 1824.7763$. Found: 1824.7784 .

8-Azidooctyl 5-O-Methanesulfonyl-2,3-O-xylylene- $\alpha$-D-xylofuranosyl-(1 $\rightarrow 4)-6$-O-benzyl-2,3-di-O-benzyol- $\alpha$-D-mannopyranosyl$(1 \rightarrow 5)-2,3-O-x y l y l e n e-\beta$-D-arabinofuranosyl- $(1 \rightarrow 2)-3,5$-di-O-benzyl- $\alpha$-D-arabinofuranosyl- $(1 \rightarrow 5)-2,3$-di-O-benzyl- $\alpha$-D-arabinofuranosyl-( $1 \rightarrow 5)-2,3-d i-O-b e n z y l-\alpha$-D-arabinofuranoside (46). To a mixture of donor $37(26 \mathrm{mg}, 0.06 \mathrm{mmol})$ and acceptor $45(54 \mathrm{mg}$, $0.03 \mathrm{mmol})$ in THF $(6 \mathrm{~mL})$ were added $4 \AA$ molecular sieves $(60 \mathrm{mg})$ at rt. After stirring for $0.5 \mathrm{~h}$ and then cooling to $0{ }^{\circ} \mathrm{C}$, NIS $(25 \mathrm{mg}$, $0.075 \mathrm{mmol})$ and TMSOTf $(1 \mu \mathrm{L}, 0.004 \mathrm{mmol})$ were added. After stirring overnight at $\mathrm{rt}$, the reaction was quenched by the addition of $\mathrm{Et}_{3} \mathrm{~N}$. The solution was diluted with $\mathrm{CH}_{2} \mathrm{Cl}_{2}$ and filtered through Celite. The filtrate was then washed with saturated aq. $\mathrm{Na}_{2} \mathrm{~S}_{2} \mathrm{O}_{3}$ and brine. The organic layer was subsequently dried with anhydrous $\mathrm{Na}_{2} \mathrm{SO}_{4}$, filtered, and concentrated, and the residue was purified by chromatography $(2: 1$, hexane-EtOAc) to give $46(55 \mathrm{mg}, 87 \%)$ as a white foam: $R_{\mathrm{f}} 0.31\left(3: 2\right.$, hexanes-EtOAc); $[\alpha]_{\mathrm{D}}=+10.7(c=0.4$, $\left.\mathrm{CHCl}_{3}\right) ;{ }^{1} \mathrm{H}$ NMR $\left(500 \mathrm{MHz}, \mathrm{CDCl}_{3}\right) \delta_{\mathrm{H}} 7.94-7.84(\mathrm{~m}, 4 \mathrm{H}, \mathrm{Ar})$, 7.54-7.43 (m, 2H, Ar), 7.37-7.08 (m, 50H, Ar), 7.03 (ddd, $2 \mathrm{H}, J=$ 12.2, 6.0, $2.3 \mathrm{~Hz}, \mathrm{Ar}), 6.52-6.42(\mathrm{~m}, 1 \mathrm{H}, \mathrm{Ar}), 5.72(\mathrm{dd}, 1 \mathrm{H}, J=9.8$, $\left.3.2 \mathrm{~Hz}, \mathrm{H}-3^{\prime \prime \prime \prime}\right), 5.45$ (dd, $\left.1 \mathrm{H}, J=3.2,1.9 \mathrm{~Hz}, \mathrm{H}-2^{\prime \prime \prime \prime}\right), 5.14(\mathrm{~d}, 1 \mathrm{H}, J=$ $5.0 \mathrm{~Hz}, \mathrm{H}-1^{\prime \prime \prime \prime \prime}$ ) , 5.05-5.04 (m, 2H, H-1", $\left.\mathrm{H}-1^{\prime}\right), 4.95$ (d, 1H, J = 5.0 $\left.\mathrm{Hz}, \mathrm{H}-1^{\prime \prime \prime}\right), 4.92(\mathrm{~d}, 1 \mathrm{H}, J=1.0 \mathrm{~Hz}, \mathrm{H}-1), 4.87(\mathrm{~d}, 1 \mathrm{H}, J=12.6 \mathrm{~Hz}$, $\mathrm{OCH}_{2} \mathrm{Ph}$ ), 4.79 (d, $\left.1 \mathrm{H}, J=12.6 \mathrm{~Hz}, \mathrm{OCH}_{2} \mathrm{Ph}\right), 4.76(\mathrm{~d}, 1 \mathrm{H}, J=12.6$ $\mathrm{Hz}, \mathrm{OCH}_{2} \mathrm{Ph}$ ), 4.75 (d, $\left.1 \mathrm{H}, J=1.7 \mathrm{~Hz}, \mathrm{H}-1^{\prime \prime \prime \prime}\right), 4.70$ (d, $1 \mathrm{H}, J=12.7$ $\left.\mathrm{Hz}, \mathrm{OCH}_{2} \mathrm{Ph}\right), 4.58-4.30$ (m, $\left.13 \mathrm{H}, \mathrm{OCH}_{2} \mathrm{Ph}, \mathrm{H}-4^{\prime \prime \prime \prime}, \mathrm{H}-4^{\prime \prime \prime \prime \prime}\right), 4.25$ (dd, $\left.1 \mathrm{H}, J=11.4,2.8 \mathrm{~Hz}, \mathrm{H}-5^{\prime \prime \prime \prime \prime} \mathrm{a}\right), 4.18$ (dd, $1 \mathrm{H}, J=3.2,1.2 \mathrm{~Hz}, \mathrm{H}-$ $2^{\prime \prime}$ ), 4.13 (dd, $\left.1 \mathrm{H}, J=7.7,5.4 \mathrm{~Hz}, \mathrm{H}-3^{\prime \prime \prime \prime \prime}\right), 4.16-3.96$ (m, 9H, H-3"', H-4, H-5", H-4', H-5'"'' 'b, H-3", H-5 ${ }^{\prime \prime \prime \prime}, \mathrm{H}-3, \mathrm{H}-2^{\prime}$ ), 3.94 (dd, 1H, J = 3.6, $1.4 \mathrm{~Hz}, \mathrm{H}-2)$, 3.91-3.85 (m, 2H, H-4"', H-2'"'), 3.81-3.75 (m, $\left.3 \mathrm{H}, \mathrm{H}-5 \mathrm{a}, \mathrm{H}-5^{\prime} \mathrm{a}, \mathrm{H}-6^{\prime \prime \prime \prime} \mathrm{a}\right), 3.70$ (dd, $\left.1 \mathrm{H}, J=10.1,5.9 \mathrm{~Hz}, \mathrm{H}-5^{\prime \prime \prime} \mathrm{a}\right)$,

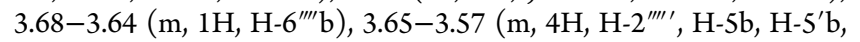
octyl $\left.\mathrm{OCH}_{2}\right), 3.55-3.46\left(\mathrm{~m}, 3 \mathrm{H}, \mathrm{H}-6^{\prime \prime \prime} \mathrm{b}, \mathrm{H}-5^{\prime \prime}\right), 3.29(\mathrm{dt}, 1 \mathrm{H}, J=9.7$, $6.7 \mathrm{~Hz}$, octyl $\left.\mathrm{OCH}^{2}\right), 3.16\left(\mathrm{t}, 2 \mathrm{H}, J=7.0 \mathrm{~Hz}, \mathrm{CH}_{2} \mathrm{~N}_{3}\right), 2.68(\mathrm{~s}, 3 \mathrm{H}$, $\left.\mathrm{S}(\mathrm{O})_{2} \mathrm{CH}_{3}\right), 1.53-1.43\left(\mathrm{~m}, 4 \mathrm{H}\right.$, octyl $\left.\mathrm{CH}_{2}\right), 1.31-1.21$ (m, $8 \mathrm{H}$, octyl $\left.\mathrm{CH}_{2}\right) ;{ }^{13} \mathrm{C}$ NMR $\left(125 \mathrm{MHz}, \mathrm{CDCl}_{3}\right) \delta_{\mathrm{C}} 165.2(\mathrm{C}=\mathrm{O}), 165.0(\mathrm{C}=$ $\mathrm{O}), 138.8(\mathrm{Ar}), 138.2(\mathrm{Ar}), 138.1(\mathrm{Ar}), 138.1(\mathrm{Ar}), 138.0(\mathrm{Ar}), 137.7$ (2C, Ar), 137.0 (Ar), 136.0 (Ar), 135.8 (Ar), 135.2 (Ar), 133.2 (Ar), 132.8 (Ar), 131.7 (Ar), 131.3 (2C, Ar), 130.3 (Ar), 129.8 (Ar), 129.6 (Ar), 129.5 (Ar), 129.4 (Ar), 129.3 (2C, Ar), 128.5 (2C, Ar), 128.4 (2C, Ar), 128.3 (4C, Ar), 127.9 (2C, Ar), 127.8 (2C, Ar), 127.7 (3C, $\mathrm{Ar}), 127.6(\mathrm{Ar}), 127.5$ (2C, Ar), 127.3 (Ar), 106.7 (C-1"), 106.4 (C-

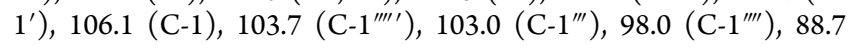


(C-2), 88.3 (C-2'), 86.4 (C-2" ) , 83.8, 83.4, 83.2, 82.9, 82.6, 81.8, 81.2, 80.9, 80.5, 80.2, 80.0, $76.1\left(\mathrm{C}-4^{\prime \prime \prime \prime \prime}\right), 73.7\left(\mathrm{C}-4^{\prime \prime \prime \prime}\right), 73.4$ $\left(\mathrm{OCH}_{2} \mathrm{Ph}\right), 73.3\left(\mathrm{OCH}_{2} \mathrm{Ph}\right), 72.3\left(\mathrm{OCH}_{2} \mathrm{Ph}\right), 72.2\left(\mathrm{OCH}_{2} \mathrm{Ph}\right), 72.0$ (2C, C-3'"', $\left.\mathrm{OCH}_{2} \mathrm{Ph}\right), 71.8,71.2,70.7$ (2C, C-5"', C-2'"') $), 69.9$ (C$\left.5^{\prime \prime}\right), 69.5\left(\mathrm{OCH}_{2} \mathrm{Ph}\right), 69.2\left(\mathrm{OCH}_{2} \mathrm{Ph}\right), 69.1\left(\mathrm{OCH}_{2} \mathrm{Ph}\right), 69.0$ $\left(\mathrm{OCH}_{2} \mathrm{Ph}\right), 68.7\left(\mathrm{OCH}_{2} \mathrm{Ph}\right), 67.6$ (octyl $\left.\mathrm{OCH}_{2}\right), 66.0(2 \mathrm{C}, \mathrm{C}-5$, C5'), $51.5\left(\mathrm{CH}_{2} \mathrm{~N}_{3}\right), 37.2\left(\mathrm{~S}(\mathrm{O})_{2} \mathrm{CH}_{3}\right), 29.5$ (octyl $\left.\mathrm{CH}_{2}\right), 29.3$ (octyl $\left.\mathrm{CH}_{2}\right), 29.1$ (octyl $\left.\mathrm{CH}_{2}\right), 28.8$ (octyl $\left.\mathrm{CH}_{2}\right), 26.7$ (octyl $\left.\mathrm{CH}_{2}\right), 26.1$ (octyl $\mathrm{CH}_{2}$ ). HRMS-ESI $m / z[\mathrm{M}+\mathrm{Na}]^{+}$calcd for $\mathrm{C}_{119} \mathrm{H}_{131} \mathrm{~N}_{3} \mathrm{NaO}_{30} \mathrm{~S}$ : 2136.8430. Found: 2136.8446.

8-Azidooctyl 5-Deoxy-5-methylthio-2,3-O-xylylene- $\alpha$-D-xylofuranosyl-( $1 \rightarrow 4)-6$-O-benzyl- $\alpha$-D-mannopyranosyl- $(1 \rightarrow 5)-2,3-0$-xylylene- $\beta$-D-arabinofuranosyl-(1 $\rightarrow 2)$-3,5-di-O-benzyl- $\alpha$-D-arabinofuranosyl- $(1 \rightarrow 5)-2,3-d i-O-$ benzyl- $\alpha$-D-arabinofuranosyl- $(1 \rightarrow 5)-2,3-d i-$ O-benzyl- $\alpha$-D-arabinofuranoside (47). To a solution of $46(40 \mathrm{mg}$, $0.0189 \mathrm{mmol})$ in $\mathrm{CH}_{3} \mathrm{CN}(2 \mathrm{~mL})$ was added sodium thiomethoxide ( $4 \mathrm{mg}, 0.057 \mathrm{mmol}$ ). The reaction mixture was heated at reflux for $3 \mathrm{~h}$ and then cooled to $\mathrm{rt}$ before being diluted with $\mathrm{CH}_{3} \mathrm{CN}(8 \mathrm{~mL})$ and filtered through Celite. The filtrate was concentrated to a syrup that was purified by chromatography $(1: 1$, hexanes-EtOAc) to afford 47 $(26 \mathrm{mg}, 74 \%)$ as an oil. $R_{\mathrm{f}} 0.2\left(1: 1\right.$, hexane-EtOAc); $[\alpha]_{\mathrm{D}}=+52.1(\mathrm{c}$ $\left.=0.2, \mathrm{CHCl}_{3}\right) ;{ }^{1} \mathrm{H} \mathrm{NMR}\left(700 \mathrm{MHz}, \mathrm{CDCl}_{3}\right) \delta_{\mathrm{H}} 7.33(\mathrm{qd}, 2 \mathrm{H}, J=7.5$, $3.8 \mathrm{~Hz}), 7.29-7.07(\mathrm{~m}, 41 \mathrm{H}), 5.05\left(\mathrm{~s}, 2 \mathrm{H}, \mathrm{H}-1^{\prime}, \mathrm{H}-1^{\prime \prime}\right), 5.02(\mathrm{~d}, 1 \mathrm{H}, J$ $\left.=4.8 \mathrm{~Hz}, \mathrm{H}-1^{\prime \prime \prime \prime \prime}\right), 4.92(\mathrm{~s}, 1 \mathrm{H}, \mathrm{H}-1), 4.91\left(\mathrm{~d}, 1 \mathrm{H}, J=5.0 \mathrm{~Hz}, \mathrm{H}-1^{\prime \prime \prime}\right)$, $4.88\left(\mathrm{~d}, 1 \mathrm{H}, J=12.6 \mathrm{~Hz}, \mathrm{OCH}_{2} \mathrm{Ph}\right), 4.85(\mathrm{~d}, 1 \mathrm{H}, J=12.6 \mathrm{~Hz}$, $\left.\mathrm{OCH}_{2} \mathrm{Ph}\right), 4.82-4.75\left(\mathrm{~m}, 3 \mathrm{H}, \mathrm{OCH}_{2} \mathrm{Ph}\right), 4.72(\mathrm{~d}, 1 \mathrm{H}, J=0.6 \mathrm{~Hz}, \mathrm{H}-$ $\left.1^{\prime \prime \prime \prime}\right), 4.70-4.62\left(\mathrm{~m}, 4 \mathrm{H}, \mathrm{OCH}_{2} \mathrm{Ph}\right), 4.53(\mathrm{~d}, 1 \mathrm{H}, J=11.9 \mathrm{~Hz}$, $\left.\mathrm{OCH}_{2} \mathrm{Ph}\right), 4.50-4.39\left(\mathrm{~m}, 10 \mathrm{H}, \mathrm{OCH}_{2} \mathrm{Ph}\right), 4.36-4.32(\mathrm{~m}, 3 \mathrm{H}, \mathrm{H}-$ $\left.4^{\prime \prime \prime \prime \prime}\right), 4.17\left(\mathrm{dd}, 1 \mathrm{H}, J=3.1,1.1 \mathrm{~Hz}, \mathrm{H}-2^{\prime \prime}\right), 4.07\left(\mathrm{~m}, 4 \mathrm{H}, \mathrm{H}-3^{\prime \prime \prime \prime \prime}\right)$,

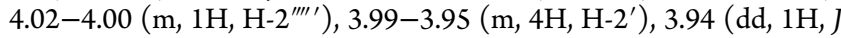

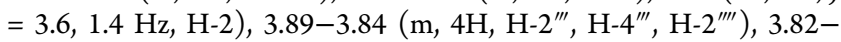
3.75 (m, 4H, H-5'"', H-4'"', H-5a, H-5'a), 3.73 (dd, 1H, J = 11.6, 4.7

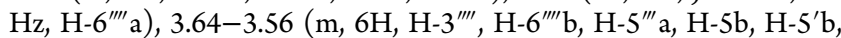
octyl $\left.\mathrm{OCH}_{2}\right), 3.52-3.48$ (m, 2H, H-5"a, H-5"'b), 3.46 (dd, $1 \mathrm{H}, J=$ $\left.10.8,5.5 \mathrm{~Hz}, \mathrm{H}-5^{\prime \prime} \mathrm{b}\right), 3.29\left(\mathrm{dt}, 1 \mathrm{H}, J=9.6,6.6 \mathrm{~Hz}\right.$, octyl $\left.\mathrm{OCH}_{2}\right), 3.16$ $\left(\mathrm{t}, 2 \mathrm{H}, J=7.0 \mathrm{~Hz}, \mathrm{CH}_{2} \mathrm{~N}_{3}\right), 2.68\left(\mathrm{dd}, 1 \mathrm{H}, J=14.0,4.9 \mathrm{~Hz}, \mathrm{H}-5^{\prime \prime \prime \prime \prime} \mathrm{a}\right)$, 2.52 (dd, $\left.1 \mathrm{H}, J=14.0,7.8 \mathrm{~Hz}, \mathrm{H}-5^{\prime \prime \prime \prime \prime} ' \mathrm{~b}\right), 2.03$ (s, 3H, $\mathrm{SCH}_{3}$ ), 1.54$1.47\left(\mathrm{~m}, 4 \mathrm{H}\right.$, octyl $\left.\mathrm{CH}_{2}\right), 1.28-1.21\left(\mathrm{~m}, 8 \mathrm{H}\right.$, octyl $\left.\mathrm{CH}_{2}\right) ;{ }^{13} \mathrm{C}$ NMR $\left(125 \mathrm{MHz}, \mathrm{CDCl}_{3}\right) \delta_{\mathrm{C}} 138.6(\mathrm{Ar}), 138.2(\mathrm{Ar}), 138.1$ (2C, $\left.\mathrm{Ar}\right), 138.0$ (Ar), 137.7 (2C, Ar), 136.8 (Ar), $136.4(\mathrm{Ar}), 135.8$ (Ar), 135.1 (Ar), 131.7 (Ar), 131.6 (Ar), 131.5 (Ar), 131.2 (Ar), 130.0 (Ar), 129.7 (Ar), 129.4 (Ar), 129.3 (Ar), $128.4(\mathrm{Ar}), 128.3$ (4C, Ar), $128.2(\mathrm{Ar})$, 127.9 ( $\mathrm{Ar}), 127.8(\mathrm{Ar}), 127.7$ (4C, Ar), 127.6 (Ar), 127.5 (2C, Ar), 127.3 (Ar), $106.8\left(\mathrm{C}-1^{\prime \prime}\right), 106.4\left(\mathrm{C}-1^{\prime}\right), 106.1(\mathrm{C}-1), 104.1\left(\mathrm{C}-1^{\prime \prime \prime \prime}\right)$, 103.0 (C-1"'), 99.7 (C-1'”'), 88.7 (C-2), 88.3 (C-2'), 86.9 (C-2"), 84.1, 83.8, 83.3, 83.2, 82.2, 82.1, 81.2, 81.0, 80.4, 80.2, 79.8, 79.1, 78.6, 73.4, 73.3, 72.3, 72.1, 72.0, 71.9, 70.9, 70.8, 70.3, 69.7, 69.6, 69.5, 69.2, 69.2, 68.9, 68.8, 67.6 (octyl $\left.\mathrm{OCH}_{2}\right), 66.0\left(\mathrm{C}-5^{\prime}\right), 65.9$ (C-

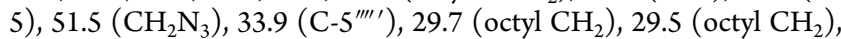
29.3 (octyl $\mathrm{CH}_{2}$ ), 29.1 (octyl $\mathrm{CH}_{2}$ ), 28.8 (octyl $\mathrm{CH}_{2}$ ), 26.7 (octyl $\left.\mathrm{CH}_{2}\right), 26.1\left(\right.$ octyl $\left.\mathrm{CH}_{2}\right), 16.8\left(\mathrm{SCH}_{3}\right)$. HRMS-ESI $m / z\left[\mathrm{M}+\mathrm{NH}_{4}\right]^{+}$ calcd for $\mathrm{C}_{105} \mathrm{H}_{127} \mathrm{~N}_{4} \mathrm{O}_{25} \mathrm{~S}$ : 1875.8505 . Found: 1875.8503 .

Computational Methodology. All calculations were performed in the Gaussian 09 program. ${ }^{28}$ Geometry optimizations were run for oxocarbenium ion 34, as well as the 1,2-cis product and 1,2-trans products of its trapping with methanol at the $\mathrm{HF} / 6-311 \mathrm{G}(\mathrm{d}, \mathrm{p})^{29-32} / /$ B3LYP/6-31G $(d)^{33,34}$ level of theory. Using the optimized geometries of the two products, a reaction coordinate scan was carried out on the C1-O 1 bond from 1.5 to $2.4 \AA$ to obtain a picture of the energetics of the reaction coordinate. Each of the scan structures was used as input for transition state calculations using the HF/6-31G(d) ${ }^{29,34}$ level of theory. Frequency analysis showed that one imaginary frequency was obtained for each of the structures, as described above. All calculations were done in the gas phase.

\section{ASSOCIATED CONTENT}

\section{S Supporting Information}

The Supporting Information is available free of charge on the ACS Publications website at DOI: 10.1021/acs.joc.8b00410.
${ }^{1} \mathrm{H}$ and ${ }^{13} \mathrm{C}$ NMR spectra for all new compounds; Cartesian coordinates for oxacarbenium ion 34 and glycosides that would result from its trapping with methanol (PDF)

Movie showing the approach of methanol to 34, leading to the 1,2-cis product

(MPG)

Movie showing the approach of methanol to 34, leading to the 1,2-trans product

(MPG)

\section{AUTHOR INFORMATION}

\section{Corresponding Author}

*E-mail: tlowary@ualberta.ca

ORCID $\odot$

Todd L. Lowary: 0000-0002-8331-8211

Notes

The authors declare no competing financial interest.

\section{ACKNOWLEDGMENTS}

This work was supported by the Bill and Melinda Gates Foundation, the Alberta Glycomics Centre, the Canadian Glycomics Network, and the Natural Sciences and Engineering Research Council of Canada. We thank Dr. Michele Richards and Dr. Maju Joe for assistance in the preparation of the manuscript.

\section{REFERENCES}

(1) Marino, C.; Gallo-Rodriguez, C.; de Lederkremer, R. M. Galactofuranosyl-Containing Glycans: Occurrence, Synthesis and Biochemistry. In Glycans: Biochemistry, Characterization and Applications, first ed.; Mora-Montes, H. M., Ed.; Nova Science Publishers, Inc.: Hauppauge, NY, 2012; pp 207-268.

(2) Richards, M. R.; Lowary, T. L. Chemistry and Biology of Galactofuranose-Containing Polysaccharides. ChemBioChem 2009, 10, 1920-1938.

(3) Angala, S. K.; Belardinelli, J. M.; Huc-Claustre, E.; Wheat, W. H.; Jackson, M. The Cell Envelope Glycoconjugates of Mycobacterium tuberculosis. Crit. Rev. Biochem. Mol. Biol. 2014, 49, 361-399.

(4) Tefsen, B.; van Die, I. Glycosyltransferases in Chemo-enzymatic Synthesis of Oligosaccharides. Methods Mol. Biol. 2013, 1022, 357367.

(5) Imamura, A.; Lowary, T. L. Chemical Synthesis of Furanose Glycosides. Trends Glycosci. Glycotechnol. 2011, 23, 134-152.

(6) Gallo-Rodriguez, C.; Kashiwagi, G. A. Selective Glycosylations with Furanosides. In Selective Glycosylations: Synthetic Methods and Catalysts; 1st ed.; Bennett, C. S., Ed.; Wiley-VCH Verlag GmbH \& Co, KGaA: New York, 2017; pp 297-325.

(7) Zhu, X.; Kawatkar, S.; Rao, Y.; Boons, G.-J. Practical Approach for the Sereoselective Introduction of $\beta$-Arabinofuranosides. J. Am. Chem. Soc. 2006, 128, 11948-11957.

(8) Ishiwata, A.; Akao, H.; Ito, Y. Stereoselective Synthesis of a Fragment of Mycobacterial Arabinan. Org. Lett. 2006, 8, 5525-5528.

(9) Gadikota, R. R.; Callam, C. S.; Wagner, T.; Del Fraino, B.; Lowary, T. L. 2,3-Anhydrosugars in Glycoside Bond Synthesis. Highly Stereoselective Syntheses of Oligosaccharides Containing $\alpha$ - and $\beta$ Arabinofuranosyl Linkages. J. Am. Chem. Soc. 2003, 125, 4155-4165.

(10) Oscarson, S.; Sehgelmeble, F. W. A Novel $\beta$-Directing Fructofuranosyl Donor Concept. Stereospecific Synthesis of Sucrose. J. Am. Chem. Soc. 2000, 122, 8869-8872.

(11) Bai, Y.; Lowary, T. L. 2,3-Anhydrosugars in Glycoside Bond Synthesis. Application to $\alpha$-D-Dalactofuranosides. J. Org. Chem. 2006, 71, 9658-9671.

(12) Tilve, M. J.; Gallo-Rodriguez, C. Glycosylation Studies on Conformationally Restricted 3,5-O-(di-tert-butylsilylene)-D-Galacto- 
furanosyl Trichloroacetimidate Donors for 1,2-cis- $\alpha$-D-Galactofuranosylation. Carbohydr. Res. 2011, 346, 2838-2848.

(13) Tilve, M. J.; Gallo-Rodriguez, C. Conformationally Restricted 3,5-O-(di-tert-butylsilylene)-D-Galactofuranosyl Thioglycoside Donor for 1,2-cis- $\alpha$-D-Galactofuranosylation. Carbohydr. Res. 2014, 397, 717.

(14) Imamura, A.; Lowary, T. L. $\beta$-Selective Arabinofuranosylation Using a 2,3-O-Xylylene-Protected Donor. Org. Lett. 2010, 12, 36863689.

(15) Larsen, C. H.; Ridgway, B. H.; Shaw, J. T.; Woerpel, K. A. A Stereoelectronic Model To Explain the Highly Stereoselective Reactions of Nucleophiles with Five-Membered-Ring Oxocarbenium Ions. J. Am. Chem. Soc. 1999, 121, 12208-12209.

(16) Turnbull, W. B.; Stalford, S. A. Methylthioxylose - A Jewel in the Mycobacterial Crown? Org. Biomol. Chem. 2012, 10, 5698-5706.

(17) Angala, S. K.; McNeil, M. R.; Shi, L.; Joe, M.; Pham, H.; Zuberogoitia, S.; Nigou, J.; Boot, C. M.; Lowary, T. L.; Gilleron, M.; Jackson, M. Biosynthesis of the Methylthioxylose Capping Motif of Lipoarabinomannan in Mycobacterium tuberculosis. ACS Chem. Biol. 2017, 12, 682-691.

(18) Joe, M.; Sun, D.; Taha, H.; Completo, G. C.; Croudace, J. E.; Lammas, D. A.; Besra, G. S.; Lowary, T. L. The 5-Deoxy-5methylthio-xylofuranose Residue in Mycobacterial Lipoarabinomannan. Absolute Stereochemistry, Linkage Position, Conformation, and Immunomodulatory Activity. J. Am. Chem. Soc. 2006, 128, 50595072.

(19) Zheng, R. B.; Jégouzo, S. A. F.; Joe, M.; Bai, Y.; Tran, H.-A.; Shen, K.; Saupe, J.; Xia, L.; Ahmed, M. F.; Liu, Y.-H.; Patil, P. S.; Tripathi, A.; Hung, S.-C.; Taylor, M. E.; Lowary, T. L.; Drickamer, K. Insights into Interactions of Mycobacteria with the Host Innate Immune System from a Novel Array of Synthetic Mycobacterial Glycans. ACS Chem. Biol. 2017, 12, 2990-3002.

(20) Stalford, S. A.; Kilner, C. A.; Leach, A. G.; Turnbull, W. B. Neighbouring Group Participation vs. Addition to Oxacarbenium Ions: Studies on the Synthesis of Mycobacterial Oligosaccharides. Org. Biomol. Chem. 2009, 7, 4842-4852.

(21) Mandal, P. K.; Chheda, P. R. Synthesis and Biotinylation of Oligosaccharide Fragments of Mannosylated and 5-Deoxy-5-methylthio-xylofuranosylated Lipoarabinomannan from Mycobacterium tuberculosis. Carbohydr. Res. 2015, 407, 104-110.

(22) Tilekar, J. N.; Lowary, T. L. An Efficient Route to Thioglycosides with the 2,3-anhydro-D-ribo-Stereochemistry. Carbohydr. Res. 2004, 339, 2895-2899.

(23) Imamura, A.; Lowary, T. Chemical Synthesis of Furanose Glycosides. Trends Glycosci. Glycotechnol. 2011, 23, 134-152.

(24) Kafle, A.; Liu, J.; Cui, L. Controlling the Stereoselectivity of Glycosylation via Solvent Effects. Can. J. Chem. 2016, 94, 894-901.

(25) Bohé, L.; Crich, D. A Propos of Glycosyl Cations and the Mechanism of Chemical Glycosylation. C. R. Chim. 2011, 14, 3-16.

(26) van Rijssel, E. R.; van Delft, P.; Lodder, G.; Overkleeft, H. S.; van der Marel, G. A.; Filippov, D. V.; Codée, J. D. C. Furanosyl Oxocarbenium Ion Stability and Stereoselectivity. Angew. Chem., Int. Ed. 2014, 53, 10381-10385.

(27) Mishra, A. K.; Krumbach, K.; Rittmann, D.; Appelmelk, B.; Pathak, V.; Pathak, A. K.; Nigou, J.; Geurtsen, J.; Eggeling, L.; Besra, G. S. Lipoarabinomannan Biosynthesis in Corynebacterineae: The Interplay of Two $\alpha(1 \rightarrow 2)$-Mannopyranosyltransferases $\mathrm{MptC}$ and MptD in Mannan Branching. Mol. Microbiol. 2011, 80, 1241-1259.

(28) Frisch, M. J.; Trucks, G. W.; Schlegel, H. B.; Scuseria, G. E.; Robb, M. A.; Cheeseman, J. R.; Scalmani, G.; Barone, V.; Mennucci, B.; Petersson, G. A.; Nakatsuji, H.; Caricato, M.; Li, X.; Hratchian, H. P.; Izmaylov, A. F.; Bloino, J.; Zheng, G.; Sonnenberg, J. L.; Hada, M.; Ehara, M.; Toyota, K.; Fukuda, R.; Hasegawa, J.; Ishida, M.; Nakajima, T.; Honda, Y.; Kitao, O.; Nakai, H.; Vreven, T.; Montgomery, J. A.; Peralta, J. E.; Ogliaro, F.; Bearpark, M.; Heyd, J. J.; Brothers, E.; Kudin, K. N.; Staroverov, V. N.; Keith, T.; Kobayashi, R.; Normand, J.; Raghavachari, K.; Rendell, A.; Burant, J. C.; Iyengar, S. S.; Tomasi, J.; Cossi, M.; Rega, N.; Millam, J. M.; Klene, M.; Knox, J. E.; Cross, J. B.; Bakken, V.; Adamo, C.; Jaramillo,
J.; Gomperts, R.; Stratmann, R. E.; Yazyev, O.; Austin, A. J.; Cammi, R.; Pomelli, C.; Ochterski, J. W.; Martin, R. L.; Morokuma, K.; Zakrzewski, V. G.; Voth, G. A.; Salvador, P.; Dannenberg, J. J.; Dapprich, S.; Daniels, A. D.; Farkas, O.; Foresman, J. B.; Ortiz, J. V.; Cioslowski, J.; Fox, D. J. Gauusian 09; Gaussian, Inc.: Wallingford, CT.

(29) Roothaan, C. C. J. New Developments in Molecular Orbital Theory. Rev. Mod. Phys. 1951, 23, 69-89.

(30) Krishnan, R.; Binkley, J. S.; Seeger, R.; Pople, J. A. SelfConsistent Molecular Orbital Methods. XX. A Basis Set for Correlated Wave Functions. J. Chem. Phys. 1980, 72, 650-654.

(31) McLean, A. D.; Chandler, G. S. Contracted Gaussian Basis Sets for Molecular Calculations. I. Second Row Atoms, $\mathrm{Z}=11-18$. J. Chem. Phys. 1980, 72, 5639-5648.

(32) Frisch, M. J.; Pople, J. A.; Binkley, J. S. Self-Consistent Molecular Orbital Methods 25. Supplementary Functions for Gaussian Basis Sets. J. Chem. Phys. 1984, 80, 3265-3269.

(33) Becke, A. D. Density Functional Thermochemistry. III. The Role of Exact Exchange. J. Chem. Phys. 1993, 98, 5648-5652.

(34) Rassolov, V. A.; Ratner, M. A.; Pople, J. A.; Redfern, P. C.; Curtiss, L. A. 6-31G* Basis Set for Third-Row Atoms. J. Comput. Chem. 2001, 22, 976-984. 